\title{
Approach in Theory of Nonlinear Evolution Equations: The Vakhnenko-Parkes Equation
}

\author{
V. O. Vakhnenko ${ }^{1}$ and E. J. Parkes ${ }^{2}$ \\ ${ }^{1}$ Institute of Geophysics, National Academy of Sciences of Ukraine, Kyiv 01054, Ukraine \\ ${ }^{2}$ Department of Mathematics \& Statistics, University of Strathclyde, Glasgow G1 1XH, UK \\ Correspondence should be addressed to V. O. Vakhnenko; vakhnenko@ukr.net
}

Received 13 September 2015; Accepted 12 November 2015

Academic Editor: Andrei D. Mironov

Copyright ( 2016 V. O. Vakhnenko and E. J. Parkes. This is an open access article distributed under the Creative Commons Attribution License, which permits unrestricted use, distribution, and reproduction in any medium, provided the original work is properly cited.

\begin{abstract}
A variety of methods for examining the properties and solutions of nonlinear evolution equations are explored by using the Vakhnenko equation (VE) as an example. The VE, which arises in modelling the propagation of high-frequency waves in a relaxing medium, has periodic and solitary traveling wave solutions some of which are loop-like in nature. The VE can be written in an alternative form, known as the Vakhnenko-Parkes equation (VPE), by a change of independent variables. The VPE has an N-soliton solution which is discussed in detail. Individual solitons are hump-like in nature whereas the corresponding solution to the VE comprises $N$ loop-like solitons. Aspects of the inverse scattering transform (IST) method, as applied originally to the KdV equation, are used to find one- and two-soliton solutions to the VPE even though the VPE's spectral equation is third-order and not secondorder. A Bäcklund transformation for the VPE is used to construct conservation laws. The standard IST method for third-order spectral problems is used to investigate solutions corresponding to bound states of the spectrum and to a continuous spectrum. This leads to $N$-soliton solutions and $M$-mode periodic solutions, respectively. Interactions between these types of solutions are investigated.
\end{abstract}

\section{Introduction}

The physical phenomena and processes that take place in nature generally have complicated nonlinear features. This leads to nonlinear mathematical models for the real processes. There is much interest in the practical issues involved, as well as the development of methods to investigate the associated nonlinear mathematical problems including nonlinear wave propagation. An early example of the latter was the development of the inverse scattering method for the Korteweg-de Vries (KdV) equation [1] and the subsequent interest in soliton theory. Now soliton theory is applied in many branches of science.

The modern physicist should be aware of aspects of nonlinear wave theory developed over the past few years. This paper focuses on the connection between a variety of different approaches and methods. The application of the theory of nonlinear evolution equations to study a new equation is always an important step. Based on our experience of the study of the Vakhnenko equation (VE), we acquaint the reader with a series of methods and approaches which may be applied to certain nonlinear equations. Thus we outline a way in which an uninitiated reader could investigate a new nonlinear equation.

\section{A Model for High-Frequency Waves in a Relaxing Medium}

Starting from a general idea of relaxing phenomena in real media via a hydrodynamic approach, we will derive a nonlinear evolution equation for describing high-frequency waves. To develop physical models for wave propagation through media with complicated inner kinetics, notions based on the relaxational nature of a phenomenon are regarded to 
be promising. From the nonequilibrium thermodynamics standpoint, models of a relaxing medium are more general than equilibrium models. Thermodynamic equilibrium is disturbed owing to the propagation of fast perturbations. There are processes of the interaction that tend to return the equilibrium. The parameters characterizing this interaction are referred to as the inner variables unlike the macroparameters such as the pressure $p$, mass velocity $u$, and density $\rho$. In essence, the change of macroparameters caused by the changes of inner parameters is a relaxation process.

We restrict our attention to barotropic media. An equilibrium state equation of a barotropic medium is a oneparameter equation. As a result of relaxation, an additional variable $\xi$ (the inner parameter) appears in the state equation

$$
p=p(\rho, \xi)
$$

and defines the completeness of the relaxation process. There are two limiting cases with corresponding sound velocities:

(i) Lack of relaxation (inner interaction processes are frozen) for which $\xi=1$ :

$$
\begin{aligned}
p & =p(\rho, 1) \equiv p_{f}(\rho), \\
c_{f}^{2} & =\frac{d p_{f}}{d \rho} .
\end{aligned}
$$

(ii) Relaxation which is complete (there is local thermodynamic equilibrium) for which $\xi=0$ :

$$
\begin{aligned}
p & =p(\rho, 0) \equiv p_{e}(\rho), \\
c_{e}^{2} & =\frac{d p_{e}}{d \rho} .
\end{aligned}
$$

Slow and fast processes are compared by means of the relaxation time $\tau_{p}$.

To analyze the wave motion, we use the following hydrodynamic equations in Lagrangian coordinates:

$$
\begin{aligned}
& \frac{\partial V}{\partial t}-\frac{1}{\rho_{0}} \frac{\partial u}{\partial x}=0 \\
& \frac{\partial u}{\partial t}+\frac{1}{\rho_{0}} \frac{\partial p}{\partial x}=0 .
\end{aligned}
$$

The following dynamic state equation is applied to account for the relaxation effects:

$$
\tau_{p}\left(\frac{d p}{d t}-c_{f}^{2} \frac{d \rho}{d t}\right)+\left(p-p_{e}\right)=0 .
$$

Here $V \equiv \rho^{-1}$ is the specific volume, and $x$ is the Lagrangian space coordinate. Clearly, for the fast processes $\left(\omega \tau_{p} \gg 1\right)$, we have relation (2), and for the slow ones $\left(\omega \tau_{p} \ll 1\right)$ we have (3).

The closed system of equations consists of two motion equations (4) and the dynamic state equation (5). The motion equations (4) are written in Lagrangian coordinates since the state equation (5) is related to the element of mass of the medium.

The substantiation of (5) within the framework of the thermodynamics of irreversible processes has been given in $[2,3]$. We note that the mechanisms of the exchange processes are not defined concretely when deriving the dynamic state equation (5). In this equation the thermodynamic and kinetic parameters appear only as sound velocities $c_{e}, c_{f}$ and relaxation time $\tau_{p}$. These are very common characteristics and they can be found experimentally. Hence it is not necessary to know the inner exchange mechanism in detail.

Let us consider a small nonlinear perturbation $p^{\prime}<$ $p_{0}$. Combining the relationships (4) and (5) we obtain the following nonlinear evolution equation in one unknown $p$ (the dash in $p^{\prime}$ is omitted) [4-6]:

$$
\begin{gathered}
\tau_{p} \frac{\partial}{\partial t}\left(\frac{\partial^{2} p}{\partial x^{2}}-c_{f}^{-2} \frac{\partial^{2} p}{\partial t^{2}}+\alpha_{f} \frac{\partial^{2} p^{2}}{\partial t^{2}}\right) \\
+\left(\frac{\partial^{2} p}{\partial x^{2}}-c_{e}^{-2} \frac{\partial^{2} p}{\partial t^{2}}+\alpha_{e} \frac{\partial^{2} p^{2}}{\partial t^{2}}\right)=0 \\
\alpha_{e}=\left.\frac{1}{2 V_{0}^{2}} \frac{d^{2} V_{e}}{d p^{2}}\right|_{p=p_{0}}, \alpha_{f}=\left.\frac{1}{2 V_{0}^{2}} \frac{d^{2} V_{f}}{d p^{2}}\right|_{p=p_{0}} .
\end{gathered}
$$

A similar equation has been obtained by Clarke [2], but without nonlinear terms. In [4] it is shown by the multiscale method [7] that for low-frequency perturbations $\left(\tau_{p} \omega \ll\right.$ 1) (6) is reduced to the Korteweg-de Vries-Burgers (KdVB) equation:

$$
\begin{aligned}
& \frac{\partial p}{\partial t}+c_{e} \frac{\partial p}{\partial x}+\alpha_{e} c_{e}^{3} p \frac{\partial p}{\partial x}-\beta_{e} \frac{\partial^{2} p}{\partial x^{2}}+\gamma_{e} \frac{\partial^{3} p}{\partial x^{3}}=0, \\
& \beta_{e}=\frac{c_{e}^{2} \tau_{p}}{2 c_{f}^{2}}\left(c_{f}^{2}-c_{e}^{2}\right), \gamma_{e}=\frac{c_{e}^{3} \tau_{p}^{2}}{8 c_{f}^{4}}\left(c_{f}^{2}-c_{e}^{2}\right)\left(c_{f}^{2}-5 c_{e}^{2}\right),
\end{aligned}
$$

while for high-frequency waves $\left(\tau_{p} \omega \gg 1\right)$ we have obtained the following equation:

$$
\begin{aligned}
\frac{\partial^{2} p}{\partial x^{2}}-c_{f}^{-2} \frac{\partial^{2} p}{\partial t^{2}}+\alpha_{f} c_{f}^{2} \frac{\partial^{2} p^{2}}{\partial x^{2}}+\beta_{f} \frac{\partial p}{\partial x}+\gamma_{f} p & =0 \\
\beta_{f} & =\frac{c_{f}^{2}-c_{e}^{2}}{\tau_{p} c_{e}^{2} c_{f}}, \gamma_{f}=\frac{c_{f}^{4}-c_{e}^{4}}{2 \tau_{p}^{2} c_{e}^{4} c_{f}^{2}} .
\end{aligned}
$$

Equation (7) is the well-known KdVB equation. It is encountered in many areas of physics to describe nonlinear wave processes [8]. In [9] it was shown how hydrodynamic equations reduce to either the $\mathrm{KdV}$ or Burgers equation according to the choices for the state equation and the generalized force when analyzing gasdynamical waves, waves in shallow water [9], hydrodynamic waves in cold plasma [10], and ion-acoustic waves in cold plasma [11].

As is known, the investigation of the $\mathrm{KdV}$ equation $\left(\beta_{e}=\right.$ 0 ) in conjunction with the nonlinear Schrödinger (NLS) and sine-Gordon equations gives rise to the theory of solitons [1, $8,9,12-18]$. 
We focus our main attention on (8). It has a dissipative term $\beta_{f} \partial p / \partial x$ and a dispersive term $\gamma_{f} p$. Without the nonlinear and dissipative terms, we have a linear Klein-Gordon equation. At the time we were carrying out our research, it turned out that (8) had not been investigated much. It is likely that this is connected with the fact, noted by Whitham [19], that high-frequency perturbations attenuate very quickly. However in Whitham's monograph, the evolution equation without nonlinear and dispersive terms was considered.

Note the fact that the dispersion relations $\omega=\omega(k)$ for the linearized versions of (7) and (8) are restricted to finite power series in $k$ and in $k^{-1}$, respectively:

$$
\begin{aligned}
\omega & =c_{e} k+i \beta_{e} k^{2}-\gamma_{e} k^{3}, \quad \tau_{p} \omega \ll 1, \\
\omega^{2} & =c_{f}^{2} k^{2}\left(1+i \beta_{f} k^{-1}-\gamma_{f} k^{-2}\right), \quad \tau_{p} \omega \gg 1 .
\end{aligned}
$$

Let us write down (8) in dimensionless form. In the moving coordinates system with velocity $c_{f}$, after factorization the equation has the form in the dimensionless variables $\tilde{x}=$ $\sqrt{\gamma_{f} / 2}\left(x-c_{f} t\right), \tilde{t}=\sqrt{\gamma_{f} / 2} c_{f} t, \tilde{u}=\alpha_{f} c_{f}^{2} p$ (tilde over variables $\tilde{x}, \tilde{t}, \tilde{u}$ is omitted):

$$
\frac{\partial}{\partial x}\left(\frac{\partial}{\partial t}+u \frac{\partial}{\partial x}\right) u+\alpha \frac{\partial u}{\partial x}+u=0 .
$$

The constant $\alpha=\beta_{f} / \sqrt{2 \gamma_{f}}$ is always positive. Equation (10) without the dissipative term has the form of the nonlinear equation [20, 21]:

$$
\frac{\partial}{\partial x}\left(\frac{\partial}{\partial t}+u \frac{\partial}{\partial x}\right) u+u=0 .
$$

Historically, (11) has been called the Vakhnenko equation (VE) and we will follow this name.

It is interesting to note that (11) follows as a particular limit of the following generalized Korteweg-de Vries equation:

$$
\frac{\partial}{\partial x}\left(\frac{\partial u}{\partial t}+u \frac{\partial u}{\partial x}-\beta \frac{\partial^{3} u}{\partial x^{3}}\right)=\gamma u
$$

derived by Ostrovsky [22] to model small-amplitude long waves in a rotating fluid ( $\gamma u$ is induced by the Coriolis force) of finite depth. Subsequently, (11) was known by different names in the literature, such as the Ostrovsky-Hunter equation, the short-wave equation, the reduced Ostrovsky equation, and the Ostrovsky-Vakhnenko equation depending on the physical context in which it is studied.

The consideration here of (11) has interest not only from the viewpoint of the investigation of the propagation of highfrequency perturbations, but also more specifically from the viewpoint of the study of methods and approaches that may be applied in the theory of nonlinear evolution equations.

\section{Loop-Like Stationary Solutions and Their Stability}

By investigating (11), we will trace a way in which an uninitiated reader could investigate a new nonlinear equation. As a first step for a new equation, it is necessary to consider the linear analogue and its dispersion relation (these steps for (7) and (8) are described already in Section 2). The next step is, where possible, to link the equation with a known nonlinear equation.

3.1. The Connection of the VE with the Whitham Equation. Now we show how an evolution equation with hydrodynamic nonlinearity can be rewritten in the form of the Whitham equation. The general form of the Whitham equation is as follows [19]:

$$
\frac{\partial u}{\partial t}+u \frac{\partial u}{\partial x}+\int_{-\infty}^{\infty} K(x-s) \frac{\partial u}{\partial s} d s=0 .
$$

On one hand, (13) has the nonlinearity of hydrodynamic type; on the other hand, it is known (see Section 13.14 in [19]) that the kernel $K(x)$ can be selected to give the dispersion required. Indeed, the dispersion relation $c(k)=\omega(k) / k$ and the kernel $K(x)$ are connected by means of the Fourier transformation:

$$
\begin{aligned}
c(k) & =F[K(x)], \\
K(x) & =F^{-1}[c(k)] .
\end{aligned}
$$

Consequently, for the dispersion relation $\omega=-1 / k$ corresponding to the linearized version of (11), the kernel is as follows:

$$
K(x)=F^{-1}\left[-\frac{1}{k^{2}}\right]=\frac{1}{2}|x| .
$$

Thus, the VE (11) is related to the particular Whitham equation [19]:

$$
\frac{\partial u}{\partial t}+u \frac{\partial u}{\partial x}+\frac{1}{2} \int_{-\infty}^{\infty}|x-s| \frac{\partial u}{\partial s} d s=0 .
$$

Since we can reduce the VE to the Whitham equation, we can assert that the VE shares interesting properties with the Whitham equation; in particular, it describes solitary wave-type formations, has periodic solutions, and explains the existence of the limiting amplitude [19]. An important property is the presence of conservation laws for waves decreasing rapidly at infinity; namely,

$$
\begin{aligned}
\frac{d}{d t} \int_{-\infty}^{\infty} u d x & =0, \\
\frac{d}{d t} \int_{-\infty}^{\infty} u^{2} d x & =0 \\
\frac{d}{d t} \int_{-\infty}^{\infty}\left(\frac{1}{3} u^{3}+\widehat{K} u\right) d x & =0
\end{aligned}
$$

where by definition $\widehat{K} u=\int_{-\infty}^{\infty} K(x-s) u(s, t) d s$.

For (10) the kernel is $K(x)=(1 / 2)[\alpha(2 \Theta(x)-1)+|x|]$, where $\Theta(x)$ is the Heaviside function. Hence, (10) can be written down as

$$
\frac{\partial u}{\partial t}+u \frac{\partial u}{\partial x}+\alpha u+\frac{1}{2} \int_{-\infty}^{\infty}|x-s| \frac{\partial u}{\partial s} d s=0 .
$$

There is no derivative in the dissipative term $\alpha u$ of (18). 
3.2. The Traveling Wave Solutions. An important step in the investigation of nonlinear evolution equations is to find traveling wave solutions. These are solutions which are stationary with respect to a moving frame of reference. In this case, the evolution equation (a partial differential equation) becomes an ordinary differential equation (ODE) which is considerably easier to solve.

For the VE (11) it is convenient to introduce a new dependent variable $z$ and new independent variables $\eta$ and $\tau$ defined by

$$
\begin{aligned}
& z=\frac{(u-v)}{|v|}, \\
& \eta=\frac{(x-v t)}{|v|^{1 / 2}}, \\
& \tau=t|v|^{1 / 2},
\end{aligned}
$$

where $v$ is a nonzero constant [21]. Then the VE becomes

$$
z_{\eta \tau}+\left(z z_{\eta}\right)_{\eta}+z+c=0
$$

where $c= \pm 1$ corresponding to $v \gtrless 0$. We now seek stationary solutions of (20) for which $z$ is a function of $\eta$ only so that $z_{\tau}=0$ and $z$ satisfies the ODE

$$
\left(z z_{\eta}\right)_{\eta}+z+c=0
$$

After one integration (21) gives

$$
\begin{aligned}
& \frac{1}{2}\left(z z_{\eta}\right)^{2}=f(z), \\
& f(z)=-\frac{1}{3} z^{3}-\frac{1}{2} c z^{2}+\frac{1}{6} A=-\frac{1}{3}\left(z-z_{1}\right)\left(z-z_{2}\right)\left(z-z_{3}\right) .
\end{aligned}
$$

$A$ is a constant and for periodic solutions $z_{1}, z_{2}$, and $z_{3}$ are real constants such that $z_{1} \leq z_{2} \leq z_{3}$. On using results 236.00 and 236.01 of [23], we may integrate (22) to obtain

$$
\begin{aligned}
\eta & =\frac{\sqrt{6} z_{1}}{\sqrt{z_{3}-z_{1}}} F(\varphi, m)+\sqrt{6\left(z_{3}-z_{1}\right)} E(\varphi, m), \\
\sin \varphi & =\frac{z_{3}-z}{z_{3}-z_{2}} \\
m & =\frac{z_{3}-z_{2}}{z_{3}-z_{1}} .
\end{aligned}
$$

$F(\varphi, m)$ and $E(\varphi, m)$ are incomplete elliptic integrals of the first and second kind, respectively. We have chosen the constant of integration in (23) to be zero so that $z=z_{3}$ at $\eta=0$. The relations (23) give the required solution in parametric form, with $z$ and $\eta$ as functions of the parameter $\varphi$.

An alternative route to the solution is to follow the procedure described in [24]. We introduce a new independent variable $\zeta$ defined by

$$
\frac{d \eta}{d \zeta}=z
$$

so that (22) becomes

$$
\frac{1}{2} z_{\zeta}^{2}=f(z)
$$

By means of result 236.00 of [23], (26) may be integrated to give $p \zeta=F(\varphi, m)$, where $p^{2}=\left(z_{3}-z_{1}\right) / 6$. Thus, on noting that $\sin \varphi=\operatorname{sn}(p \zeta \mid w)$, where sn is a Jacobian elliptic function, we have

$$
z=z_{3}-\left(z_{3}-z_{2}\right) \operatorname{sn}^{2}(p \zeta \mid w) .
$$

With result 310.02 of [23], (25) and (27) give

$$
\eta=z_{1} \zeta+\sqrt{6\left(z_{3}-z_{1}\right)} E(p \zeta)
$$

where $E(p \zeta):=E(\operatorname{am} p \zeta, m)$. Relations (27) and (28) are equivalent to (24) and (23), respectively, and give the solution in parametric form with $z$ and $\eta$ in terms of the parameter $\zeta$.

We define the wavelength $\lambda$ of the solution as the amount by which $\eta$ increases when $\varphi$ increases by $2 \pi$; from (23) we obtain

$$
\lambda=\frac{2 \sqrt{6}}{\sqrt{z_{3}-z_{1}}}\left[z_{1} K(m)+\left(z_{3}-z_{1}\right) E(m)\right],
$$

where $K(m)$ and $E(m)$ are complete elliptic integrals of the first and second kind, respectively.

For $c=1$ (i.e., $v>0$ ), there are periodic solutions for $0<A<1$ with $\lambda<0, z_{2} \in(-1,0)$, and $z_{3} \in(0,0.5)$; an example of such a periodic wave is illustrated by curve 2 in Figure 1. $A=1$ gives the solitary wave limit

$$
\begin{aligned}
& u=\frac{3}{2} v \operatorname{sech}^{2}\left(\frac{\zeta}{2}\right), \\
& \eta=-\zeta+3 \tanh \left(\frac{\zeta}{2}\right)
\end{aligned}
$$

as illustrated by curve 1 in Figure 1 . The periodic waves and the solitary wave have a loop-like structure as illustrated in Figure 1. For $c=-1$ (i.e., $v<0$ ), there are periodic waves for $-1<A<0$ with $\lambda>0, z_{2} \in(0,1)$, and $z_{3} \in(1,1.5)$; an example of such a periodic wave is illustrated by curve 2 in Figure 2. When $A=0$ and $\lambda=6$ the periodic wave solution simplifies to

$$
\frac{u(\eta)}{|v|}=-\frac{1}{6} \eta^{2}+\frac{1}{2}, \quad-3 \leq \eta \leq 3, u(\eta+6)=u(\eta) .
$$

This is shown by curve 1 in Figure 2 . For $A \simeq-1$ the solution has a sinusoidal form (curve 3 in Figure 2). Note that there are no solitary wave solutions.

A remarkable feature of (11) is that it has a solitary wave (30) which has loop-like form; that is, it is a multivalued function (see Figure 1). Whilst loop solitary waves (30) are rather intriguing, it is the solution to the initial value problem that is of more interest in a physical context. An important question is the stability of the loop-like solutions. Although the analysis of stability does not link with the theory of solitons directly, however, the method applied in 


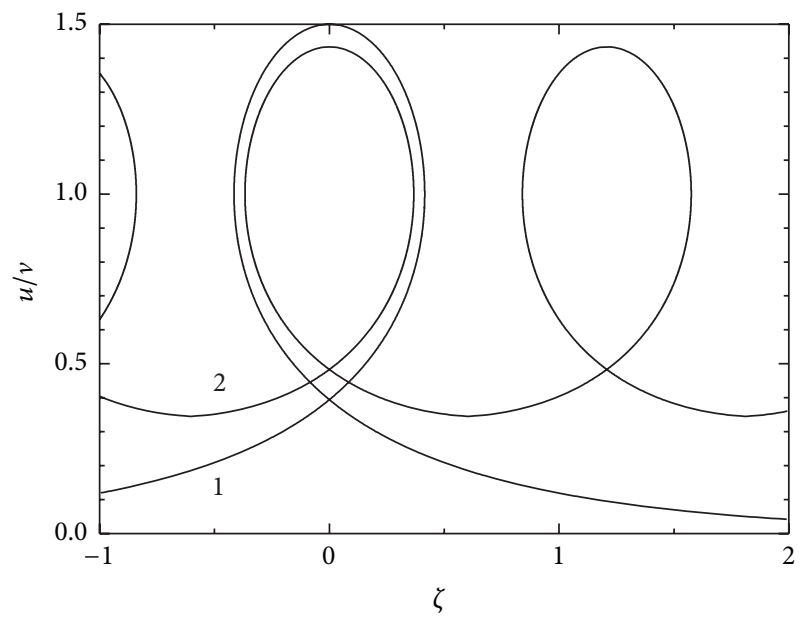

FIGURE 1: Traveling wave solutions with $v>0$.

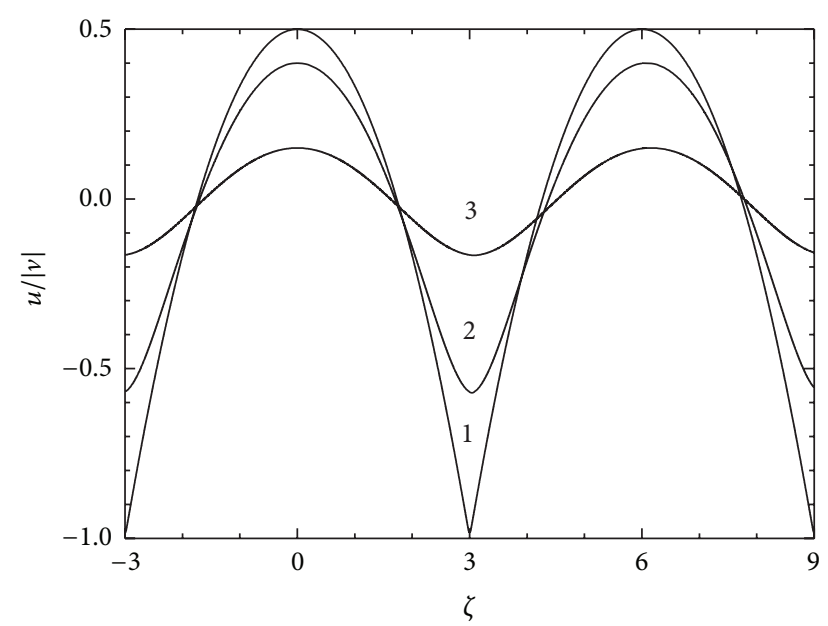

FIgURE 2: Traveling wave solutions with $v<0$.

Section 3.3 is instructive, since it is successful in a nonlinear approximation.

We note that the notion of a "soliton" will be defined later. We will prove (see Section 5.4) that the solitary wave (30) is, in fact, a soliton. Now we point only out that the soliton is a local traveling wave pulse with remarkable stability and particlelike properties.

3.3. Stability and Interpretation of the Loop-Like Solutions. From a physical viewpoint, the stability or otherwise of solutions is essential for their interpretation. Some methods for the investigation of the stability of nonlinear waves were discussed by Infeld and Rowlands in Chapter 8 of [25] and references therein. One such method is the so-called $k$ expansion method. It is restricted to long wavelength perturbations of small amplitude. It has been applied successfully to a variety of generic nonlinear evolution equations (see [26], e.g.) and specific physical systems (see [27], e.g.). A particularly informative description of the method is given in [28] in the context of the Zakharov-Kuznetsov equation. Some criticism was levelled at the work in [28] by Das et al.
[29]; however, after a detailed reinvestigation of the problem, Das et al. [29] vindicated the method used in [28].

The $k$-expansion method was applied to the VE (11) in [21] and is outlined as follows. We assume a perturbed solution of (21) in the form

$$
z=z_{0}(\eta)+\{\delta z(\eta) \exp [i(k \eta-\omega \tau)]+c c\}
$$

where $z_{0}$ is the periodic solution given by (27) and (28), $\delta z(\eta)$ is a complex function with period $\lambda$ given by (29), $k$ is a real constant, $\omega$ is a constant (possibly complex), and cc denotes complex conjugate. Substitution of (32) into (20) and linearization with respect to $\delta z$ yield

$$
\mathscr{L} \delta z=f
$$

where the linear operator $\mathscr{L}$ and $f$ are given by

$$
\begin{aligned}
\mathscr{L} \delta z & :=\left(z_{0} \delta z\right)_{\eta \eta}+\delta z, \\
f & :=\left(-\omega k+k^{2} z_{0}\right) \delta z+i\left[\omega \delta z_{\eta}-2 k\left(z_{0} \delta z\right)_{\eta}\right],
\end{aligned}
$$

respectively. As (21) implies that $\mathscr{L} z_{0 \eta}=0$, we may deduce that, for (33) to have periodic solutions, the condition

$$
\left\langle z_{0} z_{0 \eta} f\right\rangle=0
$$

must be satisfied, where $\langle\cdot\rangle$ denotes an integration over the wavelength $\lambda$.

Formally, the solution of (33) is

$$
\delta z=z_{0 \eta} \vartheta
$$

where

$$
\vartheta_{\eta}=\frac{\left(D+\int z_{0} z_{0 \eta} f d \eta\right)}{\left(z_{0} z_{0 \eta}\right)^{2}}
$$

and $D$ is a constant determined from

$$
\left\langle\frac{\left(D+\int z_{0} z_{0 \eta} f d \eta\right)}{\left(z_{0} z_{0 \eta}\right)^{2}}\right\rangle=0 .
$$

As $\delta z$ appears on the right-hand side of (36) via $f$, we solve (36) iteratively by assuming that $k$ is small in comparison with $2 \pi / \lambda$ (so that the perturbations in (32) have long wavelength) and introduce the expansions

$$
\begin{gathered}
\delta z=\delta z_{0}+k \delta z_{1}+\cdots, \\
\omega=k \omega_{1}+k^{2} \omega_{2}+\cdots,
\end{gathered}
$$

so that

$$
\begin{gathered}
f=k f_{1}+k^{2} f_{2}+\cdots, \\
\vartheta=\vartheta_{0}+k \vartheta_{1}+\cdots, \\
D=D_{0}+k D_{1}+\cdots .
\end{gathered}
$$


At zero order in $k$, condition (35) is satisfied identically, (38) gives $D_{0}=0$, and then, from (37), $\vartheta$ is constant. Hence, from (36), we may take $\delta z_{0}=z_{0 \eta}$. At first order, condition (35) is again satisfied identically. It is straightforward (see [21]) to find $D_{1}$ from (38) and $\vartheta_{1 \eta}$ from (37); use of these expressions in (35) at second order leads to the desired nonlinear dispersion relation for the perturbations in the form

$$
r_{0}+r_{1} \omega_{1}+r_{2} \omega_{1}^{2}=0
$$

The coefficients $r_{0}, r_{1}$, and $r_{2}$ depend on $z_{1}, z_{2}$, and $z_{3}$ as defined in (22). It turns out that the dispersion relation (41) has real roots for $\omega_{1}$ for both the families of solutions (corresponding to $c=1$ and $c=-1$, resp.) derived in Section 3.2. Consequently, it is predicted that both families of solutions are stable to long wavelength perturbations. For the loop-like solutions, the existence of singular points at which the derivatives tend to infinity casts some doubts on the validity of the method. However, in [21] it is argued that as the method depends on the average behaviour over a wavelength, the method is indeed valid.

The ambiguous structure of the loop-like solutions is similar to the loop soliton solution to an equation that models a stretched rope [30]. Loop-like solitons on a vortex filament were investigated by Hasimoto [31] and Lamb [32]. From the mathematical point of view, an ambiguous solution does not present difficulties whereas the physical interpretation of ambiguity always presents some difficulties. In this connection the problem of ambiguous solutions is regarded as important. The problem consists in whether the ambiguity has a physical nature or is related to the incompleteness of the mathematical model, in particular to the lack of dissipation.

We will consider the problem related to the singular points when dissipation takes place. At these points the dissipative term $\alpha(\partial u / \partial x)$ tends to infinity. The question arises: are there solutions of (18) in a loop-like form? The fact that the dissipation is likely to destroy the loop-like solutions can be associated with the following well-known fact [8]. For the simplest nonlinear equation without dispersion and without dissipation, namely,

$$
\frac{\partial u}{\partial t}+u \frac{\partial u}{\partial x}=0
$$

any initial smooth solution with boundary conditions

$$
\begin{aligned}
& \left.u\right|_{x \rightarrow+\infty}=0, \\
& \left.u\right|_{x \rightarrow-\infty}=u_{0}=\text { const. }>0
\end{aligned}
$$

becomes ambiguous in the final analysis. When dissipation is considered, we have the Burgers equation [33]:

$$
\frac{\partial u}{\partial t}+u \frac{\partial u}{\partial x}+\mu \frac{\partial^{2} u}{\partial x^{2}}=0 .
$$

The dissipative terms in this equation and in (7) for low frequency are coincident. The inclusion of the dissipative term transforms the solutions so that they cannot be ambiguous as a result of evolution. The wave parameters are always unambiguous. What happens in our case for high frequency when the dissipative term has the form $\alpha u$ (see (18))? Will the inclusion of dissipation give rise to unambiguous solutions?

By direct integration of (10) (written in terms of variables (19)) within the neighborhood of singular points $z=0$, where $z_{\eta} \rightarrow \pm \infty$ and $z_{\tau} \ll z_{\eta}$, it can be derived (see [4]) that the dissipative term, with dissipation parameter less than some limit value $\alpha^{*}$, does not destroy the loop-like solutions. Now we give a physical interpretation to ambiguous solutions.

Since the solution to the VE has a parametric form (23), (24) or (27), (28), there is a space of variables in which the solution is a single-valued function. Hence, we can solve the problem of the ambiguous solution. A number of states with their thermodynamic parameters can occupy one microvolume. It is assumed that the interaction between the separated states occupying one microvolume can be neglected in comparison with the interaction between the particles of one thermodynamic state. Even if we take into account the interaction between the separated states in accordance with the dynamic state equation (5) then, for high frequencies, a dissipative term arises which is similar to the corresponding term in (8), but with the other relaxation time. In this sense the separated terms are distributed in space, but describing the wave process we consider them as interpenetrable. A similar situation, when several components with different hydrodynamic parameters occupy one microvolume, has been assumed in mixture theory (see, e.g., $[34,35])$. Such a fundamental assumption in the theory of mixtures is physically impossible (see [34, page 7]), but it is appropriate in the sense that separated components are multivelocity interpenetrable continua.

Consequently, the following three observations show that, in the framework of the approach considered here, there are multivalued solutions when we model high-frequency wave processes: (1) All parts of loop-like solution are stable to perturbations. (2) Dissipation does not destroy the loop-like solutions. (3) The investigation regarding the interaction of the solitons has shown that it is necessary to take into account the whole ambiguous solution, not just the separate parts.

\section{The Vakhnenko-Parkes Equation}

The multivalued solutions obtained in Section 3.3 obviously mean that the study of the VE (11) in the original coordinates $(x, t)$ leads to certain difficulties. These difficulties can be avoided by writing down the VE in new independent coordinates. We have succeeded in finding these coordinates. Historically, working separately, we (Vyacheslav Vakhnenko in Ukraine and John Parkes in UK) independently suggested such independent coordinates in which the solutions become one-valued functions. It is instructive to present the two derivations here. In one derivation a physical approach, namely, a transformation between Euler and Lagrange coordinates, was used whereas in the other derivation a pure mathematical approach was used.

Let us define new independent variables $(X, T)$ by the transformation

$$
\varphi d T=d x-u d t, \quad X=t .
$$


The function $\varphi$ is to be obtained. It is important that the functions $x=\theta(X, T)$ and $u=U(X, T)$ turn out to be single-valued. In terms of the coordinates $(X, T)$ the solution of the VE (11) is given by single-valued parametric relations. The transformation into these coordinates is the key point in solving the problem of the interaction of solitons as well as explaining the multiple-valued solutions [4]. Transformation (45) is similar to the transformation between Eulerian coordinates $(x, t)$ and Lagrangian coordinates $(X, T)$. We require that $T=x$ if there is no perturbation, that is, if $u(x, t)=0$. Hence $\varphi=1$ when $u(x, t)=0$.

The function $\varphi$ is the additional dependent variable in the equation system (47) and (48) to which we reduce the original equation (11). We note that the transformation inverse to (45) is

$$
d x=\varphi d T+U d X, \quad t=X, U(X, T) \equiv u(x, t) .
$$

Then, by taking into account the condition that $d x$ is an exact differential, we obtain

$$
\frac{\partial \varphi}{\partial X}=\frac{\partial U}{\partial T}
$$

This equation, together with (11) rewritten in terms of $\varphi(X, T)$ and $U(X, T)$, namely,

$$
\frac{\partial^{2} \varphi}{\partial X^{2}}+U \varphi=0
$$

is the main system of equations. The equation system (47) and (48) can be reduced to a nonlinear equation in one unknown $W$ defined by

$$
W_{X}=U
$$

We study solutions $U$ that vanish as $|X| \rightarrow \infty$ or, equivalently, solutions for which $W$ tends to a constant as $|X| \rightarrow \infty$. From (47) and (49) and the requirement that $\varphi \rightarrow 1$ as $|X| \rightarrow \infty$ we have $\varphi=1+W_{T}$; then, by eliminating $\varphi$ from (48) we arrive at the transformed form of the VE (11)

$$
W_{X X T}+\left(1+W_{T}\right) W_{X}=0
$$

or, in equivalent form,

$$
U U_{X X T}-U_{X} U_{X T}+U^{2} U_{T}=0 .
$$

Furthermore it follows from (46) that the original independent space coordinate $x$ is given by

$$
x=\theta(X, T)=x_{0}+T+W,
$$

where $x_{0}$ is an arbitrary constant. Since the functions $\theta(X, T)$ and $U(X, T)$ are single-valued, the problem of multivalued solutions has been resolved from the mathematical point of view.

Alternatively, in a pure mathematical approach, we introduce new independent variables $X, T$ defined by

$$
x=\theta(X, T)=T+\int_{-\infty}^{X} U\left(X^{\prime}, T\right) d X^{\prime}+x_{0}, \quad t=X,
$$

where $u(x, t)=U(X, T)$ and $x_{0}$ is a constant. From (53) it follows that

$$
\begin{aligned}
\frac{\partial}{\partial X} & =\frac{\partial}{\partial t}+u \frac{\partial}{\partial x} \\
\frac{\partial}{\partial T} & =\phi \frac{\partial}{\partial x}
\end{aligned}
$$

$$
\text { with } \phi(X, T)=1+\int_{-\infty}^{X} U_{T} d X^{\prime}
$$

so that

$$
\phi_{X}=U_{T}
$$

From (11) and (54) we obtain

$$
U_{X T}+\phi U=0
$$

By eliminating $\phi$ between (55) and (56) we obtain (51) or, on introducing $W,(50)$.

The transformation, as has already been pointed out, was obtained by us independently of each other; nevertheless, we published the result together [36-38]. Following [39-42], hereafter (50) (or in alternative form (51)) is referred to as the Vakhnenko-Parkes equation (VPE).

For example, we will rewrite the solutions (23) and (24) for (11) in the transformed coordinates $(X, T)$, that is, find the traveling wave solutions for (11) in new coordinates. Differentiating the relationship (23) with respect to $X$, we take

$$
\begin{aligned}
\pm \sqrt{\frac{2}{3} \frac{\partial \eta}{\partial X}} & = \pm \sqrt{\frac{2}{3}}\left(-v+\frac{\partial x}{\partial X}\right) \\
& = \pm \sqrt{\frac{2}{3}}\left(-v+W_{X}(X, T)\right)= \pm \sqrt{\frac{2}{3}} z \\
& =\frac{z}{\sqrt{\left(z-a_{1}\right)\left(z-a_{2}\right)\left(a_{3}-z\right)}} \frac{\partial z}{\partial X}
\end{aligned}
$$

or

$$
\pm \sqrt{\frac{2}{3}}=\frac{1}{\sqrt{\left(z-a_{1}\right)\left(z-a_{2}\right)\left(a_{3}-z\right)}} \frac{\partial z}{\partial X} .
$$

Then after integration, we obtain

$$
\begin{aligned}
\pm \sqrt{\frac{2}{3}} X & =\int_{z}^{a_{3}} \frac{d z}{\sqrt{\left(z-a_{1}\right)\left(z-a_{2}\right)\left(a_{3}-z\right)}} \\
& =\frac{2}{\sqrt{a_{3}-a_{1}}} F(\varphi, k) .
\end{aligned}
$$

Together with $z=U(X, T)+v$ this relationship (59) determines the desired dependence $U(X, T)$ in parametrical form. Thus, we have the solution for (11) in new coordinates $(X, T)$. 


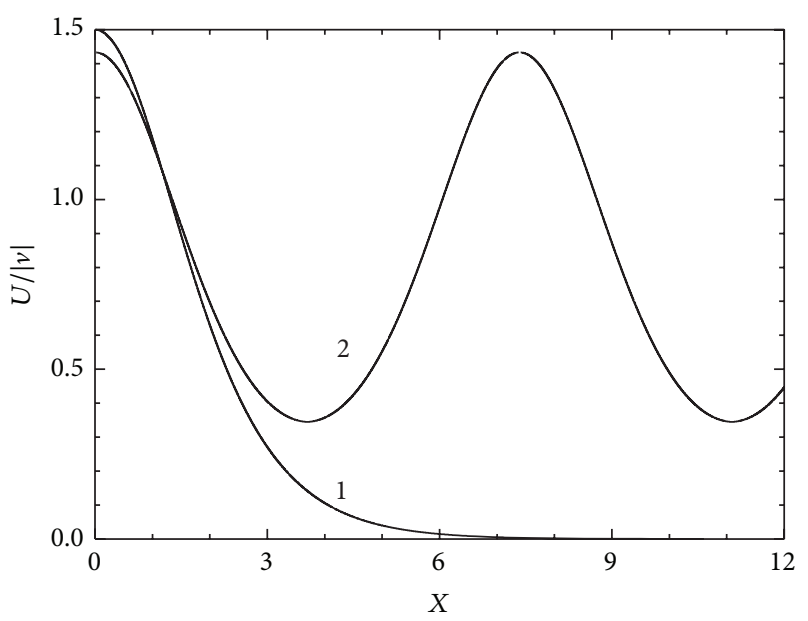

FIgURE 3: Traveling wave solutions with $v>0$ in coordinates $(X, T)$.

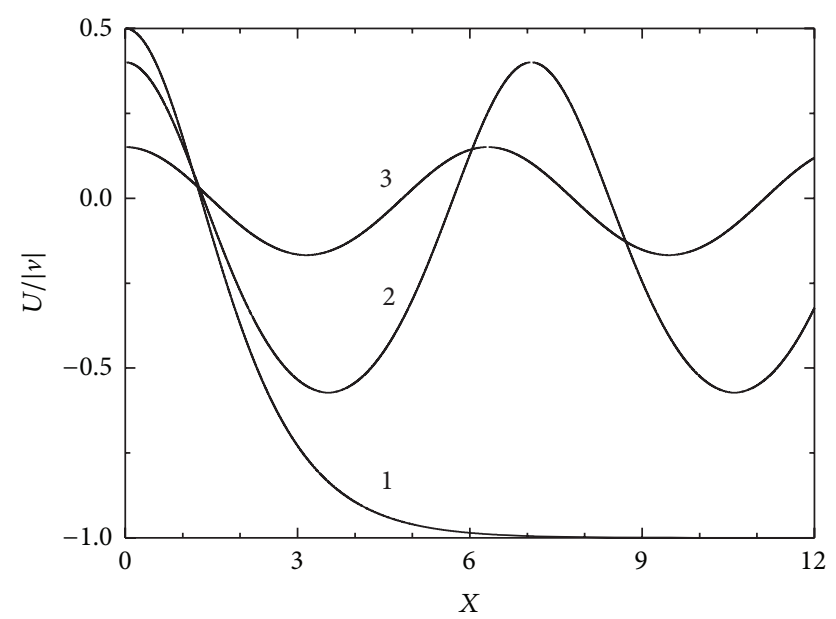

FIGURE 4: Traveling wave solutions with $v<0$ in coordinates $(X, T)$.

The solutions for $v>0$ in coordinates $(X, T)$ are illustrated in Figure 3. Curves 1 and 2 in this figure relate to curves 1 and 2 in Figure 1 . The solutions in coordinates $(X, T)$ for $v<0$ are plotted in Figure 4. Curves 1, 2, and 3 in this figure relate to curves 1, 2, and 3 in Figure 2.

On one hand, we have attained the goal; namely, we have found the solutions in new coordinates in which the solutions become one-valued functions. On the other hand, it is important that periodical solution shown by curve 1 in Figure 3, that is, the solution consisting of parabolas, becomes not periodical in new coordinates. Hence, we reveal some accordance between curve 1 in Figure 1 and curve 1 in Figure 2. This feature is important for finding the solutions by inverse scattering method [38, 43-47].

\section{Hirota Method}

Now let us define the notion "soliton" more precisely. Apart from the fact that a soliton is a stable solitary wave with particle-like properties, a soliton must possess additional properties. One property is that two such solitary waves may pass through each other without any loss of identity. Consider two solitons with different speeds, the faster one chasing the slower one. The faster soliton will eventually overtake the slower one. After the nonlinear interaction, two solitons again will emerge, with the faster one in front, and each will regain its former identity precisely. The only interaction memory will be a phase shift; each soliton will be centered at a location different from where it would have been had it traveled unimpeded. However, this property is still not sufficient in order that the solitary wave be a soliton. There are equations which possess solutions which are a nonlinear superposition of two solitary waves but which do not have all the properties enjoyed by soliton equations. A soliton equation, when it admits solitary wave solutions, must possess a solution which satisfies the "N-soliton condition" (see Section 5.3). The solitary wave with these properties defines a soliton. The term "soliton" was originally coined by Zabusky and Kruskal in 1965 [48].

One of the key properties of a soliton equation is that it has an infinite number of conservation laws. These soliton equations satisfy the Hirota condition (" $N$-soliton condition") and are exactly integrable.

The Hirota method not only gives the $N$-soliton solution, but also enables one to find a way from the Bäcklund transformation through the conservation laws and associated eigenvalue problem to the inverse scattering method. Thus the Hirota method, which can be applied only for finding solitary wave solutions or traveling wave solutions, allows us to formulate the inverse scattering method which is the most appropriate way of tackling the initial value problem (Cauchy problem). Consequently, in this case, the integrability of an equation can be regarded as proved.

5.1. The D-Operator and N-Soliton Solution. Various effective approaches have been developed to construct exact wave solutions of completely integrable equations. One of the fundamental direct methods is undoubtedly the Hirota bilinear method [14, 15, 49, 50], which possesses significant features that make it practical for the determination of multiple soliton solutions.

In the Hirota method the equation under investigation should first be transformed into the Hirota bilinear form [14]

$$
F\left(D_{X}, D_{T}\right) f \cdot f=0
$$

where $F$ is a polynomial in $D_{T}$ and $D_{X}$. Each equation has its own polynomial. The Hirota bilinear $D$-operator is defined as (see Section 5.2 in [14])

$$
\begin{gathered}
D_{T}^{n} D_{X}^{m} a \cdot b=\left(\frac{\partial}{\partial T}-\frac{\partial}{\partial T^{\prime}}\right)^{n}\left(\frac{\partial}{\partial X}-\frac{\partial}{\partial X^{\prime}}\right)^{m} a(T, X) \\
\left.\cdot b\left(T^{\prime}, X^{\prime}\right)\right|_{T=T^{\prime}, X=X^{\prime}} \cdot
\end{gathered}
$$


If the polynomial $F$ satisfies conditions (see (5.41) and (5.42) in [14])

$$
F\left(D_{X}, D_{T}\right)=F\left(-D_{X},-D_{T}\right), \quad F(0,0)=0,
$$

then the Hirota method can be applied successfully.

According to [14], the $N$-soliton solution reads as follows:

$$
f=\sum_{\mu=0,1} \exp \left[2\left(\sum_{i=1}^{N} \mu_{i} \eta_{i}+\sum_{i<j}^{(N)} \mu_{i} \mu_{j} \ln b_{i j}\right)\right],
$$

where

$$
\begin{aligned}
& b_{i j}^{2}=-\frac{F\left[2\left(k_{i}-k_{j}\right),-2\left(\omega_{i}-\omega_{j}\right)\right]}{F\left[2\left(k_{i}+k_{j}\right),-2\left(\omega_{i}+\omega_{j}\right)\right]}, \\
& \eta_{i}=k_{i} X-\omega_{i} T+\alpha_{i} .
\end{aligned}
$$

The connection between $k_{i}$ and $\omega_{i}$ is found by the dispersion relations

$$
F\left(2 k_{i},-2 \omega_{i}\right)=0, \quad i=1, \ldots, N .
$$

In (63), $\sum_{\mu=0,1}$ means the summation over all possible combinations of $\mu_{1}=0$ or $1, \mu_{2}=0$ or $1, \ldots, \mu_{N}=0$ or 1 , and $\sum_{i<j}^{(N)}$ means the summation over all possible combinations of $N$ elements under the condition $i<j$.

Moreover, for there to be an $N$-soliton solution (NSS) to (60) with $N(\geq 1)$ arbitrary, $F\left(D_{X}, D_{T}\right)$ must satisfy the " $N$ soliton condition" (NSC) [14]; namely,

$$
G^{(n)}\left(p_{1}, \ldots, p_{n}\right)=0, \quad n=1,2, \ldots, N,
$$

where

$$
G^{(1)}\left(p_{1}\right):=0
$$

and, for $n \geq 2$,

$$
\begin{gathered}
G^{(n)}\left(p_{1}, \ldots, p_{n}\right):=C \sum_{\sigma= \pm 1}\left\{F\left(\sum_{i=1}^{n} \sigma_{i} p_{i}, \sum_{i=1}^{n} \sigma_{i} \Omega_{i}\right)\right. \\
\left.\cdot \prod_{i>j}^{(n)} F\left(\sigma_{i} p_{i}-\sigma_{j} p_{j}, \sigma_{i} \Omega_{i}-\sigma_{j} \Omega_{j}\right) \sigma_{i} \sigma_{j}\right\} .
\end{gathered}
$$

In (68) $\Omega_{i}$ are given in terms of $p_{i}$ by the dispersion relations $F\left(p_{i}, \Omega_{i}\right)=0(i=1, \ldots, N), \sum_{\sigma= \pm 1}$ means the summation over all possible combinations of $\sigma_{1}= \pm 1, \sigma_{2}= \pm 1, \ldots, \sigma_{n}=$ \pm 1 , and $C$ is a function of $p_{i}$ that is independent of the summation indices $\sigma_{1}, \ldots, \sigma_{n}$.

From (67) it follows that (66) is satisfied for $n=1$. If $F(p, \Omega)=F(-p,-\Omega)$, then (66) is satisfied for $n=2$. However, whether or not (66) is satisfied for $n \geq 3$ depends on the particular form of $F(p, \Omega)$, that is, on the original equation being studied.
5.2. Bilinear Form of the Vakhnenko-Parkes Equation. In order to find soliton solutions to VPE (50)

$$
W_{X X T}+\left(1+W_{T}\right) W_{X}=0
$$

by using Hirota's method [14] we need to express (50) in Hirota form [36]. Transformation (53) of the independent variables in the original equation (11) is a key step in finding an exact explicit $N$-soliton solution to (50) by use of the Hirota method and hence an exact implicit $N$-soliton solution to (11). By taking

$$
W=6(\ln f)_{X},
$$

we find that

$$
\begin{gathered}
W_{X}=\frac{3 D_{X}^{2} f \cdot f}{f^{2}}, \\
W_{X X T}+W_{X} W_{T}=\frac{3 D_{T} D_{X}^{3} f \cdot f}{f^{2}} \\
F\left(D_{X}, D_{T}\right) f \cdot f=0, \\
F\left(D_{X}, D_{T}\right):=D_{T} D_{X}^{3}+D_{X}^{2} .
\end{gathered}
$$

and the bilinear form of the VPE is as follows:

In passing we note that the Hirota-Satsuma equation (HSE) for shallow water waves [51]

$$
-u_{t}+u_{x x t}+u u_{t}+u_{x} \int_{-\infty}^{x} u_{t} d x^{\prime}+u_{x}=0
$$

may be written as

$$
W_{x x y}+\left(1+W_{t}\right) W_{x}-W_{t}=0, \quad u=W_{x}=6(\ln f)_{x x}
$$

or in bilinear form

$$
\left(D_{t} D_{x}^{3}+D_{x}^{2}+D_{t} D_{x}\right) f \cdot f=0 .
$$

Clearly (74) and (75) are similar to, but cannot be transformed into, (50) and (72), respectively. Hence solutions to the HSE cannot be transformed into solutions of the VPE. The solution to the HSE by Hirota method is given in [51].

The Hirota method can be applied successfully if we can prove " $N$-soliton condition" (NSC) (66)-(68) for (72). Let us present this proof [37].

5.3. The "N-Soliton Condition" for the VPE. Since, for (72), we have $F(p, \Omega)=F(-p,-\Omega)$, then (66) is satisfied for $n=2$. 
With $F$ given by (72), the dispersion relations give $\Omega_{i}=$ $-1 / p_{i}$ and (66) may be written:

$$
\begin{gathered}
G^{(n)}\left(p_{1}, \ldots, p_{n}\right):=\left(\prod_{i=1}^{n} p_{i}\right) \sum_{\sigma= \pm 1}\left\{\left(\sum_{i=1}^{n} \sigma_{i} p_{i}\right)^{2}\right. \\
\cdot\left[1-\left(\sum_{i=1}^{n} \frac{\sigma_{i}}{p_{i}}\right)\left(\sum_{i=1}^{n} \sigma_{i} p_{i}\right)\right] \prod_{i>j}^{(n)}\left(\sigma_{i} p_{i}-\sigma_{j} p_{j}\right)^{2} \\
\left.\cdot\left(p_{i}^{2}+p_{j}^{2}-\sigma_{i} \sigma_{j} p_{i} p_{j}\right)\right\} .
\end{gathered}
$$

The presence of the first product term in (76) ensures that $G^{(n)}$ is a homogeneous polynomial in $p_{i}$.

In passing we remark that previous work suggests, but does not prove, that (72) does have an NSS for all $N \geq 1$. The expression for $F$ given by $(72)$ is a special case of one proposed by Ito (see Equation (B.10) in [52]). Ito claimed that this $F$ satisfies 3SC. Hietarinta [53] performed a search for bilinear equations of the form (72) that have $F$ that satisfies 3SC. One such $F$ was found to be the one given by (72). Hietarinta [54] later claimed that this $F$ also passed 4SC. The bilinear equation (72) is a special case of one given in Grammaticos et al. (see Equation (4.4) in [55]); they showed that this equation has the Painlevé property. According to Hietarinta [54] a bilinear equation that has 4SS and the Painlevé property is almost certainly integrable. All this evidence suggests that it highly likely that (72) does have an NSS for all $N \geq 1$. Here we remove any doubt by using induction to prove that condition (66) is satisfied with $G^{(n)}$ given by (76).

We need the following properties of $G^{(n)}$ (as given by (76)) for $n \geq 3$ :

(i) $\left.G^{(n)}\left(p_{1}, \ldots, p_{n}\right)\right|_{p_{1}=0} \equiv 0$.

(ii) $\left.G^{(n)}\left(p_{1}, \ldots, p_{n}\right)\right|_{p_{1}= \pm p_{2}}= \pm 24 p_{1}^{6}\left[\prod_{i=3}^{n}\left(p_{i}^{2}-p_{1}^{2}\right)^{2}\left(p_{i}^{4}+\right.\right.$ $\left.\left.p_{1}^{4}+p_{i}^{2} p_{1}^{2}\right)\right] G^{(n-2)}\left(p_{3}, \ldots, p_{n}\right)$.

(iii) $\left.G^{(n)}\left(p_{1}, \ldots, p_{n}\right)\right|_{p_{1}^{2}+p_{2}^{2} \pm p_{1} p_{2}=0}= \pm\left(p_{1} \mp p_{2}\right)\left(p_{1}^{2}-\right.$ $\left.p_{2}^{2}\right)\left(p_{1}^{2}+p_{2}^{2} \mp p_{1} p_{2}\right)\left[\prod_{i=3}^{n}\left[\left\{\left(p_{1} \pm p_{2}\right)^{2}+p_{i}^{2}\right\}^{2}-\left(p_{1} \pm\right.\right.\right.$ $\left.\left.\left.p_{2}\right)^{2} p_{i}^{2}\right]\right] G^{(n-1)}\left(p_{1} \pm p_{2}, p_{3}, \ldots, p_{n}\right)$.

(We established property (iii) by adapting the argument used to obtain equation (28) in [56] in the context of a shallow water wave equation.) Furthermore, because of $\sigma$ summation in (76), $G^{(n)}$ is an odd, symmetric function of $p_{i}$. As already noted, the condition (66) is satisfied for $n=1$ and $n=2$. We now assume that the condition is satisfied for all $n \leq m-1$, where $m \geq 3$; then the properties of $G^{(n)}$ imply that it may be factorized as follows:

$$
\begin{aligned}
& G^{(m)}\left(p_{1}, \ldots, p_{m}\right)=\left[\prod_{i=1}^{m} p_{i}\right]\left[\prod_{i>j}^{(m)}\left(p_{i}^{2}-p_{j}^{2}\right)^{2}\right. \\
& \left.\cdot\left(p_{i}^{2}+p_{j}^{2}+p_{i} p_{j}\right)\left(p_{i}^{2}+p_{j}^{2}-p_{i} p_{j}\right)\right] \\
& \cdot \widetilde{G}^{(m)}\left(p_{1}, \ldots, p_{m}\right),
\end{aligned}
$$

where $\widetilde{G}^{(m)}$ is a homogeneous polynomial. It follows that the degree of $G^{(m)}$ is at least $4 m^{2}-3 m$. On the other hand, from (76) the degree of $G^{(m)}$ is at most $2 m^{2}-m+2$. As $4 m^{2}-3 m>$ $2 m^{2}-m+2$ for $m \geq 3$, it follows that $G^{(m)} \equiv 0$. It now follows by induction that the NSC is satisfied.

5.4. The N-Soliton Solution of the VPE. With F given by (72) for the VPE

$$
W_{X X T}+\left(1+W_{T}\right) W_{X}=0
$$

the dispersion relations $(65) F\left(2 k_{i},-2 \omega_{i}\right)=0(i=1, \ldots, N)$ give $\omega_{i}=1 / 4 k_{i}$ and then

$$
\eta_{i}=k_{i}\left(X-c_{i} T\right)+\alpha_{i} \quad \text { with } c_{i}=\frac{1}{4 k_{i}^{2}} .
$$

Also, without loss of generality, we may take $k_{1}<\cdots<k_{N}$ and then

$$
b_{i j}=\frac{k_{j}-k_{i}}{k_{i}+k_{j}} \sqrt{\frac{k_{i}^{2}+k_{j}^{2}-k_{i} k_{j}}{k_{i}^{2}+k_{j}^{2}+k_{i} k_{j}}}, \quad \text { where } i<j,
$$

so that $0<b_{i j}<1$.

Consequently, the relationship (63) with (79) and (80) gives $f$ for the VPE. Finally, substitution of (63) into (70) gives the $N$-soliton solution $W(X, T)$ of the VPE [37].

However, following Moloney and Hodnett [57], it is more convenient to express $f$ in the form

$$
f=h_{i}+\widehat{h}_{i} e^{2 \eta_{i}}
$$

for a given $i$ with $1 \leq i \leq N$, where

$$
\begin{aligned}
& h_{i}=\sum_{\mu=0,1} \exp \left[2\left(\sum_{\substack{r=1 \\
(r \neq i)}}^{N} \mu_{r} \eta_{r}+\sum_{\substack{r<s \\
(r \neq i, s \neq i)}}^{(N)} \mu_{r} \mu_{s} \ln b_{r s}\right)\right], \\
& \hat{h}_{i}=\sum_{\mu=0,1} \exp \left[2\left(\sum_{\substack{r=1 \\
r \neq i}}^{N} \mu_{r} \eta_{r}+\sum_{\substack{r<s \\
r \neq i, s \neq i)}}^{(N)} \mu_{r} \mu_{s} \ln b_{r s}+\sum_{r=1}^{i-1} \mu_{r} \ln b_{r i}+\sum_{r=i+1}^{N} \mu_{r} \ln b_{i r}\right)\right] .
\end{aligned}
$$


Then we may write the $N$-soliton solution for VPE (50) in the form

$$
W=\sum_{i=1}^{N} W_{i}
$$

$$
\text { where } W_{i}=6 k_{i}\left(1+\tanh g_{i}\right), g_{i}(X, T)=\eta_{i}+\frac{1}{2} \ln \left[\frac{\widehat{h}_{i}}{h_{i}}\right] \text {. }
$$

From (83) and the relationship $U=W_{X}$, the $N$-soliton solution for VPE (51) is

$$
U=\sum_{i=1}^{N} U_{i}, \quad \text { where } U_{i}=6 k_{i} \frac{\partial g_{i}}{\partial X} \operatorname{sech}^{2} g_{i} .
$$

With $W$ and $U$ given by (83) and (84), respectively, and by using (45), (46), and (52), we may write the $N$-loop soliton solution to $\mathrm{VE}$ (11) in the parametric form:

$$
\begin{aligned}
u(x, t) & =U(t, T), \\
x & =\theta(t, T), \\
\theta(X, T) & =T+W(X, T)+x_{0} .
\end{aligned}
$$

5.5. The One-Loop Soliton Solution. The solution to (72) corresponding to one soliton is given by

$$
f=1+e^{2 \eta}, \quad \text { where } \eta=k X-\omega T+\alpha,
$$

and $k, \omega$, and $\alpha$ are constants. The dispersion relation (65) is $F(2 k,-2 \omega)=0$ from which we find that $\omega=1 / 4 k$ and then

$$
\eta=k(X-c T)+\alpha \quad \text { with } c=\frac{1}{4 k^{2}} .
$$

Substitution of (86) into (70) gives

$$
W(X, T)=6 k(1+\tanh \eta)
$$

so that

$$
U(X, T)=6 k^{2} \operatorname{sech}^{2} \eta
$$

The one-loop soliton solution to the VE is given by (85) with (88) and (89). From (85) with $v=1 / c$ we have

$$
\begin{aligned}
x-v t= & -v(X-c T) \\
& +6 k(1+\tanh [k(X-c T)+\alpha])+x_{0} .
\end{aligned}
$$

Clearly, from (89) and (90), $U(X, T)$ and $x-v t$ are related by the parameter $\chi=X-c T$ so that $u(x, t)$ is a soliton that travels with speed $v$ in the positive $x$-direction. The fact that this soliton is a loop may be shown as follows. From (54) we have $u_{x}=\phi^{-1} U_{T}$, and on using (87) and (89) we also have $\phi=1-c U$ and $U_{T}=-c U_{X}$. Hence

$$
u_{x}=-\frac{c U_{X}}{(1-c U)} \text {. }
$$

Thus, as $\chi$ goes from $\infty$ to $-\infty$ in (90), so that $x-v t$ goes from $-\infty$ to $+\infty, U_{X}$ changes sign once and remains finite whereas $u_{x}$ given by (91) changes sign three times and goes infinite twice. The one-loop soliton solution may be written in terms of the parameter $\chi$ as

$$
\begin{gathered}
u=\frac{3 v}{2} \operatorname{sech}^{2}\left(\frac{\sqrt{v} \chi}{2}\right), \\
x-v t=\tilde{x}_{0}-v \chi+3 \sqrt{v} \tanh \left(\frac{\sqrt{v} \chi}{2}\right)
\end{gathered}
$$

with $v(>0)$ and $\tilde{x}_{0}$ arbitrary. Solution (92) is essentially the one-loop soliton solution given by $(30)$ (see $[20,21]$ too).

Usually it is assumed that the value $\alpha$ is real in order that the solution $U(X, T)$ is a real function. However, the real solution is obtained also at $\alpha=-i \pi+\widetilde{\alpha}$ ( $\widetilde{\alpha}$ is real) (see Sections 9.1 and 11.2 and Appendix B). In this case the soliton solution (singular soliton solution) is discontinuous [58]

$$
U(X, T)=6 k^{2} \sinh ^{-2} \eta .
$$

5.6. The Two-Loop Soliton Solution. The solution to (72) corresponding to two solitons is given by

$$
\begin{aligned}
& f=1+e^{2 \eta_{1}}+e^{2 \eta_{2}}+b^{2} e^{2\left(\eta_{1}+\eta_{2}\right)}, \\
& \text { where } \eta_{i}=k_{i} X-\omega_{i} T+\alpha_{i}, \\
& b^{2}=-\frac{F\left[2\left(k_{1}-k_{2}\right),-2\left(\omega_{1}-\omega_{2}\right)\right]}{F\left[2\left(k_{1}+k_{2}\right),-2\left(\omega_{1}+\omega_{2}\right)\right]},
\end{aligned}
$$

and $k_{i}, \omega_{i}$, and $\alpha_{i}$ are constants. The dispersion relation is $F\left(2 k_{i},-2 \omega_{i}\right)=0$ from which we find that $\omega_{i}=1 / 4 k_{i}$ and then

$$
\eta_{i}=k_{i}\left(X-c_{i} T\right)+\alpha_{i} \quad \text { with } c_{i}=\frac{1}{4 k_{i}^{2}} .
$$

Without loss of generality we may take $k_{2}>k_{1}$ and then

$$
b=\frac{k_{2}-k_{1}}{k_{2}+k_{1}} \sqrt{\frac{k_{1}^{2}+k_{2}^{2}-k_{1} k_{2}}{k_{1}^{2}+k_{2}^{2}+k_{1} k_{2}}} .
$$

Substitution of (94) into (70) gives the two-soliton solution of the VPE. Following Hodnett and Moloney [57, 59], we may write $W(X, T)$ in the form

$$
W=W_{1}+W_{2}
$$

$$
\text { where } W_{i}=6 k_{i}\left(1+\tanh g_{i}\right) \text {, }
$$

$$
g_{2}(X, T)=\eta_{2}+\frac{1}{2} \ln \left[\frac{1+b^{2} e^{2 \eta_{1}}}{1+e^{2 \eta_{1}}}\right] .
$$

It follows that $U$ may be written:

$$
U=U_{1}+U_{2}, \quad \text { where } U_{i}=6 k_{i} \frac{\partial g_{i}}{\partial X} \operatorname{sech}^{2} g_{i} .
$$

The two-loop soliton solution to the VE is given by (85) with (98) and (100) [36]. 
5.7. Discussion of the Two-Loop Soliton Solution. We now consider the two-loop soliton solution found in Section 5.6 in more detail. First it is instructive to consider what happens in $X-T$ space.

As $c_{1}>c_{2}$, we have

$$
X-c_{2} T \longrightarrow \pm \infty
$$

as $T \longrightarrow \pm \infty$ with $X-c_{1} T$ fixed,

$$
X-c_{1} T \longrightarrow \mp \infty
$$

as $T \longrightarrow \pm \infty$ with $X-c_{2} T$ fixed.

From (99) and (100) with (101) it follows that, with $X-c_{1} T$ fixed,

$$
\begin{aligned}
& U_{1} \sim 6 k_{1}^{2} \operatorname{sech}^{2} \eta_{1} \quad \text { as } T \longrightarrow-\infty, \\
& U_{1} \sim 6 k_{1}^{2} \operatorname{sech}^{2}\left(\eta_{1}+\ln b\right) \quad \text { as } T \longrightarrow+\infty .
\end{aligned}
$$

Similarly, from (99) and (100) with (102), with $X-c_{2} T$ fixed,

$$
\begin{aligned}
& U_{2} \sim 6 k_{2}^{2} \operatorname{sech}^{2}\left(\eta_{2}+\ln b\right) \quad \text { as } T \longrightarrow-\infty, \\
& U_{2} \sim 6 k_{2}^{2} \operatorname{sech}^{2} \eta_{2} \text { as } T \longrightarrow+\infty .
\end{aligned}
$$

Hence it is apparent that, in the limits $T \rightarrow \pm \infty, U_{1}$ and $U_{2}$ may be identified as individual solitons moving with speeds $c_{1}$ and $c_{2}$, respectively, in the positive $x$-direction. In contrast to the familiar interaction of two KdV "sech squared" solitons [60], here it is the smaller soliton that overtakes the larger one.

The shifts $\Delta_{i}$ of the two solitons $U_{1}$ and $U_{2}$ in the positive $x$-direction due to the interaction are

$$
\begin{aligned}
& \Delta_{1}=-\frac{(\ln b)}{k_{1}} \\
& \Delta_{2}=\frac{(\ln b)}{k_{2}}
\end{aligned}
$$

respectively. As $\ln b<0$, the smaller soliton is shifted forwards and the larger soliton is shifted backwards. Since the "mass" of each soliton is given by $\int_{-\infty}^{\infty} U_{i} d X=12 k_{i}$, where we have used (100), and the shifts satisfy $k_{1} \Delta_{1}+k_{2} \Delta_{2}=0$, "momentum" is conserved.

Let $r:=k_{1} / k_{2}$ and recall that here we are assuming that $0<r<1$. (From (103) and (104), $r^{2}$ is the ratio of the amplitudes of the individual smaller and larger solitons.) Note that $U_{X X}\left(X_{\text {int }}, T_{\text {int }}\right)=0$ for $r=R=0.53862$, where $\left(X_{\text {int }}, T_{\text {int }}\right)$ is the center of the interaction. For $R<r<1$, we have $U_{X X}\left(X_{\text {int }}, T_{\text {int }}\right)>0$ and the two-soliton solution in $X-T$ space always has two peaks; during interaction the two humps exchange amplitudes. For $0<r<R$, we have $U_{X X}\left(X_{\text {int }}, T_{\text {int }}\right)<0$ and the two humps of the individual solitons coalesce into a single hump for part of the interaction; the smaller hump appears to pass through the larger one.

Now let us consider what happens in $x$ - $t$ space. From (85) with $v_{i}=1 / c_{i}$ we have

$$
x-v_{i} t=-v_{i}\left(X-c_{i} T\right)+W(X, T)+x_{0} .
$$

Note that in (103) taking the limits $T \rightarrow \pm \infty$ with $X-c_{1} T$ fixed is equivalent to taking the limits $X \rightarrow \pm \infty$ with $X-c_{1} T$ fixed; also note that $X=t$ from (45). Accordingly from (103) and (106) with $i=1$ we see that, in the limits $t \rightarrow \pm \infty$ with $X-c_{1} T$ fixed, $U_{1}(X, T)$ and $x-v_{1} t$ are related by the parameter $X-c_{1} T$. Similarly, from (104) and (106) with $i=2$, in the limits $t \rightarrow \pm \infty$ with $X-c_{2} T$ fixed, $U_{2}(X, T)$ and $x-v_{2} t$ are related by the parameter $X-c_{2} T$. It follows that, in the limits $t \rightarrow \pm \infty, u_{1}$ and $u_{2}$ may be identified as individual loop solitons moving with speeds $v_{1}$ and $v_{2}$, respectively, in the positive $x$-direction, where $u_{i}(x, t)=U_{i}(X, T)$. As $v_{2}>v_{1}$, the larger loop soliton overtakes the smaller loop soliton.

The shifts, $\delta_{i}$, of the two-loop solitons $u_{1}$ and $u_{2}$ in the positive $x$-direction due to the interaction may be computed from (106) as follows. From (103), as $T \rightarrow-\infty, U_{1}=U_{1 \max }=$ $6 k_{1}^{2}$, where $X-c_{1} T=-\alpha_{1} / k_{1}$; then $W_{1}=6 k_{1}$ and, by use of (101), $W_{2}=0$. Similarly, as $T \rightarrow \infty, U_{1}=U_{1 \max }=6 k_{1}^{2}$, where $X-c_{1} T=-\left(\alpha_{1}+\ln b\right) / k_{1}$; then $W_{1}=6 k_{1}$ and $W_{2}=12 k_{2}$. Use of these results in (106) with $i=1$ gives

$$
\delta_{1}=4 k_{1} \ln b+12 k_{2} .
$$

By use of (102), (104), and (106) with $i=2$, a similar calculation yields

$$
\delta_{2}=-4 k_{2} \ln b-12 k_{1}
$$

From (108) it is found that, for $0<r<1, \delta_{2}>0$ so that the larger loop soliton is always shifted forwards by the interaction. However, for $\delta_{1}$ we find that,

(a) for $r_{c}<r<1, \delta_{1}<0$ so the smaller loop soliton is shifted backwards;

(b) for $r=r_{c}$, where $r_{c}=0.88867$ is the root of $\ln b+3 / r=$ $0, \delta_{1}=0$ so the smaller loop soliton is not shifted by the interaction;

(c) for $0<r<r_{c}, \delta_{1}>0$ so the smaller loop soliton is shifted forwards.

At first sight it might seem that the behaviour in (b) and (c) contradicts conservation of "momentum." The fact that this is not so is justified as follows. By integrating (11) with respect to $x$ we find that $\int_{-\infty}^{\infty} u d x=0$; also, by multiplying (11) by $x$ and integrating with respect to $x$ we obtain $\int_{-\infty}^{\infty} x u d x=0$. Thus, in $x$ - $t$ space, the "mass" of each soliton is zero, and "momentum" is conserved whatever $\delta_{1}$ and $\delta_{2}$ may be. In particular $\delta_{1}$ and $\delta_{2}$ may have the same sign as in (c), or one of them may be zero as in (b).

Cases (a), (b), and (c) are illustrated in Figures 5, 6, and 7, respectively; in these figures $u$ is plotted against $x$ for various values of $t$. For convenience in the figures, the interactions of solitons are shown in coordinates moving with speed $\left(v_{1}+\right.$ $\left.v_{2}\right) / 2$.

5.8. Discussion of the N-Loop Soliton Solution. We now interpret the $N$-loop soliton solution found at beginning of Section 5.4 in terms of individual loop solitons [37].

First it is instructive to consider what happens in $X-T$ space. From (83) and (84) and the fact that $c_{1}>\cdots>c_{N}$ 


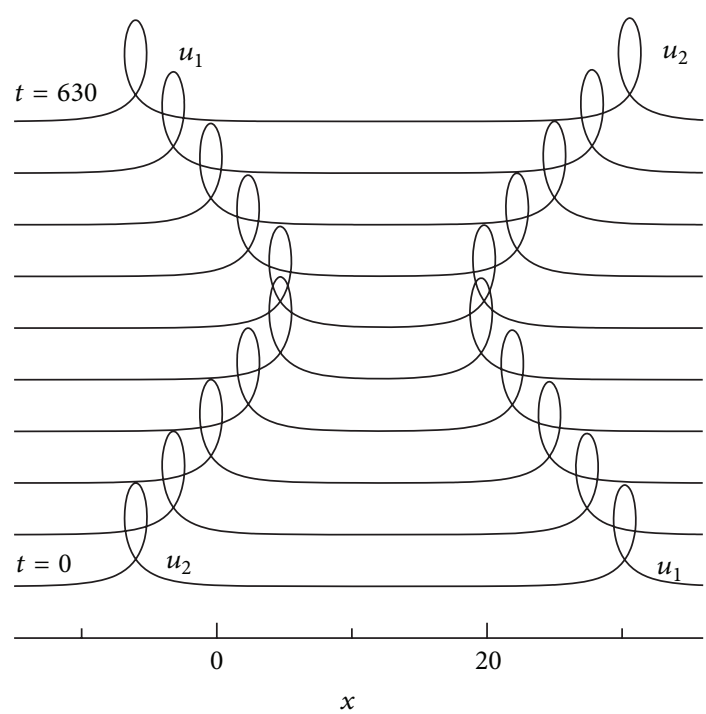

FIGURE 5: The interaction process for two-loop solitons with $k_{1}=$ 0.99 and $k_{2}=1$ so that $r=0.99$ and $\delta_{1}<0$.

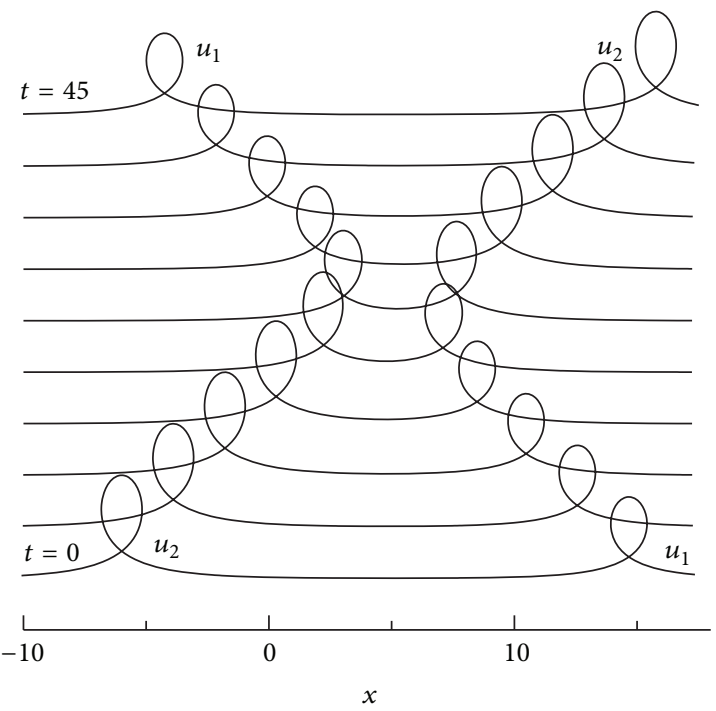

FIGURE 6: The interaction process for two-loop solitons with $k_{1}=$ 0.88867 and $k_{2}=1$ so that $r=0.88867$ and $\delta_{1}=0$.

we deduce the following behaviour: with $X-c_{i} T$ fixed and $T \rightarrow-\infty$,

$$
U_{i} \sim \begin{cases}6 k_{1}^{2} \operatorname{sech}^{2} \eta_{1}, & \text { if } i=1, \\ 6 k_{i}^{2} \operatorname{sech}^{2}\left(\eta_{i}+\sum_{r=1}^{i-1} \ln b_{r i}\right), & \text { if } 2 \leq i \leq N ;\end{cases}
$$

with $X-c_{i} T$ fixed and $T \rightarrow+\infty$,

$$
\begin{aligned}
& U_{i} \\
& \sim \begin{cases}6 k_{i}^{2} \operatorname{sech}^{2}\left(\eta_{i}+\sum_{r=i+1}^{N} \ln b_{i r}\right), & \text { if } 1 \leq i \leq N-1, \\
6 k_{N}^{2} \operatorname{sech}^{2} \eta_{N}, & \text { if } i=N .\end{cases}
\end{aligned}
$$

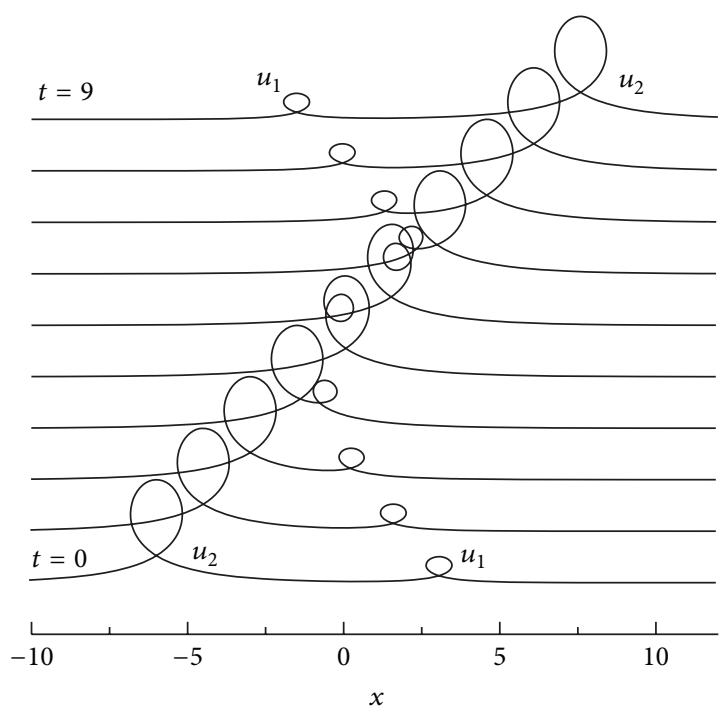

FIgURE 7: The interaction process for two-loop solitons with $k_{1}=0.5$ and $k_{2}=1$ so that $r=0.5$ and $\delta_{1}>0$.

Hence it is apparent that, in the limits $T \rightarrow \pm \infty$, each $U_{i}$ may be identified as an individual soliton moving with speed $c_{i}$ in the positive $x$-direction. Smaller solitons overtake larger ones.

The shifts, $\Delta_{i}$, of the solitons in the positive $x$-direction due to the interactions between the $N$ solitons are given by

$$
\begin{aligned}
& \Delta_{1}=-\frac{1}{k_{1}} \sum_{r=2}^{N} \ln b_{1 r}, \\
& \Delta_{i}=\frac{1}{k_{i}}\left(\sum_{r=1}^{i-1} \ln b_{r i}-\sum_{r=i+1}^{N} \ln b_{i r}\right), \quad 2 \leq i \leq N-1, \\
& \Delta_{N}=\frac{1}{k_{N}} \sum_{r=1}^{N-1} \ln b_{r N} .
\end{aligned}
$$

Since the "mass" of each soliton is given by $\int_{-\infty}^{\infty} U_{i} d X=12 k_{i}$, where we have used (84), and the shifts satisfy

$$
\sum_{i=1}^{N} k_{i} \Delta_{i}=0
$$

"momentum" is conserved.

Now let us consider what happens in $x$ - $t$ space. From (85) with $v_{i}=1 / c_{i}$ we have

$$
x-v_{i} t=-v_{i}\left(X-c_{i} T\right)+W(X, T)+x_{0} .
$$

Note that in (109) and (110) taking the limits $T \rightarrow \pm \infty$ with $X-c_{i} T$ fixed is equivalent to taking the limits $X \rightarrow \pm \infty$ with $X-c_{i} T$ fixed; also note that $X=t$ from (53). Accordingly from (109), (110), and (113), with a given $i$, we see that, in the limits $t \rightarrow \pm \infty$ with $X-c_{i}$ T fixed, $U_{i}(X, T)$ and $x-v_{i} t$ are related by 
the parameter $X-c_{i} T$. It follows that, in the limits $t \rightarrow \pm \infty$, $u_{i}$ may be identified as an individual loop soliton moving with speed $v_{i}$ in the positive $x$-direction, where $u_{i}(x, t)=U_{i}(X, T)$. As $v_{1}<\cdots<v_{N}$, larger loop solitons overtake smaller ones.
In order to calculate the shifts, $\delta_{i}$, of the loop solitons $u_{i}$ in the positive $x$-direction due to the interactions between the $N$-loop solitons, we need the following results: from (109), as $T \rightarrow-\infty, U_{i} \rightarrow U_{i \max }=6 k_{i}^{2}$, where

$$
X-c_{i} T= \begin{cases}-\frac{\alpha_{1}}{k_{1}}, & \text { for } i=1, \text { then } W \longrightarrow 6 k_{1}, \\ -\frac{\alpha_{i}}{k_{i}}-\frac{1}{k_{i}} \sum_{r=1}^{i-1} \ln b_{r i}, & \text { for } 2 \leq i \leq N, \text { then } W \longrightarrow 6 k_{i}+\sum_{r=1}^{i-1} 12 k_{r} ;\end{cases}
$$

from (110), as $T \rightarrow \infty, U_{i} \rightarrow U_{i \max }=6 k_{i}^{2}$, where

$$
X-c_{i} T= \begin{cases}-\frac{\alpha_{i}}{k_{i}}-\frac{1}{k_{i}} \sum_{r=i+1}^{N} \ln b_{i r}, & \text { for } 1 \leq i \leq N-1, \text { then } W \longrightarrow 6 k_{i}+\sum_{r=i+1}^{N} 12 k_{r} \\ -\frac{\alpha_{N}}{k_{N}}, & \text { for } i=N, \text { then } W \longrightarrow 6 k_{N} .\end{cases}
$$

Use of these results in (113) gives

$$
\begin{aligned}
& \delta_{1}=\sum_{r=2}^{N}\left(4 k_{1} \ln b_{1 r}+12 k_{r}\right), \\
& \delta_{i}=\sum_{r=i+1}^{N}\left(4 k_{i} \ln b_{i r}+12 k_{r}\right)-\sum_{r=1}^{i-1}\left(4 k_{i} \ln b_{r i}+12 k_{r}\right), \\
& \delta_{N}=-\sum_{r=1}^{N-1}\left(4 k_{N} \ln b_{r N}+12 k_{r}\right) .
\end{aligned}
$$

The three-loop soliton solution is discussed in detail in [37]. There, the interaction of three-loop solitons is illustrated in figures. The interaction process is more complicated than that for the two-loop soliton solution [36] given in Sections 5.6 and 5.7. From the three examples illustrated in [37] it is clear that several different types of interaction are possible and it is not always possible to predict what will happen on the basis of the results in [36] alone.

\section{The Vakhnenko-Parkes Equation from the Viewpoint of the Inverse Scattering Method for the KdV Equation}

Unlike the earlier Sections 5.5-5.8 where the interaction of the solitons was studied by the Hirota method $[14,15,49]$, we use now elements of the inverse scattering transform (IST) method as developed for the KdV equation [38]. The formulation of the IST method is discussed for the Vakhnenko-Parkes equation (50). It is shown that the equation system for the inverse scattering problem associated with the VPE cannot contain the isospectral Schrödinger equation. The results of this section were completed before we made appreciable progress in formulation of the IST problem for the VPE.

As we will prove later in Section 8.1, the spectral problem associated with the VPE is of third order [43, 61-63]. At first reading, the present section can be omitted. Nevertheless, methods stated here may be useful in the investigation of a new equation for which the spectral problem is unknown.

6.1. One-Soliton Solutions as Reflectionless Potentials. As was noted previously, the VE (11)

$$
\left(u_{t}+u u_{x}\right)_{x}+u=0
$$

and the KdV equation

$$
u_{t}+6 u u_{x}+u_{x x x}=0
$$

have the same hydrodynamic nonlinearity and do not contain dissipative terms; only the dispersive terms are different. The similarity between these equations indicates that, in studying the VE and the VPE (50), the application of the IST method should be possible. The IST method is the most appropriate way of tackling initial value problems. The results of applying the IST method would be useful in solving the Cauchy problem for both the VE and the VPE. The study of the VPE is of scientific interest from the viewpoint of the general problem of integrability of nonlinear equations.

The method of the IST is a powerful method as a means for solving the nonlinear differential equations. Let us recall that $\mathrm{KdV}$ equation (118) is associated with the system of the equations

$$
\begin{aligned}
\psi_{x x}+u \psi & =\lambda \psi \\
\psi_{t}+3 \lambda \psi_{x}+\psi_{x x x}+3 u \psi_{x} & =0 .
\end{aligned}
$$

Equation system (119) and (120) is a case of the IST method presented in the classic paper [1]. Since system (119) and (120) 
contains Schrödinger equation (119), we will use the elements of the IST method as applied to the KdV equation in order to analyze the VPE. The known one-soliton solution of KdV equation (118) has the form (without the time-dependence)

$$
u=2 \varrho^{2} \operatorname{sech}^{2} \varrho x .
$$

Here, as an example, we will consider the case $\varrho=1$.

The results in this paper are based on the assumption that the system of equations associated with VPE (50), which are analogous to (119) and (120), is unknown.

Now let us focus on the fact that (48) is the Schrödinger equation

$$
\frac{\partial^{2} \psi}{\partial X^{2}}-Q \psi=\lambda \psi
$$

with the eigenvalue (energy) $\lambda=0$ and potential $Q=-U$. Equation (48) determines the dependence on the coordinate $X$, and time $T$ appears here as a parameter. However, the time-dependence is determined by (47).

The known one-soliton solution of (50), which we obtained in Section 5.5, has the form

$$
U=\frac{3 v}{2} \operatorname{sech}^{2}\left(\frac{v X-T}{2 \sqrt{v}}\right)
$$

If it is not otherwise noted, for convenience here we will consider $v=4$ and $T=0$, and then (123) reduces to

$$
U=6 \operatorname{sech}^{2} X
$$

The principal fact is that both $u=2 \operatorname{sech}^{2} x$ from (121) and $U=$ $6 \operatorname{sech}^{2} X$ from (124) relate to reflectionless potentials. The general form of the reflectionless potentials is (see Section 2.4 in $[8])$

$$
u=m(m+1) \operatorname{sech}^{2} x .
$$

We have $m=1$ for the potential (121) and $m=2$ for the potential $(124)$. It is known $[8,13]$ that, for integrable nonlinear equations, reflectionless potentials generate soliton solutions (in the general case, $\mathrm{N}$-soliton solutions).

6.2. Two-Level Reflectionless Potential. Let us consider the one-soliton solution of system (119) and (120) in the framework of the IST method for the KdV equation. For this purpose let us analyze the Schrödinger equation with the potential $Q \equiv-U=-6 \operatorname{sech}^{2} X(T$ is a parameter $)$

$$
\frac{d^{2} \psi}{d X^{2}}-Q \psi=-k^{2} \psi, \quad k^{2}=-\lambda .
$$

For the scattering problem, the solution of (126) should satisfy the boundary conditions

$$
\psi(X, k)= \begin{cases}e^{-i k X}, & X \longrightarrow-\infty \\ b(k) e^{i k X}+a(k) e^{-i k X}, & X \longrightarrow+\infty,\end{cases}
$$

where $b(k)$ and $a(k)$ are the coefficients of reflection and transmission, respectively.
In Section 2.4 in [8], the original method for finding the wave-functions $\psi$ and eigenvalues for the reflectionless potential $Q_{m}=-m(m+1) \operatorname{sech}^{2} X$ was described. The general solution $y_{m}$ of (126) for the potential $Q_{m}$ connects with the general solution $Y_{0}$ for $Q_{0}=0$ by the relationship

$$
y_{m}(X, k)=\prod_{m^{\prime}=1}^{m}\left(m^{\prime} \tanh X-\frac{d}{d X}\right) Y_{0}(X, k),
$$

and then

$$
\begin{aligned}
& a(k)=\prod_{m^{\prime}=1}^{m} \frac{i k+m^{\prime}}{i k-m^{\prime}}, \\
& b(k)=0 .
\end{aligned}
$$

In our case $(m=2)(126)$ has two bound states

$$
\begin{aligned}
-i k_{1} & \equiv \kappa_{1}=1, \\
\psi_{1} & =\sqrt{\frac{3}{2}} \tanh X \operatorname{sech} X, \\
-i k_{2} & \equiv \kappa_{2}=2, \\
\psi_{2} & =\frac{\sqrt{3}}{2} \operatorname{sech}^{2} X .
\end{aligned}
$$

The wave-functions $\psi_{i}$ are normalized; that is, $\int_{-\infty}^{+\infty}\left|\psi_{i}\right|^{2} d X=$ 1 , and this conforms to the requirement used in the IST method.

Here the main difference between the VPE and the known integrable nonlinear equations appears. It is connected with the existence of only one bound state for the known equations associated with the isospectral Schrödinger equation, while for the VPE two bound states occur. Indeed, for the known integrable equations, the potential corresponding to the onesoliton solution has the following dependence on the space coordinate (see Equation (4.3.9) in [8])

$$
u(x)=2 \varrho^{2} \operatorname{sech}^{2} \varrho x .
$$

It is easy to see that this is related to the case $m=1$ in (125); that is, there is only the one bound state

$$
\begin{aligned}
& \psi=\sqrt{\frac{\varrho}{2}} \operatorname{sech} \varrho x, \\
& \psi \longrightarrow c \sqrt{\varrho} \exp (-\varrho x), \quad c=\sqrt{2}, \text { as } x \longrightarrow+\infty .
\end{aligned}
$$

6.3. Reconstruction of the One-Soliton Solution for the VPE. Keeping in mind that there is an incomplete analogy of our problem to the known integrable equations, we will try to reconstruct the potential (the solution of the VPE) from the scattering data and to find afterwards the time-dependence for the scattering data and for the one-soliton solution.

As is well known $[8,13]$, in order to reconstruct the potential for the Schrödinger equation (126), we have to know 
the scattering data. From relationships (130) we obtain, as $X \rightarrow \infty$,

$$
\begin{aligned}
& \psi_{1} \longrightarrow c_{1} e^{-\kappa_{1} X}, \quad c_{1}=\sqrt{6}, \kappa_{1}=1, \\
& \psi_{2} \longrightarrow c_{2} e^{-\kappa_{2} X}, \quad c_{2}=\sqrt{12}, \kappa_{2}=2 .
\end{aligned}
$$

Clearly, $\kappa_{1}=(1 / 2) \kappa_{2}=1$ is in agreement with (123), (124), and (121). However, we will abandon this condition, that is, $v=4$, in (131) and in the final formulas.

For convenience we reproduce the well-known procedure for the reconstruction of the potential. The function $B(X ; T)$ is constructed from the scattering data ( $T$ is the parameter)

$$
\begin{aligned}
B(X ; T)= & \sum_{m=1}^{n} c_{m}^{2}(t) e^{-\kappa_{m} X} \\
& +\frac{1}{2 \pi} \int_{-\infty}^{+\infty} b(k, T) e^{i k X} d k .
\end{aligned}
$$

In the next step the Marchenko-Gelfand-Levitan equation is to be solved [64] for the unknown $K(X, y ; T)$

$$
\begin{aligned}
& K(X, y ; T)+B(X+y ; T) \\
& \quad+\int_{X}^{+\infty} B(y+z ; T) K(X, z ; T) d z=0 .
\end{aligned}
$$

The potential is then obtained by means of the relationship

$$
-U=Q=-2 \frac{d}{d X} K(X, X ; T) .
$$

In particular, for the reflectionless potential (125), $b(k)=0$ in (127), and the solution can be found in the form

$$
K(X, y ; T)=-\sum_{m=1}^{N} c_{m}(T) \psi_{m}(X ; T) e^{-\kappa_{m} y} .
$$

This procedure, as is well known, leads to the equation system in $\psi_{m}$

$$
\mathbf{A} \Psi=\mathbf{C}
$$

where the matrix $\mathbf{A}=\left[a_{m n}\right]$ has elements

$$
a_{m n}=\delta_{m n}+c_{n}(T) c_{m}(T) \frac{e^{-X\left(\kappa_{m}+\kappa_{n}\right)}}{\kappa_{m}+\kappa_{n}},
$$

and $\boldsymbol{\Psi}=\left[\psi_{m}\right]$ and $\mathbf{C}=\left[c_{m}(T) e^{-\kappa_{m} X}\right]$ are column-vectors.

In (135)-(138), $T$ is a parameter. Although we took $T=0$ earlier, we preserve the variable $T$ in these relationships in order to use them later to find the time-dependence of the scattering data.

It is known $[8,13]$ that for a reflectionless potential the value of the determinant $\Delta=\operatorname{det}\left[a_{m n}\right]$ is sufficient for reconstructing the potential. Then (136) is reduced to

$$
\begin{aligned}
K(X, X ; T) & =\frac{d \ln |\Delta|}{d X}, \\
-U & =-2 \frac{d^{2} \ln |\Delta|}{d X^{2}} .
\end{aligned}
$$

We use (138) and (140) to obtain the one-soliton solution of the VPE. The scattering data (133) and $b(k)=0$ enable us to define the determinant

$$
\Delta=\left|\begin{array}{cc}
1+\frac{c_{1}^{2}}{2} e^{-2 X} & \frac{c_{1} c_{2}}{3} e^{-3 X} \\
\frac{c_{1} c_{2}}{3} e^{-3 X} & 1+\frac{c_{2}^{2}}{4} e^{-4 X}
\end{array}\right|=\left(1+e^{-2 X}\right)^{3}
$$

and then the potential

$$
-U=12 \frac{d}{d X}\left(\frac{e^{-2 X}}{1+e^{-2 X}}\right)=-6 \operatorname{sech}^{2} X .
$$

Thus we have repeated the standard method for reproducing the potential by means of scattering data (as yet without timedependence). It is clear from $U=W_{X}$ and (140) that

$$
W=2 K(X, X ; T) \text {. }
$$

It is noted that the determinant for the one-soliton solution of $\mathrm{KdV}$ equation (118) has the form

$$
\begin{aligned}
& \Delta=1+e^{-2 x}, \\
& u=2 \operatorname{sech}^{2} x .
\end{aligned}
$$

The interpretation of (141) is important. In the matrix, two states (133) are involved. Clearly, the time-dependence for an individual state is its own characteristic. However, since these two states relate to the common soliton, there must be a connection between them; that is, $c_{1}(T)$ and $c_{2}(T)$ must be connected. Relation (141) determines this connection.

In the first instance we considered the dependence of the potential on the space coordinate, and the time was a parameter. Let us now find the time-dependence of the scattering data $c_{1}(T)$ and $c_{2}(T)$ that enables us to find the functional dependence of the potential (124) on $T$, that is, the time-dependence of the one-soliton solution. We start from the relation (see Equation (22), Chapter 1, Section 2 in [13]):

$$
\psi(X, k ; T)=e^{-i k X}+\int_{X}^{+\infty} K(X, y ; T) e^{-i k y} d y .
$$

Hence, there is a linear operator that reduces the solution $e^{-i k X}$ of the Schrödinger equation with null potential $Q=$ 0 to the solution of this equation with the potential $U(X)$. The function $K(X, y ; T)$ is the kernel of the transformation operator.

We write (145) for $k=0$; this procedure is correct and an appropriate theorem has been proved (see Section 3.3 in [8]):

$$
\psi(X, k=0 ; T)=1+\int_{X}^{+\infty} K(X, y ; T) d y .
$$

Clearly, $\psi(X, k=0 ; T)=\varphi(X, T)$, where $\varphi(X, T)$ satisfies the equation system (47) and (48). Taking into account (136) and (146), we obtain from the relationship (47):

$$
1+\int_{X}^{+\infty} K(X, y ; T) d y=2 \frac{\partial K(X, X, T)}{\partial T}+C
$$


Since this equation must be valid at arbitrary $X$, and taking into account that the function $K(X, y ; T) \rightarrow 0$ at $|X| \rightarrow$ $\infty$, we define the constant of integration $C=1$. We write, once again, $K(X, y ; T)$ as (137), because the potential is reflectionless, and we obtain from (147)

$$
\begin{aligned}
& \sum_{m=1}^{2} \frac{c_{m}(T)}{\kappa_{m}} \psi_{m}(X ; T) e^{-\kappa_{m} X} \\
& \quad=2 \sum_{m=1}^{2} \frac{\partial c_{m}(T) \psi_{m}(X ; T)}{\partial T} e^{-\kappa_{m} X}
\end{aligned}
$$

In this equation we must substitute the values $\psi_{m}$ that are the solution of system (138). Here we consider the values $c_{m}$ already as functions of $T$; that is, $c_{m}=c_{m}(T)$. For example $\psi_{1}$ is given by

$$
\begin{aligned}
\psi_{1} & =\Delta^{-1}\left(c_{1} e^{-\kappa_{1} X}+\frac{c_{1} c_{2}^{2}}{2 \kappa_{2}} e^{-\left(\kappa_{1}+2 \kappa_{2}\right) X}\right. \\
& \left.-\frac{c_{1} c_{2}^{2}}{\kappa_{1}+\kappa_{2}} e^{-\left(\kappa_{1}+2 \kappa_{2}\right) X}\right) .
\end{aligned}
$$

Here $\Delta$ is the determinant (141) with time-dependence of $c_{m}=c_{m}(T)$. We can calculate the following terms which are required for $(148)\left(\kappa_{1}=1, \kappa_{2}=2\right)$ :

$$
\begin{aligned}
& \sum_{m=1}^{2} \frac{c_{m}(T)}{\kappa_{m}} \psi_{m}(X ; T) e^{-\kappa_{m} X} \\
& \quad=\Delta^{-1}\left(c_{1}^{2} e^{-2 X}+\frac{1}{2} c_{2}^{2} e^{-4 X}\right), \\
& \sum_{m=1}^{2} c_{m}(T) \psi_{m}(X ; T) e^{-\kappa_{m} X} \\
& \quad=\Delta^{-1}\left(c_{1}^{2} e^{-2 X}+c_{2}^{2} e^{-4 X}+\frac{1}{12} c_{1}^{2} c_{2}^{2} e^{-6 X}\right) .
\end{aligned}
$$

Then, substituting (150) into (148) and equating to zero the coefficients of $e^{-2 j X},(j=1, \ldots, 6)$, we obtain the system of differential equations for $c_{m}(T)(m=1,2)$

$$
\begin{aligned}
& e^{-2 X}:\left(c_{1}^{2}\right)^{\prime}=\frac{1}{2} c_{1}^{2}, \\
& e^{-4 X}:\left(c_{2}^{2}\right)^{\prime}=\frac{1}{4}\left(c_{2}^{2}+c_{1}^{4}\right), \\
& e^{-6 X}: \frac{1}{3}\left(c_{1}^{2} c_{2}^{2}\right)^{\prime}+c_{1}^{2}\left(c_{2}^{2}\right)^{\prime}-c_{2}^{2}\left(c_{1}^{2}\right)^{\prime}=c_{1}^{2} c_{2}^{2}, \\
& e^{-8 X}: c_{1}^{2}\left(c_{1}^{2} c_{2}^{2}\right)^{\prime}-c_{1}^{2} c_{2}^{2}\left(c_{1}^{2}\right)^{\prime}=\frac{1}{4}\left(c_{1}^{4} c_{2}^{2}+9 c_{2}^{4}\right),
\end{aligned}
$$

$$
\begin{aligned}
& e^{-10 X}: c_{2}^{2}\left(c_{1}^{2} c_{2}^{2}\right)^{\prime}-c_{1}^{2} c_{2}^{2}\left(c_{2}^{2}\right)^{\prime}=\frac{1}{2} c_{1}^{2} c_{2}^{4}, \\
& e^{-12 X}: c_{1}^{2} c_{2}^{2}\left(c_{1}^{2} c_{2}^{2}\right)^{\prime}=c_{1}^{2} c_{2}^{2}\left(c_{1}^{2} c_{2}^{2}\right)^{\prime},
\end{aligned}
$$

where the prime denotes the derivative with respect to time $T$.

Equation system (151) is an overdetermined one; only first two equations are independent. Consequently, we solve them with initial conditions $c_{1}^{2}(0)=6, c_{2}^{2}(0)=12$. At first, we write the general solution of system (151):

$$
\begin{aligned}
& c_{1}^{2}(T)=r_{1} e^{T / 2}, \\
& c_{2}^{2}(T)=r_{2} e^{T / 4}+\frac{1}{3} r_{1}^{2} e^{T},
\end{aligned}
$$

where $r_{1}, r_{2}$ are arbitrary constants. Hence, in the general case, the time-dependence of the first and second states is different. Nevertheless, we have $r_{2} \equiv 0$ due to the relationship between $c_{1}(0)$ and $c_{2}(0)$ and then

$$
\begin{gathered}
c_{1}^{2}(T)=c_{1}^{2}(0) e^{T / 2}=6 e^{T / 2}, \\
c_{2}^{2}(T)=\frac{1}{3} c_{1}^{4}(0) e^{T}=12 e^{T} .
\end{gathered}
$$

Thus, the time-dependences satisfy the condition $c_{1}^{2}(T) /$ $c_{2}(T)=$ const. Indeed, if the time-dependence is as in (153), the determinant (141) can be rewritten as a perfect cube; namely,

$$
\Delta=\left(1+e^{-2(X-T / 4)}\right)^{3}
$$

For convenience, up to this point we have used $\kappa_{1}=1, \kappa_{2}=2$. Now we return one arbitrary parameter $\kappa_{1}$ (with $\kappa_{2}=2 \kappa_{1}$ ) and rename it as $\alpha \equiv \kappa_{1}$, and then we obtain

$$
\Delta=\left\{1+\exp \left[-2 \alpha\left(X-\frac{T}{4 \alpha^{2}}\right)\right]\right\}^{3} .
$$

The potential for the one-soliton solution can easily be found by (140):

$$
\begin{aligned}
& U=2 \frac{d^{2} \ln |\Delta|}{d X^{2}}=6 \alpha^{2} \operatorname{sech}^{2} \Theta \\
& \Theta=\alpha\left(X-X_{0}-\frac{T}{4 \alpha^{2}}\right) .
\end{aligned}
$$

This is one-soliton solution for the VPE.

For reference we give the complete equations for finding the solution of VE (11) in terms of the initial variables $x, t$ 
(for convenience $T$ is renamed as $\mu \equiv T$ because here $\mu$ is a parameter):

$$
\begin{aligned}
\frac{\partial}{\partial x}\left(\frac{\partial}{\partial t}+u \frac{\partial}{\partial x}\right) u+u & =0 \\
u & =\left(\frac{\partial W}{\partial t}\right)_{\mu}, \\
x & =x_{0}+\mu+W, \\
W & =2\left(\frac{\partial \ln |\Delta|}{\partial t}\right)_{\mu}, \\
\Delta & =\left(1+q^{2}\right)^{3}, \\
q & =\exp (-\Theta), \\
\Theta & =\alpha\left(t-\frac{\mu-\mu_{0}}{4 \alpha^{2}}\right), \\
\alpha & =\text { const. } \\
\mu_{0} & =\text { const. }
\end{aligned}
$$

Thus we have obtained the one-soliton solution of the VE as well as the VPE using elements of the IST method for the $\mathrm{KdV}$ equation. The proposed method is also applicable for finding the two-soliton solution. It is likely that this procedure will shed light upon the formulation of the IST problem that enables one to make progress in the study of the Cauchy problem for the VE (11).

6.4. Two-Soliton Solution. Let us consider the two-soliton solution for VE (11). The key for constructing this solution is the value which is assigned to determinant (158) in the onesoliton solution. For information we rewrite the values (159) once again

$$
\begin{aligned}
& \Delta=\left(1+q^{2}\right)^{3} \\
& q=\exp \left[-\alpha\left(t-\frac{\mu-\mu_{0}}{4 \alpha^{2}}\right)\right] .
\end{aligned}
$$

It can be seen that there is some analogy to the one-soliton solution of KdV equation (144); namely,

$$
\begin{aligned}
& \Delta=1+q^{2} \\
& q=\exp \left(\alpha x-4 \alpha^{3} t\right) .
\end{aligned}
$$

Moreover, as we noted, the potentials corresponding to the one-soliton solution,

(a) for the $\operatorname{VPE}(T=0, \alpha=1)$

$$
U=6 \operatorname{sech}^{2} X
$$

(b) for the $\mathrm{KdV}$ equation $(t=0, \varrho=1)$

$$
u=2 \operatorname{sech}^{2} x,
$$

differ from each other by their coefficients. Bearing in mind (136) and that $K=\partial \ln |\Delta| / \partial X$ (see (140)), one can see that coefficient 6 in (163), in contrast to coefficient 2 in (164), is generated by exponent 3 in relationship (161).

Now, if it is recalled that the two-soliton solution for the $\mathrm{KdV}$ has the form [15]

$$
\begin{aligned}
\widetilde{F} & =\Delta=1+q_{1}^{2}+q_{2}^{2}+\widetilde{A}_{12} q_{1}^{2} q_{2}^{2}, \\
\widetilde{A}_{12} & =\frac{\left(\alpha_{1}-\alpha_{2}\right)^{2}}{\left(\alpha_{1}+\alpha_{2}\right)^{2}}, \\
q_{i} & =\exp \left[\alpha_{i}\left(x-x_{0 i}\right)-4 \alpha_{i}^{3} t\right],
\end{aligned}
$$

we can expect that the two-soliton solution for VE can be found in form (158) with the following value of $F$ instead of $\Delta$ in relation (140):

$$
\begin{aligned}
& F=\left(1+q_{1}^{2}+q_{2}^{2}+A_{12} q_{1}^{2} q_{2}^{2}\right)^{3}, \\
& q_{i}=\exp \left[-\alpha_{i}\left(t-\frac{\mu-\mu_{i}}{4 \alpha_{i}^{2}}\right)\right] .
\end{aligned}
$$

The value $A_{12}$ is to be determined. It should be noted that $F$ is not equal to the determinant $\Delta$ of the matrix in (138) which is constructed from four states with $q_{1}, q_{1}^{2}, q_{2}, q_{2}^{2}$, (each soliton has two bound states (130))

$$
\Delta=\left|\begin{array}{cccc}
1+3 q_{1}^{2} & 2 \sqrt{2} q_{1}^{3} & \frac{6 \sqrt{\alpha_{1} \alpha_{2}}}{\alpha_{1}+\alpha_{2}} q_{1} q_{2} & \frac{6 \sqrt{2 \alpha_{1} \alpha_{2}}}{\alpha_{1}+2 \alpha_{2}} q_{1} q_{2}^{2} \\
2 \sqrt{2} q_{1}^{3} & 1+3 q_{1}^{4} & \frac{6 \sqrt{2 \alpha_{1} \alpha_{2}}}{2 \alpha_{1}+\alpha_{2}} q_{1}^{2} q_{2} & \frac{6 \sqrt{\alpha_{1} \alpha_{2}}}{\alpha_{1}+\alpha_{2}} q_{1}^{2} q_{2}^{2} \\
\frac{6 \sqrt{\alpha_{1} \alpha_{2}}}{\alpha_{1}+\alpha_{2}} q_{1} q_{2} & \frac{6 \sqrt{2 \alpha_{1} \alpha_{2}}}{2 \alpha_{1}+\alpha_{2}} q_{1}^{2} q_{2} & 1+3 q_{2}^{2} & 2 \sqrt{2} q_{2}^{3} \\
\frac{6 \sqrt{2 \alpha_{1} \alpha_{2}}}{\alpha_{1}+2 \alpha_{2}} q_{1} q_{2}^{2} & \frac{6 \sqrt{\alpha_{1} \alpha_{2}}}{\alpha_{1}+\alpha_{2}} q_{1}^{2} q_{2}^{2} & 2 \sqrt{2} q_{2}^{3} & 1+3 q_{2}^{4}
\end{array}\right| .
$$


If the relation $F=\Delta$ were true, we would have $A_{12}=\widetilde{A}_{12}$. Moreover, these conditions would lead us to the statement that the problem for scattering data for VE (11) should connect with the isospectral Schrödinger equation. This statement was made in the paper by Hirota and Satsuma [49] as well as in the monograph by Newell (see Chapters 3 and 4 in [16]). However, because $F \neq \Delta$ and $A_{12} \neq \widetilde{A}_{12}$, we can state that the equation system for the IST problem associated with the VPE (50) does not contain the isospectral Schrödinger equation.

The value $A_{12}$ for (166) can be determined in the following way. The functional relation (166), with $A_{12}$ regarded as unknown, is substituted into (158) and then into (50). Equating to zero the coefficients of $\exp \left[-2\left(i \alpha_{1}+j \alpha_{2}\right) X\right],(i, j=$ $0, \ldots, 4, i+j \neq 0)$, we obtain a system of equations in one unknown $A_{12}$. It turns out that the equations are dependent. As a result we obtain

$$
A_{12}=\frac{\left(\alpha_{1}-\alpha_{2}\right)^{2}}{\left(\alpha_{1}+\alpha_{2}\right)^{2}} \cdot \frac{\alpha_{1}^{2}+\alpha_{2}^{2}-\alpha_{1} \alpha_{2}}{\alpha_{1}^{2}+\alpha_{2}^{2}+\alpha_{1} \alpha_{2}} .
$$

Thus the relationships (158), (166), and (168) are the exact two-soliton solution of the VE (11). In terms of $x$ and $t$, we have

$$
\begin{aligned}
& \frac{\partial}{\partial x}\left(\frac{\partial}{\partial t}+u \frac{\partial}{\partial x}\right) u+u=0, \\
& u=\left(\frac{\partial W}{\partial t}\right)_{\mu} \\
& x=x_{0}+\mu+W \\
& W=2\left(\frac{\partial \ln |F|}{\partial t}\right)_{\mu} \\
& F=\left(1+q_{1}^{2}+q_{2}^{2}+A_{12} q_{1}^{2} q_{2}^{2}\right)^{3}, \\
& q_{i}=\exp \left(-\Theta_{i}\right) \\
& A_{12}=\frac{\left(\alpha_{1}-\alpha_{2}\right)^{2}}{\left(\alpha_{1}+\alpha_{2}\right)^{2}} \cdot \frac{\alpha_{1}^{2}+\alpha_{2}^{2}-\alpha_{1} \alpha_{2}}{\alpha_{1}^{2}+\alpha_{2}^{2}+\alpha_{1} \alpha_{2}} \\
& \Theta_{i}=\alpha_{i} t-\frac{\mu-\mu_{i}}{4 \alpha_{i}}, \\
& \alpha_{i}=\text { const., } \\
& \mu_{i}=\text { const. }
\end{aligned}
$$

Function $W(X, T)$ in space $(X, T)$ is two-soliton solution for the VPE.

An equivalent result has been obtained, independently of the method presented here, in Section 5.6 by the means of the Hirota method $[14,15,49]$ in terms of other variables.

6.5. Remarks about This Section. The main result of Section 6 is that we have obtained a way of applying the IST method to the VPE. Keeping in mind that the IST is the most appropriate way of tackling the initial value problem, one has to formulate the associated eigenvalue problem. We have proved that the equation system for the IST problem associated with the VPE does not contain the isospectral Schrödinger equation. Nevertheless, the analysis of the VPE in the context of the isospectral Schrödinger equation allowed us to obtain the two-soliton solution. Thus the results stated here may be useful in the investigation of a new equation for which the spectral problem is unknown.

Historically, once this investigation was completed, we were able to make some progress in the formulation of the IST for the VPE. In Section 8.1 we will prove that the spectral problem associated with the VPE is of third order.

\section{Bäcklund Transformation and Conservation Laws for the VPE}

In Section 5.1 we wrote the VPE (50)

$$
W_{X X T}+\left(1+W_{T}\right) W_{X}=0
$$

in Hirota bilinear form (72)

$$
\left(D_{T} D_{X}^{3}+D_{X}^{2}\right) f \cdot f=0 .
$$

This enabled us to obtain the $N$-soliton solution of the VPE. Moreover, it turns out that a Bäcklund transformation follows from the bilinear form of the nonlinear evolution equation [43].

The definition of a Bäcklund transformation which was given by Rund in [65] is now the generally accepted one. Let $u(x, t)$ and $\widetilde{u}(x, t)$ satisfy the partial differential equations $E(u)=0$ and $D(\widetilde{u})=0$, respectively. Then the set of relations $R_{i}((u),(\widetilde{u}),(\zeta))=0(j=1, \ldots, n)$, where $(u)$ and $(\widetilde{u})$ denote strings, not necessarily of equal length, consisting of $u, \widetilde{u}$ and their various partial derivatives, is called a Bäcklund transformation if these relations ensure that $\widetilde{u}$ satisfies $D(\widetilde{u})=$ 0 whenever $u$ satisfies $E(u)=0$ and vice versa. If $u$ and $\tilde{u}$ satisfy the same equation, the adjective "auto" is inserted in front of Bäcklund transformation.

The main significance of Bäcklund transformations is that they have typically associated nonlinear superposition principles whereby infinite sequences of solutions to nonlinear equations may be generated by purely algebraic procedures. A Bäcklund transformation achieves the passage between different solution types, whether it is a one-soliton, twosoliton, bound state, and so forth. Multisoliton solutions of many important nonlinear evolution equations can thereby be constructed. We will show that a special form of the Bäcklund transformation suggested by Hirota [66] is a key for finding an infinite number of conservation laws as well as allowing one to formulate the inverse scattering problem.

Thus, the next step in the investigation of nonlinear evolution equations should be directed to obtaining the bilinear form of the Bäcklund transformation from the bilinear form of the nonlinear equation.

7.1. Bäcklund Transformation in Bilinear Form. Now we present a Bäcklund transformation for VPE (50) written in the bilinear form (72). This type of Bäcklund transformation 
was first introduced by Hirota [66] and has the advantage that the transformation equations are linear with respect to each dependent variable. This Bäcklund transformation is easily transformed to the ordinary one.

We follow the method developed in [66]. First we define $P$ as follows:

$$
\begin{aligned}
P & :=2\left\{\left[\left(D_{T} D_{X}^{3}+D_{X}^{2}\right) f^{\prime} \cdot f^{\prime}\right] f f\right. \\
& \left.-f^{\prime} f^{\prime}\left[\left(D_{T} D_{X}^{3}+D_{X}^{2}\right) f \cdot f\right]\right\},
\end{aligned}
$$

where $f \neq f^{\prime}$. We aim to find a pair of equations such that each equation is linear in each of the dependent variables $f$ and $f^{\prime}$ and such that together $f$ and $f^{\prime}$ satisfy $P=0$. (It then follows that if $f$ is a solution of (72) then so is $f^{\prime}$ and vice versa.) The pair of equations is the required Bäcklund transformation.

We show that the Bäcklund transformation is given by pair of the equations

$$
\begin{array}{r}
\left(D_{X}^{3}-\lambda\right) f^{\prime} \cdot f=0, \\
\left(3 D_{X} D_{T}+1+\mu D_{X}\right) f^{\prime} \cdot f=0,
\end{array}
$$

where $\lambda=\lambda(X)$ is an arbitrary function of $X$ and $\mu=\mu(T)$ is an arbitrary function of $T$.

We prove that together $f$ and $f^{\prime}$, as determined by (173) and (174), satisfy $P=0$ as follows. By using the identities (VII.3) and (VII.4) and Equation (5.86) from [14] we may express $P$ in the following form:

$$
\begin{aligned}
P= & D_{T}\left[\left(D_{X}^{3} f^{\prime} \cdot f\right) \cdot\left(f^{\prime} f\right)-3\left(D_{X}^{2} f^{\prime} \cdot f\right)\right. \\
& \left.\cdot\left(D_{X} f^{\prime} \cdot f\right)\right]+D_{X}\left[3\left(D_{T} D_{X}^{2} f^{\prime} \cdot f\right) \cdot\left(f^{\prime} f\right)\right. \\
& -6\left(D_{X} D_{T} f^{\prime} \cdot f\right) \cdot\left(D_{X} f^{\prime} \cdot f\right)-3\left(D_{X}^{2} f^{\prime} \cdot f\right) \\
& \left.\cdot\left(D_{T} f^{\prime} \cdot f\right)+4\left(D_{X} f^{\prime} \cdot f\right) \cdot\left(f^{\prime} f\right)\right] .
\end{aligned}
$$

We can rewrite $P$ in the following form

$$
\begin{aligned}
P= & 4 D_{T}\left(\left\{D_{X}^{3}-\lambda(X)\right\} f^{\prime} \cdot f\right) \cdot\left(f^{\prime} f\right) \\
& -4 D_{X}\left(\left\{3 D_{T} D_{X}+1+\mu(T) D_{X}\right\} f^{\prime} \cdot f\right) \\
& \cdot\left(D_{X} f^{\prime} \cdot f\right),
\end{aligned}
$$

if we use the following identities

$$
\begin{aligned}
& D_{X}^{3}\left[\left(D_{T} f^{\prime} \cdot f\right) \cdot\left(f f^{\prime}\right)\right]=D_{T}\left[\left(D_{X}^{3} f^{\prime} \cdot f\right) \cdot\left(f f^{\prime}\right)\right. \\
& \left.\quad-3\left(D_{X}^{2} f^{\prime} \cdot f\right) \cdot\left(D_{X} f^{\prime} \cdot f\right)\right], \\
& 4 D_{T}\left(D_{X}^{2} f^{\prime} \cdot f\right) \cdot\left(D_{X} f^{\prime} \cdot f\right)=D_{X}\left[\left(D_{T} D_{X}^{2} f^{\prime} \cdot f\right)\right. \\
& \quad \cdot\left(f^{\prime} f\right)+2\left(D_{T} D_{X} f^{\prime} \cdot f\right) \cdot\left(D_{X} f^{\prime} \cdot f\right) \\
& \left.-\left(D_{X}^{2} f^{\prime} \cdot f\right) \cdot\left(D_{T} f^{\prime} \cdot f\right)\right]-D_{X}^{3}\left(D_{T} f^{\prime} \cdot f\right) \\
& \quad \cdot\left(f^{\prime} f\right) .
\end{aligned}
$$

Identities (177) and (178) come from

$$
\begin{aligned}
\exp \left(D_{1}\right)\left[\exp \left(D_{2}\right) f^{\prime} \cdot f\right] \cdot\left[\exp \left(D_{3}\right) f^{\prime} \cdot f\right] \\
=\exp \left(\frac{1}{2}\left\{D_{2}-D_{3}\right\}\right) \\
\cdot\left[\exp \left\{\frac{1}{2}\left(D_{2}+D_{3}\right)+D_{1}\right\} f^{\prime} \cdot f\right] \\
\cdot\left[\exp \left\{\frac{1}{2}\left(D_{2}+D_{3}\right)-D_{1}\right\} f^{\prime} \cdot f\right]
\end{aligned}
$$

which is Equation (5.83) in [14], where $D_{i}:=\varepsilon_{i} D_{X}+\delta_{i} D_{T}$. In the order $\varepsilon_{1}^{3} \delta_{3}$, (179) yields (177), and in the order $\delta_{1} \varepsilon_{2}^{2} \varepsilon_{3}$, (179) yields (178). From (176) it follows that if (173) and (174) hold then $P=0$ as required.

Thus we have proved that the pair of (173) and (174) constitute a Bäcklund transformation in bilinear form for (72). Separately these equations appear as part of the Bäcklund transformation for other nonlinear evolution equations. For example, (173) is the same as one of the equations that is part of the Bäcklund transformation for a higher order $\mathrm{KdV}$ equation (see Equation (5.139) in [14]), and (175) is similar to Equation (5.132) in [14] that is part of the Bäcklund transformation for a model equation for shallow water waves.

The inclusion of $\mu$ in the operator $3 D_{T}+\mu$ which appears in (177) corresponds to a multiplication of $f$ and $f^{\prime}$ by terms of the form $e^{g(T)}$ and $e^{g^{\prime}(T)}$, respectively; however, this has no effect on $W$ or $W^{\prime}$ because, from $(70), W=6(\ln f)_{X}$. Hence, without loss of generality, we may take $\mu=0$ in (174) if we wish.

7.2. Bäcklund Transformation in Ordinary Form. Following the procedure given in $[14,67]$, we can rewrite the Bäcklund transformation in ordinary form in terms of the potential $W=\int_{-\infty}^{X} U d X^{\prime}$ given by (49). In new variables defined by

$$
\phi=\ln \frac{f^{\prime}}{f}, \quad \rho=\ln f^{\prime} f
$$

(173) and (174) have the form

$$
\begin{aligned}
& \phi_{X X X}+3 \phi_{X} \rho_{X X}+\phi_{X}^{3}-\lambda=0, \\
& 3\left(\rho_{X T}+\phi_{X} \phi_{T}\right)+1+\mu \phi_{X}=0,
\end{aligned}
$$

respectively, where we have used results similar to (XI.1)(XI.3) in [14]. From the definitions (70) and (180), different solutions $W, W^{\prime}$ of VPE (50) are related to $\phi$ and $\rho$ by

$$
\begin{aligned}
& W^{\prime}-W=6 \phi_{X}, \\
& W^{\prime}+W=6 \rho_{X} .
\end{aligned}
$$


Substitution of (183) into (181) and (182) with $\mu=0$ leads to

$$
\begin{aligned}
& \left(W^{\prime}-W\right)_{X X}+\frac{1}{2}\left(W^{\prime}-W\right)\left(W^{\prime}+W\right)_{X}+\frac{1}{36}\left(W^{\prime}\right. \\
& -W)^{3}-6 \lambda=0, \\
& 3 \lambda\left(W^{\prime}-W\right)_{T} \\
& +\left[\left(1-W_{T}\right)\left(\left(W^{\prime}-W\right)_{X}+\frac{1}{6}\left(W^{\prime}-W\right)^{2}\right)\right. \\
& \left.\quad-W_{X T}\left(W^{\prime}-W\right)\right]_{X}=0,
\end{aligned}
$$

respectively. The required Bäcklund transformation in ordinary form is given by (184) and (185).

Thus, by using VPE as an example, we have traced how the bilinear and ordinary forms of the Bäcklund transformation can be found from the bilinear form of an evolution equation.

7.3. The Infinite Sequence of Conservation Laws. An important property of a soliton equation is that it has conservation laws. The existence of an infinite number of conserved quantities is associated with the integrability of an equation [16].

A systematic way to derive higher conservation laws via the Bäcklund transformation has been developed by Satsuma; he applied it to the KdV equation [68]. Later Satsuma and Kaup [67] applied the method to a higher order KdV equation. Following [68], from the Bäcklund transformation we now construct the recurrence formula which gives the infinite sequence of conserved quantities for the VPE. An infinite sequence of conservation laws having the form

$$
\frac{\partial I_{n}}{\partial T}+\frac{\partial F_{n}}{\partial X}=0
$$

provides, in most cases, a corresponding sequence of integrals of motion given by the functionals $\int I_{n} d X$. Let us rewrite (184) (one of the Bäcklund transformation equations) in the form

$$
\begin{array}{r}
W^{\prime}-W \\
=6 \zeta \sqrt[3]{1-\frac{1}{6 \zeta^{3}}\left(\left(W^{\prime}-W\right)_{X X}+\frac{1}{2}\left(W^{\prime}-W\right)\left(W^{\prime}+W\right)_{X}\right)}, \\
\zeta^{3}=\lambda .
\end{array}
$$

Assuming $1 /|\zeta|$ is small, we may consider (187) to be an infinitesimal transformation from $W$ to $W^{\prime}$. Indeed, in the first approximation $W^{\prime} \simeq W+6 \zeta$ and the next approximation with respect to $|\zeta|^{-1}$

$$
W^{\prime}=W+6 \zeta+\frac{1}{6 \zeta} I_{1}
$$

Thus, we put $W$ in the form

$$
W^{\prime}=W+6 \zeta+\sum_{n=1}^{\infty} \frac{1}{6^{n} \zeta^{n}} I_{n}\left(W, W_{X}, W_{X X}, \ldots\right)
$$

Substituting (189) into (184), and equating the coefficients for the higher powers of $1 /|\zeta|$, we have

$$
\begin{aligned}
O\left(\zeta^{1}\right): I_{1}= & -2 W_{X}, \\
O\left(\zeta^{0}\right): I_{2}= & 2 W_{X X} \\
O\left(\zeta^{-1}\right): I_{3}= & -\frac{4}{3} W_{X X X}, \\
O\left(\zeta^{-2}\right): I_{4}= & \frac{2}{3} W_{X X X X}, \\
O\left(\zeta^{-3}\right): I_{5}= & -\frac{2}{9} W_{X X X X X}+\frac{1}{9}\left(W_{X}^{2}\right)_{X X}-\frac{2}{9} W_{X X}^{2} \\
& +\frac{2}{27} W_{X}^{3} .
\end{aligned}
$$

The general recursion relations for $n \geq 5$ are as follows:

$$
\begin{aligned}
I_{n}= & -\frac{1}{3} I_{n-2, X X}-I_{n-1, X}-\frac{1}{6} \sum_{i=1}^{n-3} I_{i} I_{n-i-2, X}-\frac{1}{3} W_{X} I_{n-2} \\
& -\frac{1}{6} \sum_{i=1}^{n-2} I_{i} I_{n-i-1}-\frac{1}{108} \sum_{i+j+l=n-2} I_{i} I_{j} I_{l} .
\end{aligned}
$$

The fact that these quantities are the conserved densities can be shown as follows. Let us calculate the integral $\int\left(W^{\prime}-\right.$ $W-6 \zeta)_{T} d X$ with suitable boundary conditions. Taking into account (189), we have

$$
\begin{aligned}
& \left(\int\left(W^{\prime}-W-6 \zeta\right) d X\right)_{T}=\left[\frac{1}{6^{n} \zeta^{n}} \sum_{i=1}^{\infty}\left(\int I_{n} d X\right)\right]_{T} \\
& \quad=0 .
\end{aligned}
$$

Thus we deduce that the VPE has an infinite sequence of conservation laws.

\section{The Inverse Scattering Method for the VPE}

The inverse scattering transform (IST) method is arguably the most important discovery in the theory of solitons. The method enables one to solve the initial value problem for a nonlinear evolution equation. Moreover, it provides a proof of the complete integrability of the equation.

The idea of the inverse scattering method was first introduced for the KdV equation [1] and subsequently developed for the nonlinear Schrödinger equation [13], the $\mathrm{mKdV}$ equation $[69,70]$, the sine-Gordon equation $[12,71]$, and the equation of motion for a one-dimensional exponential lattice (Toda lattice) [72]. It is to be remarked that the inverse method is a unique theory whereby the initial value problem for the nonlinear differential equations can be solved exactly. For the $\mathrm{KdV}$ equation this method was expressed in general form by Lax [73].

The essence of the application of the IST is as follows. The equation of interest for study (in our case VPE (50)) is written as the compatibility condition for two linear equations. These 
equations, (194) and (195), will be derived below. Then $W(X, 0)$ is mapped into the scattering data $S(0)$ for (194). It is important that since the variable $W(X, T)$ contained in the spectral equation (194) evolves according to (50), the spectrum $\lambda$ always retains constant values. The time evolution of $S(T)$ is simple and linear. From a knowledge of $S(T)$, we reconstruct $W(X, T)$.

8.1. Formulation of the Inverse Scattering Eigenvalue Problem. Since we have obtained the $N$-loop soliton solution to VPE (50) by use of the Hirota method (see Sections 5.4-5.8), we can state that VPE (50) is integrable. The use of the IST is the most appropriate way of tackling the initial value problem. In order to apply the IST method, one first has to formulate the associated eigenvalue problem. This can be achieved by finding a Bäcklund transformation associated with the VPE. We have already shown in Section 7 that the Bäcklund transformation is one of the analytical tools for dealing with soliton problems. The main aim of Section 8 is to give the details of the IST method for solving the VPE, so first we will formulate the scattering problem.

Now we will show that the IST problem for the VPE in form (50) has a third-order eigenvalue problem that is similar to the one associated with a higher order $\mathrm{KdV}$ equation [67, 74], a Boussinesq equation [74-78], and a model equation for shallow water waves $[14,49]$.

Introducing the function

$$
\psi=\frac{f^{\prime}}{f},
$$

and taking into account (70), we find that (173) and (174) reduce to

$$
\begin{array}{r}
\psi_{X X X}+W_{X} \psi_{X}-\lambda \psi=0, \\
3 \psi_{X T}+\left(1+W_{T}\right) \psi+\mu \psi_{X}=0,
\end{array}
$$

respectively, where we have used results similar to (X.1)-(X.3) in [14].

From (194) and (195) it can be shown that

$$
\begin{aligned}
& 3 \lambda \psi_{T}+\left(1+W_{T}\right) \psi_{X X}-W_{X T} \psi_{X} \\
& \quad+\left[W_{X X T}+\left(1+W_{T}\right) W_{X}+\mu \lambda\right] \psi=0, \\
& {\left[W_{X X T}+\left(1+W_{T}\right) W_{X}\right]_{X} \psi+\left(3 \psi_{T}+\mu \psi\right) \lambda_{X}=0 .}
\end{aligned}
$$

In view of (50), (196) becomes

$$
3 \lambda \psi_{T}+\left(1+W_{T}\right) \psi_{X X}-W_{X T} \psi_{X}+\lambda \mu \psi=0,
$$

and (197) implies that $\lambda_{X}=0$ so the spectrum $\lambda$ of (194) remains constant. Constant $\lambda$ is what is required in the IST problem. Equation (197) yields the equation $W_{X X T}+(1+$ $\left.W_{T}\right) W_{X}=h(T)$, where $h(T)$ is an arbitrary function of $T$. Now, according to (209) and (221), the inverse scattering method restricts the solutions to those that vanish as $|X| \rightarrow$ $\infty$, so $h(T)$ is to be identically zero. Thus the pair of (194) and
(195) or (194) and (197) can be considered as the Lax pair for VPE (50).

Since (194) and (195) are alternative forms of (173) and (174), respectively, it follows that the pair of (194) and (195) is associated with VPE (50) considered here. Thus the IST problem is directly related to a spectral equation of third order, namely, (194). The inverse problem for certain thirdorder spectral equations has been considered by Kaup [74] and Caudrey [75, 76]. As expected, (194) and (195) are similar to, but cannot be transformed into, the corresponding equations for the Hirota-Satsuma equation (HSE) (see Equations (A8a) and (A8b) in [79]). Clarkson and Mansfield [80] note that the scattering problem for the HSE is similar to that for the Boussinesq equation which has been studied comprehensively by Deift et al. [78].

After the Lax pair for the VPE was derived in [43], in [81] the Lax pair was written in its original variables as a zero curvature condition. Moreover, in [81] Hone and Wang have shown that there is a subtle connection between the SawadaKotera hierarchy and the VE, between the Degasperis-Procesi equation (DPE) and the VE (see also $[82,83]$ ), and between the Lax pairs of the DPE and the VE. For the Cauchy problem at long-time, the IST approach presents throughout a Riemann-Hilbert problem $[84,85]$ in original (physical) independent variables for the VE in [85].

8.2. Example of the Use of the IST Method to Find the OneSoliton Solution. Consider the one-soliton solution of the VPE obtained in Section 5.5, but by application of the IST method. Let the initial perturbation be

$$
W(X, 0)=6 k(1+\tanh (\eta)), \quad \eta=k X+\alpha .
$$

For convenience we introduce new notations $\xi_{1}$ and $\beta_{1}$ instead of parameters $k$ and $\alpha$ by

$$
\begin{aligned}
& k=\frac{\sqrt{3}}{2} \xi_{1}, \\
& \alpha=\frac{1}{2} \ln \left(\frac{\beta_{1}}{2 \sqrt{3} \xi_{1}}\right) ;
\end{aligned}
$$

then

$$
W(X, 0)
$$

$$
=6 \sqrt{3} \xi_{1} \frac{\partial}{\partial X} \ln \left[1+\frac{\beta_{1}}{2 \sqrt{3} \xi_{1}} \exp \left(\sqrt{3} \xi_{1} X\right)\right]
$$

is the initial condition for the VPE.

The first step in the IST method is to solve spectral equation (194) with spectral parameter $\lambda$ for the given initial condition $W(X, 0)$. In our example it is (201). The solution is studied over the complex $\zeta$-plane, where $\zeta^{3}=\lambda$. One can verify by direct substitution of (202) in (194) that the 
solution $\psi(X, 0 ; \zeta)$ of the linear ODE (194), normalized so that $\psi(X, 0 ; \zeta) \exp (-\zeta X) \rightarrow 1$ at $X \rightarrow-\infty$, is given by

$$
\begin{aligned}
& \psi(X, 0 ; \zeta) \exp (-\zeta X)=1 \\
& -\frac{\beta_{1} \exp \left(\sqrt{3} \xi_{1} X\right)}{1+\beta_{1}\left(\exp \left(\sqrt{3} \xi_{1} X\right) / 2 \sqrt{3} \xi_{1}\right)}\left[\frac{\omega_{2}}{i \omega_{2} \xi_{1}-\zeta}\right. \\
& \left.\quad+\frac{\omega_{3}}{-i \omega_{3} \xi_{1}-\zeta}\right]
\end{aligned}
$$

where $\omega_{j}=e^{i 2 \pi(j-1) / 3}$ are the cube of roots of $1(j=1,2,3)$. The constants $\beta_{1}$ and $\xi_{1}$, as we will show, are associated with the local spectral data.

The second step in the IST method is to obtain the evolution of $\beta_{1}$ and $\xi_{1}$. The time-dependence of the solution $\psi(X, T)$ is described by (195). Analyzing (195), we may assume that

$$
\begin{aligned}
& \xi_{1}(T)=\xi_{1}(0)=\text { const., } \\
& \beta_{1}(T)=\beta_{1}(0) \exp \left(-\frac{1}{\sqrt{3} \xi_{1}} T\right) .
\end{aligned}
$$

Below, the assumption of these relationships will be justified. Indeed, we know that the spectrum $\lambda$ in (194) remains constant if $W(X, T)$ evolves according to (50). Therefore, as will be proved, the spectrum data evolve as in (219). In notations (226) and (227), from (219) we obtain relations (203).

The final step in IST method is to select the solution $W(X, T)$ from (202) with $\xi_{1}(T)$ and $\beta_{1}(T)$ as in (203). According to Equation (2.7) in [74] we expand $\psi(X, T ; \zeta)$ as an asymptotic series in $\zeta^{-1}$ to obtain

$$
\begin{aligned}
\psi & (X, 0 ; \zeta) \exp (-\zeta X) \\
& =1-\frac{1}{3 \zeta}[W(X)-W(-\infty)]+O\left(\zeta^{-2}\right)
\end{aligned}
$$

that is, $W(X)-W(-\infty)=\lim _{\zeta \rightarrow \infty}[3 \zeta(1-\psi \exp (-\zeta X))]$. Taking into account the functional dependence (203), we find the required one-soliton solution of the VPE in form

$$
\begin{aligned}
& W(X, T)=6 \sqrt{3} \xi_{1} \frac{\partial}{\partial X} \\
& \cdot \ln \left[1+\frac{\beta_{1}}{2 \sqrt{3}} \exp \left(\sqrt{3} \xi_{1} X-\frac{1}{\sqrt{3} \xi_{1}} T\right)\right] \\
& + \text { const. }
\end{aligned}
$$

Thus, for the example of the one-soliton solution, we have demonstrated the IST method.

8.3. The Direct Spectral Problem. Let us consider the principal aspects of the inverse scattering transform problem for a third-order equation. The inverse problem for certain thirdorder spectral equations has been considered by Kaup [74] and Caudrey $[75,76]$. The time evolution of $\psi$ is determined from (195) or (198).
Following the method described by Caudrey [75], spectral equation (194) can be rewritten

$$
\frac{\partial}{\partial X} \psi=[\mathbf{A}(\zeta)+\mathbf{B}(X, \zeta)] \cdot \psi
$$

with

$$
\begin{aligned}
& \psi=\left(\begin{array}{c}
\psi \\
\psi_{X} \\
\psi_{X X}
\end{array}\right), \\
& \mathbf{A}=\left(\begin{array}{lll}
0 & 1 & 0 \\
0 & 0 & 1 \\
\lambda & 0 & 0
\end{array}\right), \\
& \mathbf{B}=\left(\begin{array}{lll}
0 & 0 & 0 \\
0 & 0 & 0 \\
0 & -W_{X} & 0
\end{array}\right) .
\end{aligned}
$$

The matrix $\mathbf{A}$ has eigenvalues $\lambda_{j}(\zeta)$ and left- and righteigenvectors $\widetilde{\mathbf{v}}_{j}(\zeta)$ and $\mathbf{v}_{j}(\zeta)$, respectively. These quantities are defined through a spectral parameter $\lambda$ as

$$
\begin{aligned}
& \lambda_{j}(\zeta)=\omega_{j} \zeta, \\
& \lambda_{j}^{3}(\zeta)=\lambda, \\
& \mathbf{v}_{j}(\zeta)=\left(\begin{array}{c}
1 \\
\lambda_{j}(\zeta) \\
\lambda_{j}^{2}(\zeta)
\end{array}\right), \\
& \widetilde{\mathbf{v}}_{j}(\zeta)=\left(\begin{array}{lll}
\lambda_{j}^{2}(\zeta) & \lambda_{j}(\zeta) & 1
\end{array}\right),
\end{aligned}
$$

where, as previously, $\omega_{j}=e^{2 \pi i(j-1) / 3}$ are the cube roots of 1 $(j=1,2,3)$. Obviously $\lambda_{j}(\zeta)$ are distinct and they and $\widetilde{\mathbf{v}}_{j}(\zeta)$ and $\mathbf{v}_{j}(\zeta)$ are analytic throughout the complex $\zeta$-plane.

The solution of linear equation (194) (or equivalently (206)) has been obtained by Caudrey [75] in terms of Jost functions $\phi_{j}(X, \zeta)$ which have the asymptotic behaviour

$$
\begin{aligned}
\Phi_{j}(X, \zeta):=\exp \left\{-\lambda_{j}(\zeta) X\right\} \phi_{j}(X, \zeta) & \longrightarrow \mathbf{v}_{j}(\zeta) \\
& \text { as } X \longrightarrow-\infty .
\end{aligned}
$$

Caudrey [75] showed how (206) can be solved by expressing it as a Fredholm integral equation.

The complex $\zeta$-plane is to be divided into regions such that, in the interior of each region, the order of the numbers $\operatorname{Re}\left(\lambda_{i}(\zeta)\right)$ is fixed. As we pass from one region to another this order changes and hence, on a boundary between two regions, $\operatorname{Re}\left(\lambda_{i}(\zeta)\right)=\operatorname{Re}\left(\lambda_{j}(\zeta)\right)$ for at least one pair $i \neq j$. The Jost function $\phi_{j}$ is regular throughout the complex $\zeta$-plane apart from poles and finite singularities on the boundaries between the regions. At any point in the interior of any region of the complex $\zeta$-plane, the solution of (206) is obtained by the relation (2.12) from [75]. It is the direct spectral problem. 
8.4. The Spectral Data. The information about the singularities of the Jost functions $\phi_{j}(X, \zeta)$ resides in the spectral data. First let us consider the poles. It is assumed that a pole $\zeta_{i}^{(k)}$ in $\phi_{i}(X, \zeta)$ is simple, does not coincide with a pole of $\phi_{j}(X, \zeta)$, $j \neq i$, and does not lie on a boundary between two regions. Then, as proven in [75], the residue is

$$
\operatorname{Res} \phi_{i}\left(X, \zeta_{i}^{(k)}\right)=\sum_{\substack{j=1 \\ j \neq i}}^{n} \gamma_{i j}^{(k)} \phi_{j}\left(X, \zeta_{i}^{(k)}\right)
$$

and it can be found because we know solution (194) in any regular regions from solving the direct problem (see Section 8.3). Note that, for $\phi_{j}\left(X, \zeta_{i}^{(k)}\right)$, the point $\zeta_{i}^{(k)}$ lies in the interior of a regular region. The quantities $\zeta_{i}^{(k)}$ and $\gamma_{i j}^{(k)}$ constitute the discrete part of the spectral data.

Now we consider the singularities on the boundaries between regions. However, in order to simplify matters, we first make some observations. The solution of the spectral problem can be facilitated by using various symmetry properties. In view of (194), we need only to consider the first elements of

$$
\phi_{i}(X, \zeta)=\left(\begin{array}{c}
\phi_{i}(X, \zeta) \\
\phi_{i}(X, \zeta)_{X} \\
\phi_{i}(X, \zeta)_{X X}
\end{array}\right)
$$

while the symmetry

$$
\phi_{1}\left(X, \frac{\zeta}{\omega_{1}}\right)=\phi_{2}\left(X, \frac{\zeta}{\omega_{2}}\right)=\phi_{3}\left(X, \frac{\zeta}{\omega_{3}}\right)
$$

means we need only to consider $\phi_{1}(X, \zeta)$. In our case, for $\phi_{1}(X, \zeta)$, the complex $\zeta$-plane is divided into four regions by two lines (see Figure 8 ) given by

(i) $\zeta^{\prime}=\omega_{2} \xi$, where $\operatorname{Re}\left(\lambda_{1}(\zeta)\right)=\operatorname{Re}\left(\lambda_{2}(\zeta)\right)$,

(ii) $\zeta^{\prime}=-\omega_{3} \xi$, where $\operatorname{Re}\left(\lambda_{1}(\zeta)\right)=\operatorname{Re}\left(\lambda_{3}(\zeta)\right)$,

where $\xi$ is real (see Figure 8 ). The singularity of $\phi_{1}(X, \zeta)$ can appear only on these boundaries between the regular regions on the $\zeta$-plane and it is characterized by functions $Q_{1 j}\left(\zeta^{\prime}\right)$ at each fixed $j \neq 1$. We denote the limit of a quantity, as the boundary is approached, by the superfix \pm according to the sign of $\operatorname{Re}\left(\lambda_{1}(\zeta)-\lambda_{j}(\zeta)\right)$ (see Figure 8 ).

In [75] (see Equation (3.14) there) the jump of $\phi_{1}(X, \zeta)$ on the boundaries is calculated as

$$
\phi_{1}^{+}(X, \zeta)-\phi_{1}^{-}(X, \zeta)=\sum_{j=2}^{3} Q_{1 j}(\zeta) \phi_{j}^{-}(X, \zeta),
$$

where, from (213), the sum is over the lines $\zeta^{\prime}=\omega_{2} \xi$ and $\zeta^{\prime}=-\omega_{3} \xi$ given by

(i) $\zeta^{\prime}=\omega_{2} \xi$, with $Q_{12}^{(1)}\left(\zeta^{\prime}\right) \neq 0, Q_{13}^{(1)}\left(\zeta^{\prime}\right) \equiv 0$,

(ii) $\zeta^{\prime}=-\omega_{3} \xi$, with $Q_{12}^{(2)}\left(\zeta^{\prime}\right) \equiv 0, Q_{13}^{(2)}\left(\zeta^{\prime}\right) \neq 0$.

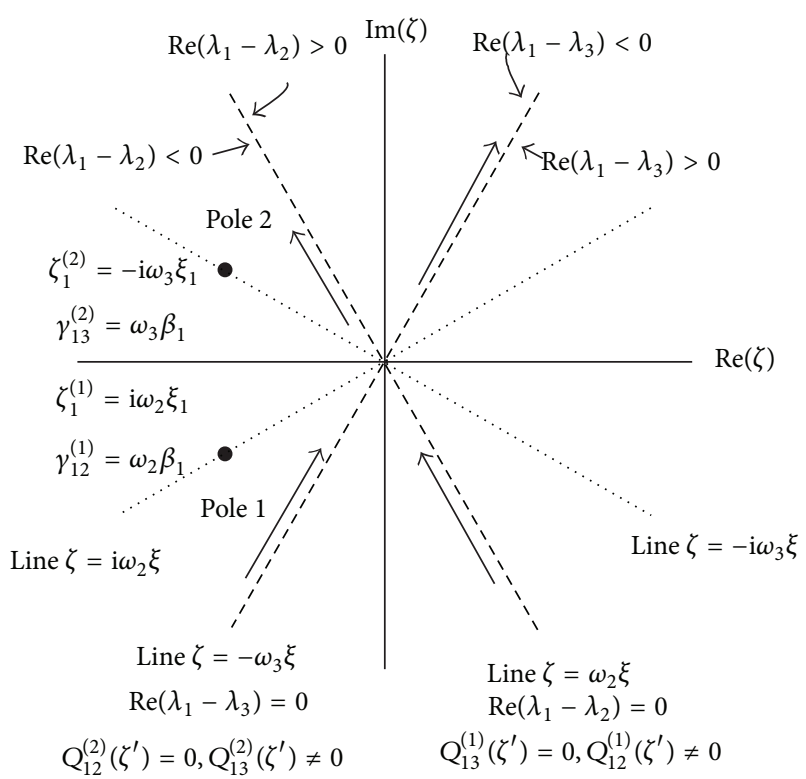

FIGURE 8: The regular regions for Jost functions $\phi_{1}(X, \zeta)$ in the complex $\zeta$-plane. The dashed lines determine the boundaries between regular regions. These lines are lines where the singularity functions $Q_{1 j}\left(\zeta^{\prime}\right)$ are given. The dotted lines are the lines where the poles appear.

The singularity functions $Q_{1 j}\left(\zeta^{\prime}\right)$ are determined by $W(X, 0)$ through the matrix $\mathbf{B}(X, \zeta)$ (207) (see Equation (3.13) in [75])

$$
\begin{aligned}
& Q_{1 j}(\zeta)=\frac{1}{\widetilde{\mathbf{v}}_{j}(\zeta) \cdot \mathbf{v}_{j}(\zeta)} \widetilde{\mathbf{v}}_{j}(\zeta) \\
& \quad \cdot \int_{-\infty}^{\infty} \exp \left[\left(\lambda_{1}(\zeta)-\lambda_{j}(\zeta)\right) z\right] \mathbf{B}(z, \zeta)
\end{aligned}
$$

$$
\cdot \phi_{1}^{-}(X, \zeta) d z
$$

The quantities $Q_{1 j}\left(\zeta^{\prime}\right)$ along all the boundaries constitute the continuum part of the spectral data.

Thus, the spectral data are

$$
S=\left\{\zeta_{1}^{(k)}, \gamma_{1 j}^{(k)}, Q_{1 j}\left(\zeta^{\prime}\right) ; j=2,3, k=1,2, \ldots, m\right\}
$$

One of the important features which is to be noted for the IST method is as follows. After the spectral data have been found from $\mathbf{B}(X, 0 ; \zeta)$, that is, at initial time, we need to seek the time evolution of the spectral data from (195). Analyzing (195) at $X \rightarrow \infty$ together with (209)

$$
\phi_{i}(X, T, \zeta)=\exp \left[-\left(3 \lambda_{i}(\zeta)\right)^{-1} T\right] \phi_{i}(X, 0, \zeta)
$$


the $T$-dependence is revealed as

$$
\begin{aligned}
& \zeta_{j}^{(k)}(T)=\zeta_{j}^{(k)}(0), \\
& \gamma_{1 j}^{(k)}(T)=\gamma_{1 j}^{(k)}(0) \\
& \cdot \exp \left\{\left[-\left(3 \lambda_{j}\left(\zeta_{1}^{(k)}\right)\right)^{-1}+\left(3 \lambda_{1}\left(\zeta_{1}^{(k)}\right)\right)^{-1}\right] T\right\}, \\
& Q_{1 j}\left(T ; \zeta^{\prime}\right)=Q_{1 j}\left(0 ; \zeta^{\prime}\right) \\
& \cdot \exp \left\{\left[-\left(3 \lambda_{j}\left(\zeta^{\prime}\right)\right)^{-1}+\left(3 \lambda_{1}\left(\zeta^{\prime}\right)\right)^{-1}\right] T\right\} .
\end{aligned}
$$

The final step in the application of the IST method is to reconstruct $\mathbf{B}(X, T ; \zeta)$ from the evaluated spectral data. In the next section, we show how to do this.

8.5. The Inverse Spectral Problem. The final procedure in IST method is that of the reconstruction of the matrix $\mathbf{B}(X, T ; \zeta)$ and $W(X, T)$ from the spectral data $S$.

The spectral data define $\Phi_{1}(X, \zeta)$ uniquely in the form (see Equation (6.20) in [75])

$$
\begin{aligned}
\Phi_{1}(X, T ; \zeta)=1-\sum_{k=1}^{K} \sum_{j=2}^{3} \gamma_{1 j}^{(k)}(T) \\
\cdot \frac{\exp \left\{\left[\lambda_{j}\left(\zeta_{1}^{(k)}\right)-\lambda_{1}\left(\zeta_{1}^{(k)}\right)\right] X\right\}}{\lambda_{1}\left(\zeta_{1}^{(k)}\right)-\lambda_{1}(\zeta)} \Phi_{1}\left(X, T ; \omega_{j} \zeta_{1}^{(k)}\right) \\
+\frac{1}{2 \pi i} \int \sum_{j=2}^{3} Q_{1 j}\left(T ; \zeta^{\prime}\right) \\
\quad \cdot \frac{\exp \left\{\left[\lambda_{j}\left(\zeta^{\prime}\right)-\lambda_{1}\left(\zeta^{\prime}\right)\right] X\right\}}{\zeta^{\prime}-\zeta} \Phi_{1}^{-}(X, T \\
\left.\omega_{j} \zeta^{\prime}\right) d \zeta^{\prime} .
\end{aligned}
$$

Equation (220) contains the spectral data, namely, $K$ poles with the quantities $\gamma_{1 j}^{(k)}$ for the bound state spectrum as well as the functions $Q_{1 j}\left(\zeta^{\prime}\right)$ given along all the boundaries of regular regions for the continuous spectrum. The integral in (220) is along all the boundaries (see the dashed lines in Figure 8). The direction of integration is taken so that the side chosen to be $\operatorname{Re}\left(\lambda_{1}(\zeta)-\lambda_{j}(\zeta)\right)<0$ is shown by the arrows in Figure 8 (for the lines (213), $\xi$ sweeps from $-\infty$ to $+\infty$ ).

It is necessary to note that we should carry out the integration along the lines $\omega_{2}(\xi+i \varepsilon)$ and $-\omega_{3}(\xi+i \varepsilon)$ with $\varepsilon>0$. In this case condition (209) is satisfied. Passing to the limit $\varepsilon \rightarrow 0$ we can obtain the solution which does not satisfy condition (209) (see Section 11.1.1). However, for any finite $\varepsilon>0$, the restricted region on $X$ can be determined where the solution associated with a finite $\varepsilon>0$ (for which condition (209) is valid) and the solution associated with $\varepsilon=0$ are sufficiently close to each other. In this sense, taking the integration at $\varepsilon=0$, we remain within the inverse scattering theory [75], and so condition (209) can be omitted. The solution obtained at $\varepsilon=0$ can be extended to sufficiently large finite $X$. Thus, we will interpret the solution obtained at $\varepsilon=0$ as the solution of VPE (50) which is valid for arbitrary but finite $X$.

By choosing appropriate values for $\zeta$, the left-hand side in (220) can be $\Phi_{1}\left(X, T ; \omega_{j} \zeta_{1}^{(k)}\right)$, or by allowing $\zeta$ to approach the boundaries from the appropriate sides, the left-hand side can be $\Phi_{1}^{-}\left(X, T ; \omega_{j} \zeta^{\prime}\right)$. We acquire a set of linear matrix/Fredholm equations in the unknowns $\Phi_{1}\left(X, T ; \omega_{j} \zeta_{1}^{(k)}\right)$ and $\Phi_{1}^{-}\left(X, T ; \omega_{j} \zeta^{\prime}\right)$. The solution of this equation system enables one to define $\Phi_{1}(X, T ; \zeta)$ from (220).

By knowing $\Phi_{1}(X, T ; \zeta)$, we can take extra information into account, namely, that the expansion of $\Phi_{1}(X, T ; \zeta)$ as an asymptotic series in $\lambda_{1}^{-1}(\zeta)$ connects with $W(X, T)$ as follows (cf. Equation (2.7) in [74]):

$$
\begin{aligned}
\Phi_{1}(X, T ; \zeta)= & 1-\frac{1}{3 \lambda_{1}(\zeta)}[W(X, T)-W(-\infty)] \\
& +O\left(\lambda_{1}^{-2}(\zeta)\right)
\end{aligned}
$$

Consequently, the solution $W(X, T)$ and the matrix $\mathbf{B}(X, T ; \zeta)$ can be reconstructed from the spectral data.

Sections 9-11 show how the IST method can be applied to the VPE.

\section{The N-Soliton Solution}

In this section the procedure for finding the exact $N$-soliton solution of the VPE via the inverse scattering method will be described [43, 61-63]. To do this we consider (220) with $Q_{1 j}(\zeta) \equiv 0$. Then there is only the bound state spectrum which is associated with the soliton solutions.

Let the bound state spectrum be defined by $K$ poles. Relation (220) is reduced to the form

$$
\begin{aligned}
& \Phi_{1}(X, T ; \zeta)=1-\sum_{k=1}^{K} \sum_{j=2}^{3} \gamma_{1 j}^{(k)}(T) \\
& \quad \cdot \frac{\exp \left\{\left[\lambda_{j}\left(\zeta_{1}^{(k)}\right)-\lambda_{1}\left(\zeta_{1}^{(k)}\right)\right] X\right\}}{\lambda_{1}\left(\zeta_{1}^{(k)}\right)-\lambda_{1}(\zeta)} \Phi_{1}(X, T ; \\
& \left.\omega_{j} \zeta_{1}^{(k)}\right) .
\end{aligned}
$$

Equation (222) involves the spectral data, namely, the poles $\zeta_{1}^{(k)}$ and the quantities $\gamma_{1 j}^{(k)}$. First we will prove that $\operatorname{Re} \lambda=0$ for compact support. From (194) we have

$$
\left(\psi_{X}\right)_{X X X}+\left(U \psi_{X}\right)_{X}-\lambda \psi_{X}=0
$$

and together with (194) this enables us to write

$$
\begin{aligned}
& \frac{\partial}{\partial X}\left(\frac{\partial^{2}}{\partial X^{2}} \psi_{X} \psi^{*}-3 \psi_{X X} \psi_{X}^{*}+U \psi_{X} \psi^{*}\right) \\
& -2 \operatorname{Re} \lambda \psi_{X} \psi^{*}=0 .
\end{aligned}
$$

Integrating (224) over all values of $X$, we obtain that, for compact support, $\operatorname{Re} \lambda=0$ since, in the general case, $\int_{-\infty}^{\infty} \psi_{X} \psi^{*} d X \neq 0$ 
As follows from (2.12), (2.13), (2.36), and (2.37) of [74], $\psi_{X}(\zeta)$ is related to the adjoint states $\psi_{X}^{A}(-\zeta)$. In the usual manner, using the adjoint states and Equation (14) from [76] and Equation (2.37) from [74], one can obtain

$$
\begin{aligned}
& \phi_{1 X}(X, \zeta)=\frac{i}{\sqrt{3}}\left[\phi_{1 X}\left(X,-\omega_{2} \zeta\right) \phi_{1}\left(X,-\omega_{3} \zeta\right)\right. \\
& \left.-\phi_{1 X}\left(X,-\omega_{3} \zeta\right) \phi_{1}\left(X,-\omega_{2} \zeta\right)\right] .
\end{aligned}
$$

It is easily seen that if $\zeta_{1}^{(1)}$ is a pole of $\phi_{1}(X, \zeta)$, then there is a pole either at $\zeta_{1}^{(2)}=-\omega_{2} \zeta_{1}^{(1)}$ (if $\phi_{1}\left(X,-\omega_{2} \zeta\right)$ has a pole) or at $\zeta_{1}^{(2)}=-\omega_{3} \zeta_{1}^{(1)}$ (if $\phi_{1}\left(X,-\omega_{3} \zeta\right)$ has a pole). For definiteness let $\zeta_{1}^{(2)}=-\omega_{2} \zeta_{1}^{(1)}$. Then, as follows from (225), $-\omega_{3} \zeta_{1}^{(2)}$ should be a pole. However, this pole coincides with the pole $\zeta_{1}^{(1)}$, since $-\omega_{3} \zeta_{1}^{(2)}=-\omega_{3}\left(-\omega_{2}\right) \zeta_{1}^{(1)}=\zeta_{1}^{(1)}$. Hence the poles appear in pairs, $\zeta_{1}^{(2 n-1)}$ and $\zeta_{1}^{(2 n)}$, under the condition $\zeta_{1}^{(2 n)} / \zeta_{1}^{(2 n-1)}=-\omega_{2}$, where $n$ is the pair number.

Let us consider $N$ pairs of poles; that is, in all there are $K=2 N$ poles over which the sum is taken in (225). For the pair $n(n=1,2, \ldots, N)$ we have the properties

$$
\begin{aligned}
& \text { (i) } \zeta_{1}^{(2 n-1)}=i \omega_{2} \xi_{n} \\
& \text { (ii) } \zeta_{1}^{(2 n)}=-i \omega_{3} \xi_{n}
\end{aligned}
$$

Since $U$ is real and $\lambda$ is imaginary, $\xi_{k}$ is real. Relationships (226) are in line with condition (2.33) from [74]. These relationships are also similar to Equations (6.24) and (6.25) in [75], while $\gamma_{1 j}^{(k)}$ turns out to be different from $\widetilde{\gamma}_{1 j}^{(k)}$ for the Boussinesq equation (see Equations (6.24) and (6.25) in [75]). Indeed, by considering (225) in the vicinity of the first pole $\zeta_{1}^{(2 n-1)}$ of the pair $n$ and using relation (222), one can obtain a relation between $\gamma_{12}^{(k)}$ and $\gamma_{13}^{(k)}$. In this case the functions $\phi_{1, X}(X, \zeta), \phi_{1}\left(X,-\omega_{2} \zeta\right)$, and $\phi_{1, X}\left(X,-\omega_{2} \zeta\right)$ also have poles here, while the functions $\phi_{1}\left(X,-\omega_{3} \zeta\right)$ and $\phi_{1, X}\left(X,-\omega_{3} \zeta\right)$ do not have poles here. Substituting $\phi_{1}(X, \zeta)$ in form (222) into (225) and letting $X \rightarrow-\infty$, we have the ratio $\gamma_{13}^{(2 n)} / \gamma_{12}^{(2 n-1)}=$ $\omega_{2}$ and $\gamma_{12}^{(2 n)}=\gamma_{13}^{(2 n-1)}=0$. Therefore the properties of $\gamma_{i j}^{(k)}$ should be defined by the relationships

$$
\begin{array}{ll}
\text { (i) } \gamma_{12}^{(2 n-1)}=\omega_{2} \beta_{k}, & \gamma_{13}^{(2 n-1)}=0, \\
\text { (ii) } \gamma_{12}^{(2 n)}=0, & \gamma_{13}^{(2 n)}=\omega_{3} \beta_{k},
\end{array}
$$

where, as it will be proved below, $\beta_{k}$ is real when $U=W_{X}$ is real.

By defining

$$
\begin{aligned}
\Psi_{k} & (X, T) \\
& =\sum_{j=2}^{3} \gamma_{1 j}^{(k)}(T) \exp \left\{\lambda_{j}\left(\zeta_{1}^{(k)}\right) X\right\} \Phi_{1}\left(X, T ; \omega_{j} \zeta_{1}^{(k)}\right),
\end{aligned}
$$

we may rewrite relationship (222) as (see, for instance, Equations (6.33) and (6.34) in [75])

$$
\Phi_{1}(X, T ; \zeta)=1-\sum_{k=1}^{2 N} \frac{\exp \left\{-\lambda_{1}\left(\zeta_{1}^{(k)}\right) X\right\}}{\lambda_{1}\left(\zeta_{1}^{(k)}\right)-\lambda_{1}(\zeta)} \Psi_{k}(X, T) .
$$

From (221) and (229) it may be shown that (cf. Equation (6.38) in [75])

$$
\begin{aligned}
W & (X, T)-W(-\infty) \\
& =-3 \sum_{k=1}^{2 N} \exp \left\{-\lambda_{1}\left(\zeta_{1}^{(k)}\right) X\right\} \Psi_{k}(X, T) \\
& =3 \frac{\partial}{\partial X} \ln (\operatorname{det} M(X, T)) .
\end{aligned}
$$

The $2 N \times 2 N$ matrix $M(X, T)$ is defined as in relationship (6.36) in [75] by

$$
M_{k l}(X, T)=\delta_{k l}-\sum_{j=2}^{3} \gamma_{1 j}^{(k)}(0) \frac{\exp \left\{\left[-\left(3 \lambda_{j}\left(\zeta_{1}^{(k)}\right)\right)^{-1}+\left(3 \lambda_{1}\left(\zeta_{1}^{(k)}\right)\right)^{-1}\right] T+\left(\lambda_{j}\left(\zeta_{1}^{(k)}\right)-\lambda_{1}\left(\zeta_{1}^{(l)}\right)\right) X\right\}}{\lambda_{j}\left(\zeta_{1}^{(k)}\right)-\lambda_{1}\left(\zeta_{1}^{(l)}\right)}
$$

and $n=1,2, \ldots, N$,

$$
\begin{aligned}
\lambda_{1}\left(\zeta_{1}^{(2 n-1)}\right) & =i \omega_{2} \xi_{n}, \\
\lambda_{2}\left(\zeta_{1}^{(2 n-1)}\right) & =i \omega_{3} \xi_{n}, \\
\gamma_{12}^{(2 n-1)} & =\omega_{2} \beta_{n}, \\
\gamma_{13}^{(2 n-1)} & =0, \\
\lambda_{1}\left(\zeta_{1}^{(2 n)}\right) & =-i \omega_{3} \xi_{n}, \\
\lambda_{3}\left(\zeta_{1}^{(2 n)}\right) & =-i \omega_{2} \xi_{n},
\end{aligned}
$$

$$
\begin{aligned}
& \gamma_{12}^{(2 n)}=0, \\
& \gamma_{13}^{(2 n)}=\omega_{3} \beta_{n} .
\end{aligned}
$$

For the $N$-soliton solution there are $N$ arbitrary constants $\xi_{n}$ and $N$ arbitrary constants $\beta_{n}$.

The final result for the $N$-soliton solution of the VPE is defined by relationship (230) with (231).

9.1. Examples of One- and Two-Soliton Solutions. In order to obtain the one-soliton solution of VPE (50)

$$
W_{X X T}+\left(1+W_{T}\right) W_{X}=0,
$$


we need first to calculate the $2 \times 2$ matrix $M(X, T)$ according to (231) with $N=1$. We find that the matrix is

$$
\left(\begin{array}{cc}
1-\frac{\omega_{2} \beta_{1}}{\sqrt{3} \xi_{1}} \exp \left[\sqrt{3} \xi_{1} X-\left(\sqrt{3} \xi_{1}\right)^{-1} T\right] & \frac{i \omega_{3} \beta_{1}}{2 \xi_{1}} \exp \left[2 i \omega_{3} \xi_{1} X-\left(\sqrt{3} \xi_{1}\right)^{-1} T\right] \\
\frac{-i \omega_{2} \beta_{1}}{2 \xi_{1}} \exp \left[-2 i \omega_{2} \xi_{1} X-\left(\sqrt{3} \xi_{1}\right)^{-1} T\right] & 1-\frac{\omega_{3} \beta_{1}}{\sqrt{3} \xi_{1}} \exp \left[\sqrt{3} \xi_{1} X-\left(\sqrt{3} \xi_{1}\right)^{-1} T\right]
\end{array}\right)
$$

and its determinant is

$$
\begin{aligned}
& \operatorname{det} M(X, T) \\
& =\left\{1+\frac{\beta_{1}}{2 \sqrt{3} \xi_{1}} \exp \left[\sqrt{3} \xi_{1}\left(X-\frac{T}{3 \xi_{1}^{2}}\right)\right]\right\}^{2} .
\end{aligned}
$$

Consequently, from (230) we have the one-soliton solution of the VPE:

$$
\begin{aligned}
U(X, T) & =W_{X}(X, T) \\
& =\frac{9}{2} \xi_{1}^{2} \operatorname{sech}^{2}\left[\frac{\sqrt{3}}{2} \xi_{1}\left(X-\frac{T}{3 \xi_{1}^{2}}\right)+\alpha_{1}\right],
\end{aligned}
$$

where $\alpha_{1}=(1 / 2) \ln \left(\beta_{1} / 2 \sqrt{3} \xi_{1}\right)$ is an arbitrary constant. Since $U$ is real, it follows from (236) that $\beta_{1}$ is real. By writing $\sqrt{3} \xi_{1} / 2=k$ in (236), with the condition $\beta_{1} / \xi_{1}>0$ we recover the one-soliton solution as we found previously by Hirota's method (see Equation (3.4) in [36] and/or (89)).

Note that with $\beta_{1} / \xi_{1}<0$ we have the real solution in the form of the singular soliton (93) [58]. Analysis of the singular soliton solution is presented in Section 11.2 and Appendix B.

It is of interest to compare (236) with the solution of the fifth-order KdV-like equation discussed in [74]. Spectral equation (195) is the same as that given by (1.1) (with $R=0$ ) in [74], whereas the equation that governs the time-dependence of $\psi$, that is, (195), is different from (1.2) in [74]. Thus the $X$ dependence of (235) should agree with the $x$ dependence of the solution given by (3.30) in [74]. With the identification $U=5 Q, \xi_{1}=\eta$, this is indeed the case.

Let us now consider the two-soliton solution of the VPE. In this case $M(X, T)$ is a $4 \times 4$ matrix. We will not give the explicit form here, but we find that

$$
\operatorname{det} M(X, T)=\left(1+q_{1}^{2}+q_{2}^{2}+b^{2} q_{1}^{2} q_{2}^{2}\right)^{2}
$$

where

$$
\begin{aligned}
& q_{i}=\exp \left[\frac{\sqrt{3}}{2} \xi_{i}\left(X-\frac{T}{3 \xi_{i}^{2}}\right)+\alpha_{i}\right], \\
& b^{2}=\left(\frac{\xi_{2}-\xi_{1}}{\xi_{2}+\xi_{1}}\right)^{2} \frac{\xi_{1}^{2}+\xi_{2}^{2}-\xi_{1} \xi_{2}}{\xi_{1}^{2}+\xi_{2}^{2}+\xi_{1} \xi_{2}}
\end{aligned}
$$

and $\alpha_{i}=(1 / 2) \ln \left(\beta_{i} / 2 \sqrt{3} \xi_{i}\right)$ are arbitrary constants. The twosoliton solution to the VPE as found by the IST method is given by (230) together with (237). With the identification $\sqrt{3} \xi_{i} / 2=k_{i}(i=1,2)$ we recover the two-soliton solution as given by Hirotas method (see Equations (4.1)-(4.5) in [36] and/or (98)).

Finally we note that comparison of (230) with $W=$ $6(\ln f)_{X}$ from (70) shows that

$$
\ln (\operatorname{det} M(X, T))=2 \ln (f)
$$

so that $\operatorname{det} M(X, T)$ is a perfect square for arbitrary $N$.

\section{Accounting for the Continuum Part of Spectral Data}

Now, in addition to the bound state spectrum, we consider the continuous spectrum of the associated eigenvalue problem $[44-46,86]$; that is, assume that at least some of the functions $Q_{1 j}\left(\zeta^{\prime}\right)$ are nonzero. At each fixed $j \neq 1$ the functions $Q_{1 j}\left(\zeta^{\prime}\right)$ characterize the singularity of $\Phi_{1}(X, \zeta)$. As we have shown, this singularity can appear only on boundaries between the regular regions on the $\zeta$-plane, where the condition $\operatorname{Re}\left(\lambda_{1}\left(\zeta^{\prime}\right)-\lambda_{j}\left(\zeta^{\prime}\right)\right)=0$ constitutes these boundaries [75]. For $\operatorname{VPE}(50)$

$$
W_{X X T}+\left(1+W_{T}\right) W_{X}=0,
$$

as we know, the complex $\zeta$-plane is divided into four regions by two lines (215)

$$
\begin{aligned}
& \text { (i) } \zeta^{\prime}=\omega_{2} \xi, \quad \text { with } Q_{12}^{(1)}\left(\zeta^{\prime}\right) \neq 0, Q_{13}^{(1)}\left(\zeta^{\prime}\right) \equiv 0, \\
& \text { (ii) } \zeta^{\prime}=-\omega_{3} \xi, \quad \text { with } Q_{12}^{(2)}\left(\zeta^{\prime}\right) \equiv 0, Q_{13}^{(2)}\left(\zeta^{\prime}\right) \neq 0,
\end{aligned}
$$

where $\xi$ is real (see Figure 8) and sweeps from $-\infty$ to $+\infty$. 
Let us consider the singularity functions $Q_{1 j}\left(\zeta^{\prime}\right)$ on the boundaries, on which the Jost function $\phi_{1}(X, \zeta)$ is singular, in the form $(m=1,2, \ldots, M)$ :

$$
\begin{aligned}
& Q_{12}^{(1)}\left(\zeta^{\prime}\right)=-2 \pi i \sum_{m=1}^{M} q_{12}^{(2 m-1)} \delta\left(\zeta^{\prime}-\zeta_{2 n-1}^{\prime}\right), \\
& Q_{13}^{(1)}\left(\zeta^{\prime}\right)=-2 \pi i \sum_{m=1}^{M} q_{13}^{(2 m-1)} \delta\left(\zeta^{\prime}-\zeta_{2 n-1}^{\prime}\right) \equiv 0, \\
& \text { on the line } \zeta^{\prime}=\omega_{2} \xi, \\
& Q_{12}^{(2)}\left(\zeta^{\prime}\right)=-2 \pi i \sum_{m=1}^{M} q_{12}^{(2 m)} \delta\left(\zeta^{\prime}-\zeta_{2 n}^{\prime}\right) \equiv 0, \\
& Q_{13}^{(2)}\left(\zeta^{\prime}\right)=-2 \pi i \sum_{m=1}^{M} q_{13}^{(2 m)} \delta\left(\zeta^{\prime}-\zeta_{2 n}^{\prime}\right),
\end{aligned}
$$

$$
\text { on the line } \zeta^{\prime}=-\omega_{3} \xi \text {. }
$$

For the singularity functions (242) and for $N$ pairs of poles, relationship (220) is reduced to the form (provisionally the time-dependence is not written)

$$
\begin{gathered}
\Phi_{1}(X, \zeta)=1-\sum_{k=1}^{2 N} \sum_{j=2}^{3} \gamma_{1 j}^{(k)} \\
\cdot \frac{\exp \left\{\left[\lambda_{j}\left(\zeta_{1}^{(k)}\right)-\lambda_{1}\left(\zeta_{1}^{(k)}\right)\right] X\right\}}{\lambda_{1}\left(\zeta_{1}^{(k)}\right)-\lambda_{1}(\zeta)} \Phi_{1}\left(X, \omega_{j} \zeta_{1}^{(k)}\right) \\
\quad-\sum_{l=1}^{2 M} \sum_{j=2}^{3} q_{1 j}^{(l)} \\
. \frac{\exp \left\{\left[\lambda_{j}\left(\zeta_{l}^{\prime}\right)-\lambda_{1}\left(\zeta_{l}^{\prime}\right)\right] X\right\}}{\zeta_{l}^{\prime}-\zeta} \Phi_{1}\left(X, \omega_{j} \zeta_{l}^{\prime}\right) .
\end{gathered}
$$

In Section 9 (see [43] too) it is proved that the poles appear in pairs only $\zeta_{1}^{(2 n-1)}=i \omega_{2} \xi_{n}$ and $\zeta_{1}^{(2 n)}=-i \omega_{3} \xi_{n}$, under the conditions $\gamma_{12}^{(2 n-1)}=\omega_{2} \beta_{n}, \gamma_{13}^{(2 n-1)}=0, \gamma_{12}^{(2 n)}=0, \gamma_{13}^{(2 n)}=$ $\omega_{3} \beta_{n}(n=1,2, \ldots, N)$. If we consider both the bound state spectrum and the continuous spectrum, the constants $\beta_{n}$ are complex values in the general case. The restrictions on $\beta_{n}$ for real solutions $U=W_{X}$ follow from a separate problem which will be analyzed in Section 11.

As follows from relationships (225) and (243), the singularities in form (242) appear in pairs $\zeta_{2 m-1}^{\prime}=\omega_{2} \xi_{m}$ and $\zeta_{2 m}^{\prime}=-\omega_{3} \xi_{m}$. From (225), on considering the limits $\zeta \rightarrow \zeta_{l}^{\prime}$ and $X \rightarrow-\infty$, it immediately follows that

$$
q_{12}^{(2 m-1)} \omega_{2}=q_{13}^{(2 m)} \text { for } m=1,2, \ldots, M .
$$

Insofar as we have $2 N$ poles and $2 M$ coefficients $q_{12}^{(2 m-1)}$ and $q_{13}^{(2 m)}$ in adopted specifications (242) of the singularity functions $Q_{1 j}\left(\zeta^{\prime}\right)$, it is convenient to introduce the notation

$$
\begin{aligned}
& \mu_{j i}=\left\{\begin{array}{l}
\lambda_{j}\left(\zeta_{1}^{(i)}\right) \\
\lambda_{j}\left(\zeta_{(i-K)}^{\prime}\right),
\end{array}\right. \\
& p_{1 j}^{(i)}= \begin{cases}\gamma_{1 j}^{(i)} & \text { at } i=1, \ldots, K \\
q_{1 j}^{(i-K)} & \text { at } i=K+1, \ldots, K+L,\end{cases}
\end{aligned}
$$

where $K=2 N$ and $L=2 M$. Then relationship (243) is rewritten as follows:

$$
\begin{aligned}
& \Phi_{1}(X, \zeta) \\
& \quad=1-\sum_{i=1}^{K+L} \sum_{j=2}^{3} p_{1 j}^{(i)} \frac{\exp \left[\left(\mu_{j i}-\mu_{1 i}\right) X\right]}{\mu_{1 i}-\zeta} \Phi_{1}\left(X, \mu_{j i}\right) .
\end{aligned}
$$

By defining

$$
\Psi_{i}(X)=\sum_{j=2}^{3} p_{1 j}^{(i)} \exp \left(\mu_{j i} X\right) \Phi_{1}\left(X, \mu_{j i}\right),
$$

we may rewrite relationship (246) as

$$
\Phi_{1}(X, \zeta)=1-\sum_{i=1}^{K+L} \frac{\exp \left(-\mu_{1 i} X\right)}{\mu_{1 i}-\zeta} \Psi_{i}(X)
$$

Taking into account (221), namely,

$$
\begin{aligned}
\Phi_{1}(X, \zeta)= & 1-\frac{1}{3 \lambda_{1}(\zeta)}[W(X)-W(-\infty)] \\
& +O\left(\lambda_{1}^{-2}(\zeta)\right)
\end{aligned}
$$

and (247) and (248), the following key relationship may be found (see also (230)):

$$
\begin{aligned}
W(X)-W(-\infty) & =-3 \sum_{k=1}^{L+M} \exp \left(-\mu_{j k} X\right) \Psi_{k}(X) \\
& =3 \frac{\partial}{\partial X} \ln (\operatorname{det} M(X)) .
\end{aligned}
$$

Here the matrix $M(X)$ is defined as follows:

$$
M_{i l}(X)=\delta_{i l}-\sum_{j=2}^{3} p_{1 j}^{(i)} \frac{\exp \left[\left(\mu_{j i}-\mu_{1 l}\right) X\right]}{\mu_{j i}-\mu_{1 l}} .
$$

Restoring the $T$-evolution in the relationships, the final result for the solution of the VPE, when we consider the spectral data from both the bound state spectrum and the continuous spectrum, is as follows:

$$
U(X, T)=W_{X}(X, T)=3 \frac{\partial^{2}}{\partial X^{2}} \ln (\operatorname{det} M(X, T)) .
$$


Here $M(X, T)$ is the $(K+L) \times(K+L)$ matrix given by

$$
\begin{aligned}
& M_{k l}(X, T)=\delta_{k l}-\sum_{j=2}^{3} p_{1 j}^{(k)} \\
& \cdot \frac{\exp \left\{\left(\mu_{j k}-\mu_{1 l}\right) X+\left[-\left(3 \mu_{j k}\right)^{-1}+\left(3 \mu_{1 k}\right)^{-1}\right] T\right\}}{\mu_{j k}-\mu_{1 l}},
\end{aligned}
$$

where, for $i \leq N$,

$$
\begin{aligned}
\mu_{1(2 i-1)} & =\lambda_{1}\left(\zeta_{1}^{(2 i-1)}\right)=i \omega_{2} \xi_{i}, \\
\mu_{2(2 i-1)} & =\lambda_{2}\left(\zeta_{1}^{(2 i-1)}\right)=i \omega_{3} \xi_{i} \\
p_{12}^{(2 i-1)} & =\gamma_{12}^{(2 i-1)}=\omega_{2} \beta_{i}, \\
p_{13}^{(2 i-1)} & =\gamma_{13}^{(2 i-1)}=0 \\
\mu_{1(2 i)} & =\lambda_{1}\left(\zeta_{1}^{(2 i)}\right)=-i \omega_{3} \xi_{i}, \\
\mu_{3(2 i)} & =\lambda_{3}\left(\zeta_{1}^{(2 i)}\right)=-i \omega_{2} \xi_{i}, \\
p_{12}^{(2 i)} & =\gamma_{12}^{(2 i)}=0 \\
p_{13}^{(2 i)} & =\gamma_{13}^{(2 i)}=\omega_{3} \beta_{i},
\end{aligned}
$$

and, for $N<i \leq N+M$,

$$
\begin{gathered}
\mu_{1(2 i-1)}=\lambda_{1}\left(\zeta_{2(i-M)-1}^{\prime}\right)=\omega_{2} \xi_{i}, \\
\mu_{2(2 i-1)}=\lambda_{2}\left(\zeta_{2(i-M)-1}^{\prime}\right)=\omega_{3} \xi_{i}, \\
p_{12}^{(2 i-1)}=q_{12}^{(2(i-M)-1)}=\omega_{2} \beta_{i}, \\
p_{13}^{(2 i-1)}=q_{13}^{(2(i-M)-1)}=0, \\
\mu_{1(2 i)}=\lambda_{1}\left(\zeta_{2(i-M)}^{\prime}\right)=-\omega_{3} \xi_{i}, \\
\mu_{3(2 i)}=\lambda_{3}\left(\zeta_{2(i-M)}^{\prime}\right)=-\omega_{2} \xi_{i}, \\
p_{12}^{(2 i)}=q_{12}^{(2(i-M))}=0, \\
p_{13}^{(2 i)}=q_{13}^{(2(i-M))}=\omega_{3} \beta_{i} .
\end{gathered}
$$

For solution (252) and (253) there are $(N+M)$ arbitrary constants $\xi_{i}$ and $(N+M)$ arbitrary constants $\beta_{i}$. The constants $\xi_{i}$ are real, while the constants $\beta_{i}$, in the general case, are complex.

As will be clear from the examples in Section 11, solution (252) and (253) includes $M$ discrete frequencies from the continuum part of the spectral data. For this reason, solution (252) and (253) without solitons (i.e., with $N=0$ ) will be referred to as an $M$-mode solution of the VPE. Evidently these discrete modes emanate from the special choice (242) of the singularity functions $Q_{1 j}\left(\zeta^{\prime}\right)$.

The solution obtained through matrix (253) is in general a complex function. Consequently, there is a problem in selecting the real solutions from the complex solutions. It turns out that we can obtain the real solutions by means of restriction of arbitrariness in the choice of the constants $\beta_{i}$. We have succeeded in finding these restrictions.

\section{Real Solutions for the VPE}

Now we select the real solutions $U=W_{X}$ from (252) and (253). We analyze a number of examples, as well as the general case, for the interaction of the solitons and multimode waves $[44-46,86]$. To obtain the solutions of the VPE, one has to calculate the determinant of matrix (253). Firstly, we present four results of such a calculation for $N+M \leq 4$. For convenience we will use the auxiliary function $F(X, T)$ given by the definition $F(X, T)=\sqrt{\operatorname{det} M(X, T)}$. In particular, from (253),

(1) for $N+M=1$ we have

$$
F=1+c_{1} q_{1}
$$

(2) for $N+M=2$ we have

$$
F=1+c_{1} q_{1}+c_{2} q_{2}+b_{12} c_{1} c_{2} q_{1} q_{2}
$$

(3) for $N+M=3$ we have

$$
\begin{aligned}
F= & 1+c_{1} q_{1}+c_{2} q_{2}+c_{3} q_{3}+b_{12} c_{1} c_{2} q_{1} q_{2} \\
& +b_{13} c_{1} c_{3} q_{1} q_{3}+b_{23} c_{2} c_{3} q_{2} q_{3} \\
& +b_{12} b_{13} b_{23} c_{1} c_{2} c_{3} q_{1} q_{2} q_{3}
\end{aligned}
$$

(4) for $N+M=4$ we have

$$
\begin{aligned}
F= & 1+c_{1} q_{1}+c_{2} q_{2}+c_{3} q_{3}+c_{4} q_{4}+b_{12} c_{1} c_{2} q_{1} q_{2} \\
& +b_{13} c_{1} c_{3} q_{1} q_{3}+b_{14} c_{1} c_{4} q_{1} q_{4}+b_{23} c_{2} c_{3} q_{2} q_{3} \\
& +b_{24} c_{2} c_{4} q_{2} q_{4}+b_{34} c_{3} c_{4} q_{3} q_{4} \\
& +b_{12} b_{13} b_{23} c_{1} c_{2} c_{3} q_{1} q_{2} q_{3}+b_{12} b_{14} b_{24} c_{1} c_{2} c_{4} q_{1} q_{2} q_{4} \\
& +b_{13} b_{14} b_{34} c_{1} c_{3} c_{4} q_{1} q_{3} q_{4}+b_{23} b_{24} b_{34} c_{2} c_{3} c_{4} q_{2} q_{3} q_{4} \\
& +b_{12} b_{13} b_{14} b_{23} b_{24} b_{34} c_{1} c_{2} c_{3} c_{4} q_{1} q_{2} q_{3} q_{4} .
\end{aligned}
$$

For $N+M>4$, the explicit expression for the function $F(X, T)$ can be obtained in a similar manner. It is helpful to present the quantities $c_{i}, q_{i}$, and $b_{i j}$ involved in formulas (256)-(259) separately for three distinct cases:

(1) The purely solitonic case $(i, j) \leq N$ has

$$
\begin{gathered}
q_{i}=\exp \left(2 \theta_{i}\right) \\
2 \theta_{i}=\sqrt{3} \xi_{i} X-\left(\sqrt{3} \xi_{i}\right)^{-1} T, c_{i}=\frac{\beta_{i}}{2 \sqrt{3} \xi_{i}}, \\
b_{i j}=\left(\frac{\xi_{i}-\xi_{j}}{\xi_{i}+\xi_{j}}\right)^{2} \frac{\xi_{i}^{2}+\xi_{j}^{2}-\xi_{i} \xi_{j}}{\xi_{i}^{2}+\xi_{j}^{2}+\xi_{i} \xi_{j}}, \quad b_{i j} \geq 0 .
\end{gathered}
$$


(2) The case of purely multimode waves $N<(i, j) \leq N+$ $M$ has

$q_{i}=\exp \left(2 \theta_{i}\right)$

$$
2 \theta_{i}=-i \sqrt{3} \xi_{i} X+\left(i \sqrt{3} \xi_{i}\right)^{-1} T, c_{i}=\frac{i \beta_{i}}{2 \sqrt{3} \xi_{i}},
$$

$b_{i j}=\left(\frac{\xi_{i}-\xi_{j}}{\xi_{i}+\xi_{j}}\right)^{2} \frac{\xi_{i}^{2}+\xi_{j}^{2}-\xi_{i} \xi_{j}}{\xi_{i}^{2}+\xi_{j}^{2}+\xi_{i} \xi_{j}}, \quad b_{i j} \geq 0$.

(3) The case of a combination of solitons $\left(i, i^{\prime}\right) \leq N$ and multimode waves $N<\left(j, j^{\prime}\right) \leq N+M$ has

$$
\begin{aligned}
& q_{i}= \exp \left(2 \theta_{i}\right) \\
& 2 \theta_{i}=\sqrt{3} \xi_{i} X-\left(\sqrt{3} \xi_{i}\right)^{-1} T, c_{i}=\frac{\beta_{i}}{2 \sqrt{3} \xi_{i}}, \\
& q_{j}=\exp \left(2 \theta_{j}\right) \\
& 2 \theta_{j}=-i \sqrt{3} \xi_{j} X+\left(i \sqrt{3} \xi_{j}\right)^{-1} T, c_{j}=\frac{i \beta_{j}}{2 \sqrt{3} \xi_{j}}, \\
& b_{i i^{\prime}}=\left(\frac{\xi_{i}-\xi_{i^{\prime}}}{\xi_{i}+\xi_{i^{\prime}}}\right)^{2} \frac{\xi_{i}^{2}+\xi_{i^{\prime}}^{2}-\xi_{i} \xi_{i^{\prime}}}{\xi_{i}^{2}+\xi_{i^{\prime}}^{2}+\xi_{i} \xi_{i^{\prime}}}, \quad 0 \leq b_{i i^{\prime}} \leq 1, \\
& b_{j j^{\prime}=}\left(\frac{\xi_{j}-\xi_{j^{\prime}}}{\xi_{j}+\xi_{j^{\prime}}}\right)^{2} \frac{\xi_{j}^{2}+\xi_{j^{\prime}}^{2}-\xi_{j} \xi_{j^{\prime}}}{\xi_{j}^{2}+\xi_{j^{\prime}}^{2}+\xi_{j} \xi_{j^{\prime}}}, \quad 0 \leq b_{j j^{\prime}} \leq 1, \\
& b_{i j}=\left(\frac{\xi_{i}+i \xi_{j}}{\xi_{i}-i \xi_{j}}\right)^{2} \frac{\xi_{i}^{2}-\xi_{j}^{2}+i \xi_{i} \xi_{j}}{\xi_{i}^{2}-\xi_{j}^{2}-i \xi_{i} \xi_{j}}, \quad\left|b_{i j}\right| \equiv 1 .
\end{aligned}
$$

With the above found representation of the auxiliary function $F(X, T)$ and taking into account key relationship (252), we can write the explicit solution to basic nonlinear evolution equation (50) in the following concise form:

$$
W(X, T)=6 \frac{\partial}{\partial X} \ln (F(X, T))+\text { const. }
$$

The function $F$ is complex-valued in the general case because the values of $\beta_{i}$ (and hence of $c_{i}$ ) are complex constants.

Since we are interested only in the real solution $W_{X}$ with real constants $\xi_{i}$, we need restrictions on the constants $c_{i}$ in (256)-(259).

11.1. The Solutions Associated with the Continuous Spectrum. We study the multimode solutions for $N=0$ and $M=$ $1,2,3,4$, while for $M \geq 5$ all formulas can easily be obtained by means of a generalization of these examples.

11.1.1. The One-Mode Solution. In order to obtain the onemode solution of VPE (50) we need first to calculate the $2 \times 2$ matrix $M(X, T)$ according to (253) with $N=0$ and $M=1$. For the matrix elements $M_{k l}(X, T)$ we have

$$
\begin{aligned}
& M_{11}(X, T) \\
& =1-\frac{i \omega_{2} \beta_{1}}{\sqrt{3} \xi_{1}} \exp \left[-i \sqrt{3} \xi_{1} X+\left(i \sqrt{3} \xi_{1}\right)^{-1} T\right] \\
& M_{12}(X, T)=-\frac{\omega_{3} \beta_{1}}{2 \xi_{1}} \exp \left[2 \omega_{3} \xi_{1} X+\left(i \sqrt{3} \xi_{1}\right)^{-1} T\right] \\
& M_{21}(X, T)=\frac{\omega_{2} \beta_{1}}{2 \xi_{1}} \exp \left[-2 \omega_{2} \xi_{1} X+\left(i \sqrt{3} \xi_{1}\right)^{-1} T\right] \\
& M_{22}(X, T) \\
& =1-\frac{i \omega_{3} \beta_{1}}{\sqrt{3} \xi_{1}} \exp \left[-i \sqrt{3} \xi_{1} X+\left(i \sqrt{3} \xi_{1}\right)^{-1} T\right]
\end{aligned}
$$

so that the respective determinant is

$$
\begin{aligned}
\operatorname{det} & M(X, T) \\
=\left[1+c_{1} \exp \left(-i \sqrt{3} \xi_{1} X+\left(i \sqrt{3} \xi_{1}\right)^{-1} T\right)\right]^{2}, & \\
c_{1} & =\frac{i \beta_{1}}{2 \sqrt{3} \xi_{1}} .
\end{aligned}
$$

As has been noted already, the singularity functions in form (242) with $M=1$ give rise to a single frequency for the continuous part of the spectral data. Hence, expression (265), having been substituted into concise formula (263), must provide us with the one-mode solution.

The condition that $W_{X}$ is real requires a restriction on the constant $\beta_{1}$ (if the constant $\xi_{1}$ is arbitrary but real). We have succeeded in obtaining this restriction (see Appendix A), namely, that the constant $c_{1}$, which in general is complexvalued one with $c_{1}=\left|c_{1}\right| \exp \left(i \chi_{1}\right)$, should possess unit modulus $\left|c_{1}\right|=1$, while the arbitrary real constant $\chi_{1}$ defines an initial shift of solution $X_{1}=\chi_{1} /\left(\sqrt{3} \xi_{1}\right)$ so that

$\operatorname{det} M(X, T)$

$$
=\left[1+\exp \left(-i \sqrt{3} \xi_{1}\left(X-X_{1}\right)+\frac{T}{i \sqrt{3} \xi_{1}}\right)\right]^{2} .
$$

The final result for one mode of the continuous spectrum is solution (263) with (266); namely,

$W(X, T)$

$$
\begin{aligned}
= & -3 \sqrt{3} \xi_{1} \tan \left(\frac{\sqrt{3}}{2} \xi_{1}\left(X-X_{1}\right)+\frac{T}{2 \sqrt{3} \xi_{1}}\right) \\
& + \text { const. }
\end{aligned}
$$

The corresponding solution for $U=W_{X}$ was obtained recently by other methods, for example, by the sine-cosine method [87], the $\left(G^{\prime} / G\right)$-expansion method [40], and the extended tanh-function method $[87,88]$. However, only the 
approach developed here and the solution in form (252) and (253) enable us to study the interaction of solitons and periodic waves.

We obtain periodic solutions even for $M=1$. Let us call attention once again to condition (209) which in the final result is shown below to restrict the region of $X$ for periodic solutions. At first glance it would seem that there is a contradiction between condition (209) and the periodic solution. Indeed, on the one hand, condition (209) demands that the solution $W(X, T)$ should vanish as $X \rightarrow-\infty$; on the other hand, the periodic solution obtained here does not satisfy condition (209). Nevertheless, consideration of the details enables us to find a reasonable explanation. So, in [75] at the derivation of relation (220) (see also (4.5) in [75]), the integral in (220) appears as a result of the integration on two sides of the boundaries between regular regions. For an understanding of this fact, relationship (252) from [75] plays an important role. Hence, the integration in (220) (also as in (4.5) in [75]) should be carried out over the lines $\omega_{2}(\xi+i \varepsilon)$ and $-\omega_{3}(\xi+i \varepsilon)$ as $\xi$ sweeps from $-\infty$ to $\infty$, where $\varepsilon>0$. As a result, in relationship (266) we should exchange $\xi_{1}$ for $\left(\xi_{1}+i \varepsilon\right)$ and that enables us to define the solution in the form

$$
\begin{aligned}
& W(X, T)=-i 6 \sqrt{3}\left(\xi_{1}+i \varepsilon\right) \\
& \cdot \frac{\exp (\sqrt{3} \varepsilon X) \exp \left(-i \sqrt{3} \xi_{1}\left(X-X_{1}\right)+T / i \sqrt{3} \xi_{1}\right)}{1+\exp (\sqrt{3} \varepsilon X) \exp \left(-i \sqrt{3} \xi_{1}\left(X-X_{1}\right)+T / i \sqrt{3} \xi_{1}\right)},
\end{aligned}
$$

which tends to constants as $|X| \rightarrow \infty$ at arbitrary $\varepsilon>$ 0 . Thus, on one hand, condition (209) is satisfied, and, on the other hand, at small $\varepsilon>0$ we have a sufficiently large region over $X$ where the solution associated with a finite $\varepsilon>0$ and the periodic solution associated with $\varepsilon=0$ are sufficiently close to each other. The region of $X$ with periodic solutions can be extended to sufficiently large, but finite, $|X|$. For any sequence $\varepsilon_{n} \rightarrow 0$ we remain within the inverse scattering theory [75] where condition (209) is not violated. Consequently, the periodic solution obtained at $\varepsilon=0$ is to be interpreted as the solution of the VPE which is valid on arbitrary but finite $|X|$.

11.1.2. The Two-Mode Solution. Let us consider a two-mode solution of the VPE. In this case $M(X, T)$ is a $4 \times 4$ matrix. For its determinant, according to (257) we find

$$
\begin{aligned}
\sqrt{\operatorname{det} M(X, T)} & =F(X, T) \\
& =1+c_{1} q_{1}+c_{2} q_{2}+b_{12} c_{1} c_{2} q_{1} q_{2},
\end{aligned}
$$

where $q_{i}, c_{i}$, and $b_{12}$ are defined by (261).

Since the solution $W_{X}$ should be real and the constants $\xi_{i}$ are arbitrary, but real, there are restrictions on the constants $c_{i}=\left|c_{i}\right| \exp \left(i \chi_{i}\right)$. The real constants $\chi_{i}$ define the initial shifts of solutions $X_{i}=\chi_{i} /\left(\sqrt{3} \xi_{i}\right)$. The analysis in considerable detail shows (see Appendix A) that the relations $\left|c_{1}\right|=\left|c_{2}\right|=$ $1 / \sqrt{b_{12}}$ are the sufficient conditions in order that $W_{X}$ be real. Thus, the interaction of two periodic waves for the VPE is described by relationship (263) with

$$
F(X, T)=1+\frac{1}{\sqrt{b_{12}}} q_{1}+\frac{1}{\sqrt{b_{12}}} q_{2}+q_{1} q_{2},
$$

where $b_{12}$ is as in (261), and the dependencies in $q_{i}$ now contain the phase shifts $X_{i}=\chi_{i} /\left(\sqrt{3} \xi_{i}\right)$ as follows:

$$
q_{i}=\exp \left(-i \sqrt{3} \xi_{i}\left(X-X_{i}\right)+\left(i \sqrt{3} \xi_{i}\right)^{-1} T\right)
$$

11.1.3. The Three-Mode Solution. For $N=0$ and $M=3$, in the relationship

$$
\begin{aligned}
F(X, T)= & 1+c_{1} q_{1}+c_{2} q_{2}+c_{3} q_{3}+c_{1} c_{2} b_{12} q_{1} q_{2} \\
& +c_{1} c_{3} b_{13} q_{1} q_{3}+c_{2} c_{3} b_{23} q_{2} q_{3} \\
& +c_{1} c_{2} c_{3} b_{12} b_{13} b_{23} q_{1} q_{2} q_{3}
\end{aligned}
$$

obtained from (253) (see also (258)) with $q_{i}, c_{i}$, and $b_{i j}$ as in (261), we write $c_{i}=\left|c_{i}\right| \exp \left(i \chi_{i}\right)$. Then the arguments $\chi_{i}$ determine the initial phase shifts of modes $X_{i}=\chi_{i} /\left(\sqrt{3} \xi_{i}\right)$. As is proved in Appendix A, the conditions on the constants $c_{i}$ are

$$
\begin{aligned}
& \left|c_{1}\right|=\frac{1}{\sqrt{b_{12} b_{13}}}, \\
& \left|c_{2}\right|=\frac{1}{\sqrt{b_{12} b_{23}}}, \\
& \left|c_{3}\right|=\frac{1}{\sqrt{b_{13} b_{23}}} .
\end{aligned}
$$

Hence the three-mode solution is relation (263) with

$$
\begin{aligned}
F(X, T)= & 1+\frac{1}{\sqrt{b_{12} b_{13}}}\left(q_{1}+q_{2} q_{3}\right) \\
& +\frac{1}{\sqrt{b_{12} b_{23}}}\left(q_{2}+q_{1} q_{3}\right) \\
& +\frac{1}{\sqrt{b_{13} b_{23}}}\left(q_{3}+q_{1} q_{2}\right)+q_{1} q_{2} q_{3} .
\end{aligned}
$$

Here the phase shifts $X_{i}$ are taken into account in $q_{i}$ by way of (271).

11.1.4. The Four-Mode Solution. For $N=0$ and $M=4$, the restrictions have the form (see Appendix A)

$$
\left|c_{i}\right|=\prod_{\substack{j=1 \\ j \neq i}}^{4} b_{i j}^{-1 / 2}, \quad 0 \leq b_{i j}=b_{j i} \leq 1, \quad i=1,2,3,4 .
$$


The function $F$ for real solution (263) is

$$
\begin{aligned}
F(X, T)= & +\frac{1}{\sqrt{b_{12} b_{13} b_{14}}}\left(q_{1}+q_{2} q_{3} q_{4}\right) \\
& +\frac{1}{\sqrt{b_{12} b_{23} b_{24}}}\left(q_{2}+q_{1} q_{3} q_{4}\right) \\
& +\frac{1}{\sqrt{b_{13} b_{23} b_{34}}}\left(q_{3}+q_{1} q_{2} q_{4}\right) \\
& +\frac{1}{\sqrt{b_{14} b_{24} b_{34}}}\left(q_{4}+q_{1} q_{2} q_{3}\right) \\
& +\frac{1}{\sqrt{b_{13} b_{14} b_{23} b_{24}}}\left(q_{1} q_{2}+q_{3} q_{4}\right) \\
& +\frac{1}{\sqrt{b_{12} b_{14} b_{23} b_{34}}}\left(q_{1} q_{3}+q_{2} q_{4}\right) \\
& +\frac{1}{\sqrt{b_{12} b_{13} b_{24} b_{34}}}\left(q_{1} q_{4}+q_{2} q_{3}\right) \\
& +q_{1} q_{2} q_{3} q_{4} .
\end{aligned}
$$

As before, $b_{i j}$ and $q_{i}$ are defined by (261) and (271), respectively.

11.2. The Solutions Associated with Bound State Spectrum. The features of the solutions associated with the bound state spectrum can be shown by considering the two-soliton solution for which $N=2$ and $M=0$. Solution (263) can be obtained through (257) with (260), that is,

$$
F(X, T)=1+c_{1} q_{1}+c_{2} q_{2}+b_{12} c_{1} c_{2} q_{1} q_{2}
$$

with

$$
\begin{gathered}
q_{i}=\exp \left(2 \theta_{i}\right) \\
2 \theta_{i}=\sqrt{3} \xi_{i} X-\left(\sqrt{3} \xi_{i}\right)^{-1} T, c_{i}=\frac{\beta_{i}}{2 \sqrt{3} \xi_{i}}, \\
b_{i j}=\left(\frac{\xi_{i}-\xi_{j}}{\xi_{i}+\xi_{j}}\right)^{2} \frac{\xi_{i}^{2}+\xi_{j}^{2}-\xi_{i} \xi_{j}}{\xi_{i}^{2}+\xi_{j}^{2}+\xi_{i} \xi_{j}}, \quad b_{i j} \geq 0 .
\end{gathered}
$$

In Appendix B it is proved that the constants $c_{i}$ have to be real. Moreover, the signs of $\alpha_{i}=c_{i} /\left|c_{i}\right|$ can independently take the values \pm 1 ; that is, we have four variants, namely, $\alpha_{1}=\alpha_{2}=1$, $\alpha_{1}=\alpha_{2}=-1, \alpha_{1}=-\alpha_{2}=1$, and $\alpha_{1}=-\alpha_{2}=-1$. Note that in [58] only the first two variants are discussed. The standard soliton solution for which $\alpha_{1}=\alpha_{2}=1$ and the singular soliton solutions for which $\alpha_{1}=\alpha_{2}=-1, \alpha_{1}=-\alpha_{2}=1$, and $\alpha_{1}=-\alpha_{2}=-1$ are obtained by means of relation (263)

$$
\begin{aligned}
U(X, T) & =W(X, T)_{X} \\
& =6 \frac{\partial^{2}}{\partial X^{2}} \ln (F)+6 \frac{\partial^{2}}{\partial X^{2}} \ln \left(G_{i}\right),
\end{aligned}
$$

Forms (B.3) and (B.7)-(B.10) for $F$ are more preferable, since we see that the solution is dependent on two combinations of the spectral parameters $\xi_{1}+\xi_{2}$ and $\xi_{1}-\xi_{2}$, but not three values $\xi_{1}, \xi_{2}$, and $\xi_{1}+\xi_{2}$ as it may appear from relation (279).

For $N \geq 3$ we give the conditions without proof. All the constants $c_{i}$ are to be real and the signs of $\alpha_{i}=c_{i} /\left|c_{i}\right|$ can be equal to \pm 1 independently of each other.

11.3. Real Soliton and Multimode Solutions of the VPE. In this subsection we will consider the general case, when both the bound state spectrum and the continuous spectrum are taken into account in the associated spectral problem. We will find the conditions on $c_{i}$ for real solutions of the VPE. To obtain the solution, we need to know the function $F$ (see (256)(259)).

Let the indexes $i$ and $i^{\prime}$ be related to the values involved in the bound state spectrum for which $\left(i, i^{\prime}\right) \leq N$, while the indexes $j$ and $j^{\prime}$ are related to the values involved in the continuous part of the spectral data for which $N<\left(j, j^{\prime}\right) \leq$ $N+M$

11.3.1. The Interaction of a Soliton with One-Mode Wave. The interaction of a standard soliton with periodic one-mode wave can be described by means of relations (257) with $N=1$ and $M=1$

$$
F(X, T)=1+c_{1} q_{1}+c_{2} q_{2}+b_{12} c_{1} c_{2} q_{1} q_{2}
$$

with $q_{i}$ and $b_{12}$ as in (262); namely,

$$
\begin{aligned}
& q_{1}=\exp \left(\sqrt{3} \xi_{1} X-\left(\sqrt{3} \xi_{1}\right)^{-1} T\right), \quad c_{1}=\frac{\beta_{1}}{2 \sqrt{3} \xi_{1}}, \\
& q_{2}=\exp \left(-i \sqrt{3} \xi_{2} X+\left(i \sqrt{3} \xi_{2}\right)^{-1} T\right), \\
& c_{2}=\frac{i \beta_{2}}{2 \sqrt{3} \xi_{2}} \\
& b_{12}=\left(\frac{\xi_{1}+i \xi_{2}}{\xi_{1}-i \xi_{2}}\right)^{2} \frac{\xi_{1}^{2}-\xi_{2}^{2}+i \xi_{1} \xi_{2}}{\xi_{1}^{2}-\xi_{2}^{2}-i \xi_{1} \xi_{2}},\left|b_{12}\right| \equiv 1
\end{aligned}
$$

First, we emphasize that the soliton one-mode wave (267) propagates in opposite directions. The soliton propagates in the positive direction of the $x$-axis, while one-mode wave (267) propagates in the negative direction of the $x$-axis.

Here we restrict ourselves to the simplest case $b_{12} c_{1} c_{2}=$ 1 that describes the interaction of a standard soliton with a one-mode wave. As follows immediately from Appendix C, for real solutions (263),

$$
W(X, T)=6 \frac{\partial}{\partial X} \ln (F(X, T))+\text { const. }
$$

where $F(X, T)$ is

$$
F(X, T)=1+\frac{1}{\sqrt{b_{12}}} q_{1}+\frac{1}{\sqrt{b_{12}}} q_{2}+q_{1} q_{2} .
$$

where $G_{i}$ are defined by (B.7)-(B.10). 
There is an exceptional case at $\xi_{1}=\xi_{2}$. Then we have $b_{12}=$ 1 , and $F=\left(1+q_{1}\right)\left(1+q_{2}\right)$. Consequently, solution (263) is reduced to the relation

$$
\begin{aligned}
W= & W_{1}+W_{2} \\
= & 3 \sqrt{3} \xi_{1} \tanh \left(\frac{\sqrt{3}}{2} \xi_{1}\left(X-X_{1}\right)-\frac{T}{2 \sqrt{3} \xi_{1}}\right) \\
& -3 \sqrt{3} \xi_{1} \tan \left(\frac{\sqrt{3}}{2} \xi_{1}\left(X-X_{0}\right)+\frac{T}{2 \sqrt{3} \xi_{1}}\right) \\
& + \text { const. }
\end{aligned}
$$

Here $W_{1}$ is the one-soliton solution and $W_{2}$ is solution (267) associated with one mode in the continuous part of the spectral data. The relationship $W=W_{1}+W_{2}$ is easily verified also by direct substitution into VPE (50). The two waves $W_{1}$ and $W_{2}$ propagate in different directions with the same speed without change of wave profile and phase shift. In other words, only in the case $\xi_{1}=\xi_{2}$ is there a simple superposition of the solutions $W_{1}$ and $W_{2}$. It is obvious that interactions of two solitons with a one-mode wave and/or of the twomode solution with one soliton do not satisfy this form of the interaction.

11.3.2. Real Solutions for $N$ Solitons and the M-Mode Wave. The interaction of $N$ solitons and $M$-mode wave (267) can be obtained by means of the function $F(X, T)$ with restrictions (C.8) given in Appendix C, namely,

$$
c_{i}=\frac{ \pm 1}{\sqrt{\prod_{j=1, j \neq i}^{N+M} b_{i j}}}, \quad b_{i j}=b_{j i}, \quad i=1, \ldots, N+M,
$$

and with the retention of the phase shifts $X_{i}$ in the quantities $q_{i}$ (C.2). The signs for $c_{i}$ in (285) can be chosen independently of each other. If the index $i$ in (285) is connected with the continuous part of the spectral data $(N<i \leq N+M)$, then the solutions generated by plus and minus signs in (285) are different only in the phase shifts. However, for the index $i$ from the bound state spectrum $(i \leq N)$, the solutions have different forms of function dependence. Here it is relevant to remember that there are standard soliton solutions and singular soliton solutions generated by different signs in the constants $c_{i}$ (285).

The solution will contain $(N+M)$ real constants $\xi_{i}$ for determining the values $b_{i j}$ and $(N+M)$ real constants $X_{i}$ to define the phase shifts.

In Sections 8-11 we have described the procedure for finding the solutions of the Vakhnenko-Parkes equation by means of the inverse scattering method. Both the bound state spectrum and the continuous spectrum are taken into account in the associated eigenvalue problem. The special form of the singularity functions enables us to obtain the multimode solutions. Sufficient conditions have been proved in order that the solutions become real functions. Finally we studied the interaction of solitons and the multimode wave.

In $[89,90]$ the Vakhnenko-Parkes equation has been generalized to an equation that is known as the generalized
Vakhnenko equation. It turns out that this new evolution equation possesses a wider variety of solutions, is integrable, and has been solved by both the Hirota method $[89,90]$ and the IST method $[47,91]$. Now this equation is investigated very actively in the scientific literature.

\section{Appendices}

\section{A. The Conditions on Constants $c_{i}$ for Multimode Waves}

In this appendix we will prove the conditions on the constants $c_{i}=\left|c_{i}\right| \exp \left(i \chi_{i}\right)$ for solutions associated with the continuous part of the spectral data only. We use the case $M=4$ as an example to prove the restrictions on the constants, at which the solution $W_{X}(X, T)$ is real. The auxiliary function $F(X, T)=\sqrt{\operatorname{det} M(X, T)}$ for finding the solution is (259); namely,

$$
\begin{aligned}
F(X, T)= & 1+c_{1} q_{1}+c_{2} q_{2}+c_{3} q_{3}+c_{4} q_{4} \\
& +c_{1} c_{2} b_{12} q_{1} q_{2}+c_{1} c_{3} b_{13} q_{1} q_{3}+c_{1} c_{4} b_{14} q_{1} q_{4} \\
& +c_{2} c_{3} b_{23} q_{2} q_{3}+c_{2} c_{4} b_{24} q_{2} q_{4}+c_{3} c_{4} b_{34} q_{3} q_{4} \\
& +c_{1} c_{2} c_{3} b_{12} b_{13} b_{23} q_{1} q_{2} q_{3} \\
& +c_{1} c_{2} c_{4} b_{12} b_{14} b_{24} q_{1} q_{2} q_{4} \\
& +c_{1} c_{3} c_{4} b_{13} b_{14} b_{34} q_{1} q_{3} q_{4} \\
& +c_{2} c_{3} c_{4} b_{23} b_{24} b_{34} q_{2} q_{3} q_{4} \\
& +c_{1} c_{2} c_{3} c_{4} b_{12} b_{13} b_{14} b_{23} b_{24} b_{34} q_{1} q_{2} q_{3} q_{4} .
\end{aligned}
$$

Here we redefine the values $c_{i}$ in such a way that $c_{i}=\left|c_{i}\right|$, since the arguments $\chi_{i}$ can always be introduced into the variables $q_{i}=\exp \left(i 2 \theta_{i}\right)$ with $2 \theta_{i}=-\sqrt{3} \xi_{i}\left(X-X_{i}\right)-\left(\sqrt{3} \xi_{i}\right)^{-1} T$ and $X_{i}=\chi_{i} /\left(\sqrt{3} \xi_{i}\right)$ serving as the shifts of solutions. The solution then has the form (263)

$$
W(X, T)=6 \frac{\partial}{\partial X} \ln (F(X, T))+\text { const. }
$$

The function $F$ is complex-valued; that is,

$$
\begin{aligned}
F=F_{\mathrm{Re}}+i F_{\mathrm{Im}}=|F| \exp \left(i \chi_{F}\right), \\
F_{\mathrm{Re}}=\operatorname{Re}(F), F_{\mathrm{Im}}=\operatorname{Im}(F), \tan \left(\chi_{F}\right)=\frac{F_{\mathrm{Im}}}{F_{\mathrm{Re}}} ;
\end{aligned}
$$

hence

$$
\frac{W(X, T)}{6}=\frac{\partial}{\partial X} \ln (|F|)+i \frac{\partial \chi_{F}}{\partial X}+\text { const. }
$$


If we succeed in making $\partial^{2} \chi_{F} / \partial X^{2} \equiv 0$ by the choice of the constants $c_{i}$, then the solution $W_{X}(X, T)$ will be a real function.

Let us write $F_{\mathrm{Im}}$ and $F_{\mathrm{Re}}$ in explicit forms; namely,

$$
\begin{aligned}
& F_{\mathrm{Im}}=c_{1} \sin \left(2 \theta_{1}\right)+c_{2} \sin \left(2 \theta_{2}\right)+c_{3} \sin \left(2 \theta_{3}\right)+c_{4} \\
& \cdot \sin \left(2 \theta_{4}\right)+c_{1} c_{2} b_{12} \sin \left[2\left(\theta_{1}+\theta_{2}\right)\right]+c_{1} c_{3} b_{13} \\
& \cdot \sin \left[2\left(\theta_{1}+\theta_{3}\right)\right]+c_{1} c_{4} b_{14} \sin \left[2\left(\theta_{1}+\theta_{4}\right)\right] \\
& +c_{2} c_{3} b_{23} \sin \left[2\left(\theta_{2}+\theta_{3}\right)\right]+c_{2} c_{4} b_{24} \\
& \cdot \sin \left[2\left(\theta_{2}+\theta_{4}\right)\right]+c_{3} c_{4} b_{34} \sin \left[2\left(\theta_{3}+\theta_{4}\right)\right] \\
& +c_{1} c_{2} c_{3} b_{12} b_{13} b_{23} \sin \left[2\left(\theta_{1}+\theta_{2}+\theta_{3}\right)\right] \\
& +c_{1} c_{2} c_{4} b_{12} b_{14} b_{24} \sin \left[2\left(\theta_{1}+\theta_{2}+\theta_{4}\right)\right] \\
& +c_{1} c_{3} c_{4} b_{13} b_{14} b_{34} \sin \left[2\left(\theta_{1}+\theta_{3}+\theta_{4}\right)\right] \\
& +c_{2} c_{3} c_{4} b_{23} b_{24} b_{34} \sin \left[2\left(\theta_{2}+\theta_{3}+\theta_{4}\right)\right] \\
& +c_{1} c_{2} c_{3} c_{4} b_{12} b_{13} b_{14} b_{23} b_{24} b_{34} \\
& \cdot \sin \left[2\left(\theta_{1}+\theta_{2}+\theta_{3}+\theta_{4}\right)\right], \\
& F_{\mathrm{Re}}=1+c_{1} \cos \left(2 \theta_{1}\right)+c_{2} \cos \left(2 \theta_{2}\right)+c_{3} \cos \left(2 \theta_{3}\right) \\
& +c_{4} \cos \left(2 \theta_{4}\right)+c_{1} c_{2} b_{12} \cos \left[2\left(\theta_{1}+\theta_{2}\right)\right]+c_{1} c_{3} b_{13} \\
& \cdot \cos \left[2\left(\theta_{1}+\theta_{3}\right)\right]+c_{1} c_{4} b_{14} \cos \left[2\left(\theta_{1}+\theta_{4}\right)\right] \\
& +c_{2} c_{3} b_{23} \cos \left[2\left(\theta_{2}+\theta_{3}\right)\right]+c_{2} c_{4} b_{24} \\
& \cdot \cos \left[2\left(\theta_{2}+\theta_{4}\right)\right]+c_{3} c_{4} b_{34} \cos \left[2\left(\theta_{3}+\theta_{4}\right)\right] \\
& +c_{1} c_{2} c_{3} b_{12} b_{13} b_{23} \cos \left[2\left(\theta_{1}+\theta_{2}+\theta_{3}\right)\right] \\
& +c_{1} c_{2} c_{4} b_{12} b_{14} b_{24} \cos \left[2\left(\theta_{1}+\theta_{2}+\theta_{4}\right)\right] \\
& +c_{1} c_{3} c_{4} b_{13} b_{14} b_{34} \cos \left[2\left(\theta_{1}+\theta_{3}+\theta_{4}\right)\right] \\
& +c_{2} c_{3} c_{4} b_{23} b_{24} b_{34} \cos \left[2\left(\theta_{2}+\theta_{3}+\theta_{4}\right)\right] \\
& +c_{1} c_{2} c_{3} c_{4} b_{12} b_{13} b_{14} b_{23} b_{24} b_{34} \\
& \cdot \cos \left[2\left(\theta_{1}+\theta_{2}+\theta_{3}+\theta_{4}\right)\right] \text {. }
\end{aligned}
$$

Let us try to present $F_{\mathrm{Im}}$ and $F_{\mathrm{Re}}$ in the forms

$$
\begin{aligned}
& F_{\mathrm{Im}}=2 G \sin \left(\theta_{1}+\theta_{2}+\theta_{3}+\theta_{4}\right), \\
& F_{\mathrm{Re}}=2 G \cos \left(\theta_{1}+\theta_{2}+\theta_{3}+\theta_{4}\right),
\end{aligned}
$$

where $G$ is the same in both formulas (A.6). This can be done if the following conditions are satisfied:

$$
\begin{aligned}
c_{1} & =c_{2} c_{3} c_{4} b_{23} b_{24} b_{34}, \\
c_{2} & =c_{1} c_{3} c_{4} b_{13} b_{14} b_{34}, \\
c_{3} & =c_{1} c_{2} c_{4} b_{12} b_{14} b_{24}, \\
c_{4} & =c_{1} c_{2} c_{3} b_{12} b_{13} b_{23}, \\
c_{1} c_{2} b_{12} & =c_{3} c_{4} b_{34}, \\
c_{1} c_{3} b_{13} & =c_{2} c_{4} b_{24}, \\
c_{1} c_{4} b_{14} & =c_{2} c_{3} b_{23}, \\
c_{1} c_{2} c_{3} c_{4} b_{12} b_{13} b_{14} b_{23} b_{24} b_{34} & =1 .
\end{aligned}
$$

It turns out that all these relations are valid when

$$
\begin{aligned}
& c_{1}=\frac{1}{\sqrt{b_{12} b_{13} b_{14}}}, \\
& c_{2}=\frac{1}{\sqrt{b_{12} b_{23} b_{24}}}, \\
& c_{3}=\frac{1}{\sqrt{b_{13} b_{23} b_{34}}}, \\
& c_{4}=\frac{1}{\sqrt{b_{14} b_{24} b_{34}}} .
\end{aligned}
$$

With conditions (A.8), the expression for $G$ reads as follows:

$$
\begin{aligned}
G= & \cos \left(\theta_{1}+\theta_{2}+\theta_{3}+\theta_{4}\right) \\
& +\frac{1}{\sqrt{b_{12} b_{13} b_{14}}} \cos \left(\theta_{1}-\theta_{2}-\theta_{3}-\theta_{4}\right) \\
& +\frac{1}{\sqrt{b_{12} b_{23} b_{24}}} \cos \left(\theta_{2}-\theta_{1}-\theta_{3}-\theta_{4}\right) \\
& +\frac{1}{\sqrt{b_{13} b_{23} b_{34}}} \cos \left(\theta_{3}-\theta_{1}-\theta_{2}-\theta_{4}\right) \\
& +\frac{1}{\sqrt{b_{14} b_{24} b_{34}}} \cos \left(\theta_{4}-\theta_{1}-\theta_{2}-\theta_{3}\right) \\
& +\frac{1}{\sqrt{b_{13} b_{14} b_{23} b_{24}}} \cos \left(\theta_{1}+\theta_{2}-\theta_{3}-\theta_{4}\right) \\
& +\frac{1}{\sqrt{b_{12} b_{14} b_{23} b_{34}}} \cos \left(\theta_{1}+\theta_{3}-\theta_{2}-\theta_{4}\right) \\
& +\frac{1}{\sqrt{b_{12} b_{13} b_{24} b_{34}}} \cos \left(\theta_{1}+\theta_{4}-\theta_{2}-\theta_{3}\right) .
\end{aligned}
$$

Now it is readily seen from (A.3) that

$$
\chi_{F}=\theta_{1}+\theta_{2}+\theta_{3}+\theta_{4}
$$

and as consequence we have

$$
\frac{\partial^{2} \chi_{F}}{\partial X^{2}}=\frac{\partial^{2} \chi_{F}}{\partial X \partial T}=0
$$


Hence, as follows from (A.4), the four-mode solution of the VPE can be reduced to real form with four real constants $X_{i}$ and four real constants $\xi_{i}$ (see (276)).

Without proof here we give the following conditions on the constants $c_{i}$ that ensure the real $M$-mode solution of the VPE:

$$
\left|c_{i}\right|=\prod_{\substack{j=1 \\ j \neq i}}^{M} b_{i j}^{-1 / 2}, \quad b_{i j}=b_{j i}, i=1, \ldots, M,
$$

where the $M$ constants $\xi_{i}$ determine the values $b_{i j}$ and the $M$ constants $X_{i}$ define the phase shifts for each mode. Note that relations (A.12) are sufficient conditions, but not necessary ones.

\section{B. The Conditions on Constants $c_{i}$ under the Interaction of Two Solitons}

Here we consider the conditions on signs for the constants $c_{i}$ under the interaction of two solitons $(N=2, M=0)$. We start with the relationship (257) and (260):

$$
F=1+c_{1} q_{1}+c_{2} q_{2}+b_{12} c_{1} c_{2} q_{1} q_{2} .
$$

Let us present the constants $c_{i}$ in the form

$$
\begin{aligned}
& c_{i}=\alpha_{i}\left|c_{i}\right| \exp \left(i \chi_{i}\right)=b_{12}^{-1 / 2} \exp \left(-\sqrt{3} \xi_{i} X_{i}+i \sigma_{i}\right), \\
& \sigma_{i}=\chi_{i}+\frac{\pi\left(1-\alpha_{i}\right)}{2} .
\end{aligned}
$$

All new constants $\chi_{i}$ and $X_{i}=-\ln \left(\left|c_{i} \sqrt{b_{12}}\right|\right) /\left(\sqrt{3} \xi_{i}\right)$ are real. We assume that $-\pi / 2<\chi_{i} \leq \pi / 2$; then the values $\alpha_{i}$ retain the signs of the constants $\operatorname{Re}\left(c_{i}\right)$; that is, $\alpha_{i}=\operatorname{Re}\left(c_{i}\right) /\left|\operatorname{Re}\left(c_{i}\right)\right|$. It is convenient for analyzing to rewrite (B.1) (the same as (257)) in the form

$$
F=2 \exp \left(\theta_{1}+\theta_{2}+\frac{i}{2}\left(\sigma_{1}+\sigma_{2}\right)\right) G
$$

with

$$
\begin{aligned}
G= & \cosh \left(\theta_{1}+\theta_{2}+\frac{i}{2}\left(\sigma_{1}+\sigma_{2}\right)\right) \\
& +b_{12}^{-1 / 2} \cosh \left(\theta_{1}-\theta_{2}+\frac{i}{2}\left(\sigma_{1}-\sigma_{2}\right)\right), \\
2 \theta_{i}= & \sqrt{3} \xi_{i}\left(X-X_{i}\right)-\left(\sqrt{3} \xi_{i}\right)^{-1} T .
\end{aligned}
$$

It is easily seen that only $G$ defines the solution, since $\left(\partial^{2} / \partial X^{2}\right) \ln (F)=\left(\partial^{2} / \partial X^{2}\right) \ln (G)$, while the conditions that the function $G$ is real are as follows:

$$
\begin{gathered}
\chi_{i}=0, \\
\sigma_{i}+\sigma_{2}=2 \pi k_{1}, \\
\sigma_{i}-\sigma_{2}=2 \pi k_{2}
\end{gathered}
$$

with $k_{i}=0,1$. Restrictions (B.6) lead to the requirements $\alpha_{1}=$ \pm 1 and $\alpha_{2}= \pm 1$, independently of each other, and $\chi_{i}=0$. Then the function $F$ has the following forms:

(1) For $\alpha_{1}=\alpha_{2}=1$

$$
\begin{aligned}
F & =2 \exp \left(\theta_{1}+\theta_{2}\right) G_{1}, \\
G_{1} & =\cosh \left(\theta_{1}+\theta_{2}\right)+b_{12}^{-1 / 2} \cosh \left(\theta_{1}-\theta_{2}\right) .
\end{aligned}
$$

(2) For $\alpha_{1}=\alpha_{2}=-1$

$$
\begin{aligned}
F & =2 \exp \left(\theta_{1}+\theta_{2}\right) G_{2}, \\
G_{2} & =\cosh \left(\theta_{1}+\theta_{2}\right)-b_{12}^{-1 / 2} \cosh \left(\theta_{1}-\theta_{2}\right) .
\end{aligned}
$$

(3) For $\alpha_{1}=-\alpha_{2}=1$

$$
\begin{aligned}
F & =2 \exp \left(\theta_{1}+\theta_{2}\right) G_{3}, \\
G_{3} & =-\sinh \left(\theta_{1}+\theta_{2}\right)+b_{12}^{-1 / 2} \sinh \left(\theta_{1}-\theta_{2}\right) .
\end{aligned}
$$

(4) For $\alpha_{1}=-\alpha_{2}=-1$

$$
\begin{aligned}
F & =2 \exp \left(\theta_{1}+\theta_{2}\right) G_{4}, \\
G_{4} & =-\sinh \left(\theta_{1}+\theta_{2}\right)-b_{12}^{-1 / 2} \sinh \left(\theta_{1}-\theta_{2}\right) .
\end{aligned}
$$

Hence, the standard soliton solution that follows from (B.7) and the singular soliton solutions that follow from (B.8)(B.10) are the real functions:

$$
U(X, T)=W_{X}(X, T)=6 \frac{\partial^{2}}{\partial X^{2}} \ln \left(G_{i}\right) .
$$

Now we rewrite the restrictions in a somewhat different form. By retaining the values of the phase shifts $X_{i}$ in the quantities $q_{i}$, we require

$$
\begin{aligned}
& c_{1}=\frac{ \pm 1}{\sqrt{b_{12}}}, \\
& c_{2}=\frac{ \pm 1}{\sqrt{b_{12}}},
\end{aligned}
$$

where the signs are independent of each other. Note that for this case there are two arbitrary real constants $\xi_{i}$ and two arbitrary real constants $X_{i}(i=1,2)$.

The notation in (B.7)-(B.10) shows that the solution is defined by two combinations of the spectral parameters, namely, $\xi_{1}+\xi_{2}$ and $\xi_{1}-\xi_{2}$, but not three values $\xi_{1}, \xi_{2}$, and $\xi_{1}+\xi_{2}$ as it may appear from (B.1).

The foregoing proof points to a way for finding the restrictions for any $N$ with $M=0$. Here it should be underlined that only at real $c_{i}$ with any sign of $\alpha_{i}=c_{i} /\left|c_{i}\right|$, are the soliton (or singular soliton) solutions determined by a real function. The conditions on the constants $c_{i}$ are as follows:

$$
c_{i}=\frac{ \pm 1}{\sqrt{\prod_{j=1, j \neq i}^{N} b_{12}}} \quad i=1, \ldots, N
$$

with the retention of the phase shifts $X_{i}$ in the quantities $q_{i}$. The signs for $c_{i}$ are independent of each other. The solution will contain the $N$ real constants $\xi_{i}$ for determining the values $b_{i j}$ and the $N$ real constants $X_{i}$ to define the phase shifts. 


\section{The Restrictions on Constants $c_{i}$ in the General Case}

In this appendix we will obtain the restrictions on the constants $c_{i}$ for real solutions, in the general case, taking into account the spectral data from both the bound state spectrum and the continuous spectrum. All features are inherent in the case $N+M=4$ considered here as an example. To find the solution by means of the inverse scattering method, one needs to know function (259):

$$
\begin{aligned}
F= & 1+c_{1} q_{1}+c_{2} q_{2}+c_{3} q_{3}+c_{4} q_{4}+b_{12} c_{1} c_{2} q_{1} q_{2} \\
& +b_{13} c_{1} c_{3} q_{1} q_{3}+b_{14} c_{1} c_{4} q_{1} q_{4}+b_{23} c_{2} c_{3} q_{2} q_{3} \\
& +b_{24} c_{2} c_{4} q_{2} q_{4}+b_{34} c_{3} c_{4} q_{3} q_{4} \\
& +b_{12} b_{13} b_{23} c_{1} c_{2} c_{3} q_{1} q_{2} q_{3}+b_{12} b_{14} b_{24} c_{1} c_{2} c_{4} q_{1} q_{2} q_{4} \\
& +b_{13} b_{14} b_{34} c_{1} c_{3} c_{4} q_{1} q_{3} q_{4}+b_{23} b_{24} b_{34} c_{2} c_{3} c_{4} q_{2} q_{3} q_{4} \\
& +b_{12} b_{13} b_{14} b_{23} b_{24} b_{34} c_{1} c_{2} c_{3} c_{4} q_{1} q_{2} q_{3} q_{4} .
\end{aligned}
$$

For convenience we rewrite the variables $q_{i}$ in the somewhat different form:

$$
\begin{aligned}
q_{i} & =\exp \left(2 \theta_{i}\right), \\
q_{j} & =\exp \left(i 2 \theta_{j}\right), \\
2 \theta_{i} & =\sqrt{3} \xi_{i}\left(X-X_{i}\right)-\left(\sqrt{3} \xi_{i}\right)^{-1} T, \\
2 \theta_{j} & =-\sqrt{3} \xi_{j}\left(X-X_{j}\right)-\left(\sqrt{3} \xi_{j}\right)^{-1} T .
\end{aligned}
$$

The phase shifts $X_{i}$ are arbitrary real constants. The values $b_{i j}$ in (C.1) are as in (262):

$$
\begin{aligned}
& b_{i i^{\prime}}=\left(\frac{\xi_{i}-\xi_{i^{\prime}}}{\xi_{i}+\xi_{i^{\prime}}}\right)^{2} \frac{\xi_{i}^{2}+\xi_{i^{\prime}}^{2}-\xi_{i} \xi_{i^{\prime}}}{\xi_{i}^{2}+\xi_{i^{\prime}}^{2}+\xi_{i} \xi_{i^{\prime}}}, \quad 0 \leq b_{i i^{\prime}} \leq 1, \\
& b_{j j^{\prime}}=\left(\frac{\xi_{j}-\xi_{j^{\prime}}}{\xi_{j}+\xi_{j^{\prime}}}\right)^{2} \frac{\xi_{j}^{2}+\xi_{j^{\prime}}^{2}-\xi_{j} \xi_{j^{\prime}}}{\xi_{j}^{2}+\xi_{j^{\prime}}^{2}+\xi_{j} \xi_{j^{\prime}}}, \quad 0 \leq b_{j j^{\prime}} \leq 1, \\
& b_{i j}=\left(\frac{\xi_{i}+i \xi_{j}}{\xi_{i}-i \xi_{j}}\right)^{2} \frac{\xi_{i}^{2}-\xi_{j}^{2}+i \xi_{i} \xi_{j}}{\xi_{i}^{2}-\xi_{j}^{2}-i \xi_{i} \xi_{j}}, \quad\left|b_{i j}\right| \equiv 1,
\end{aligned}
$$

where $\left(i, i^{\prime}\right) \leq N$ and $N<\left(j, j^{\prime}\right) \leq N+M$. Note that $b_{i i^{\prime}}$ and $b_{j j^{\prime}}$ are real values, and $b_{i j}^{*}=1 / b_{i j}$.

Without loss of generality, we will consider one set of values $N$ and $M$, for example, $N=1$ and $M=3$. Now we will show that restrictions (A.8)

$$
\begin{aligned}
& c_{1}=\frac{ \pm 1}{\sqrt{b_{12} b_{13} b_{14}}}, \\
& c_{2}=\frac{ \pm 1}{\sqrt{b_{12} b_{23} b_{24}}}, \\
& c_{3}=\frac{ \pm 1}{\sqrt{b_{13} b_{23} b_{34}}}, \\
& c_{4}=\frac{ \pm 1}{\sqrt{b_{14} b_{24} b_{34}}}
\end{aligned}
$$

(with $b_{i j}$ determined by (C.3)) are sufficient in order to obtain the real solutions.

For definiteness, we assume that $\sqrt{b_{i j}}$ is a root of the equation $x^{2}=b_{i j}$ with $-\pi / 2<\arg \sqrt{b_{i j}} \leq \pi / 2$. Let us rewrite relations (C.4) in the form $c_{i}=\alpha_{i} / \prod_{j=1, j \neq i}^{4} \sqrt{b_{i j}}$, where $\alpha_{i}=$ \pm 1 . It is evident that we can always attain $\alpha_{2}=\alpha_{3}=\alpha_{4}=1$ by choosing the phase shifts $X_{2}, X_{3}$, and $X_{4}$, while we need to consider the two cases $\alpha_{1}= \pm 1$. By defining $\sigma=\left(1-\alpha_{1}\right) / 2$, we can rewrite the auxiliary function $F$ from (C.1) in the form

$$
\begin{aligned}
F(X, T)= & 2 G e^{i \pi \sigma}\left(b_{12} b_{13} b_{14}\right)^{-1 / 4} \\
& \cdot \exp \left(\theta_{1}+\frac{i \pi \sigma}{2}+i \theta_{2}+i \theta_{3}+i \theta_{4}\right),
\end{aligned}
$$

$$
\begin{aligned}
G e^{i \pi \sigma} & =\left[\left(b_{12} b_{13} b_{14}\right)^{1 / 4}\right. \\
\cdot & \cos \left(-i \theta_{1}+\frac{\pi \sigma}{2}+\theta_{2}+\theta_{3}+\theta_{4}\right) \\
+ & \left(b_{12} b_{13} b_{14}\right)^{-1 / 4} \\
\cdot & \left.\cos \left(-i \theta_{1}+\frac{\pi \sigma}{2}-\theta_{2}-\theta_{3}-\theta_{4}\right)\right]+\left(b_{23} b_{24}\right)^{-1 / 2} \\
& \cdot\left[\left(\frac{b_{13} b_{14}}{b_{12}}\right)^{1 / 4} \cos \left(i \theta_{1}-\frac{\pi \sigma}{2}+\theta_{2}-\theta_{3}-\theta_{4}\right)\right. \\
+ & \left.\left(\frac{b_{13} b_{14}}{b_{12}}\right)^{-1 / 4} \cos \left(-i \theta_{1}+\frac{\pi \sigma}{2}+\theta_{2}-\theta_{3}-\theta_{4}\right)\right] \\
+ & \left(b_{23} b_{34}\right)^{-1 / 2}\left[\left(\frac{b_{12} b_{14}}{b_{13}}\right)^{1 / 4}\right. \\
+ & \left.\left(\frac{b_{12} b_{13}}{b_{14}}\right)^{-1 / 4} \cos \left(-i \theta_{1}+\frac{\pi \sigma}{2}+\theta_{4}-\theta_{2}-\theta_{3}\right)\right] \\
+ & \cos \left(i \theta_{1}-\frac{\pi \sigma}{2}+\theta_{3}-\theta_{2}-\theta_{4}\right)+\left(\frac{b_{12} b_{14}}{b_{13}}\right)^{-1 / 4} \\
+ & \left.\cos \left(-i \theta_{1}+\frac{\pi \sigma}{2}+\theta_{3}-\theta_{2}-\theta_{4}\right)\right]+\left(b_{24} b_{34}\right)^{-1 / 2}
\end{aligned}
$$

Since $b_{23}, b_{24}$, and $b_{34}$ are real, and $b_{1 j}^{*}=1 / b_{1 j}$ for $j=2,3,4$, it is evident that $G^{*}=G$; that is, the variable $G$ in the solution is a real-valued function. Hence the solution of the VPE, namely,

$$
\begin{aligned}
U(X, T) & =W_{X}(X, T)=6 \frac{\partial^{2}}{\partial X^{2}} \ln (F) \\
& =6 \frac{\partial^{2}}{\partial X^{2}} \ln (G),
\end{aligned}
$$

is a real quantity. 
Using this example, one can prove without difficulty that the procedure considered above can be extended to any $N$ and $M$ with restrictions (see also (A.12), (B.13), and (C.4)):

$$
c_{i}=\frac{ \pm 1}{\sqrt{\prod_{j=1, j \neq i}^{N+M} b_{i j}}}, \quad b_{i j}=b_{j i}, \quad i=1, \ldots, N+M,
$$

while the quantities $q_{i}$ retain the phase shifts $X_{i}$ (see (C.2)). The signs in (C.8) can be chosen independently of each other. For the interaction of $N$ solitons and the $M$-mode wave there are $(N+M)$ real constants $\xi_{i}$ and $(N+M)$ real constants $X_{i}$.

Note that restrictions (C.8) are sufficient conditions in order that the solution of the VPE is real.

\section{Conflict of Interests}

The authors declare that there is no conflict of interests regarding the publication of this paper.

\section{References}

[1] C. S. Gardner, J. M. Greene, M. D. Kruskal, and R. M. Miura, "Method for solving the Korteweg-deVries equation," Physical Review Letters, vol. 19, no. 19, pp. 1095-1097, 1967.

[2] J. F. Clarke, "Lectures on plane waves in reacting gases," Annales de Physique, vol. 9, no. 2, pp. 211-306, 1984.

[3] L. D. Landau and E. M. Lifshitz, Fluids Mechanics, Pergamon Press, New York, NY, USA, 1988.

[4] V. O. Vakhnenko, "High-frequency soliton-like waves in a relaxing medium," Journal of Mathematical Physics, vol. 40, no. 4, pp. 2011-2020, 1999.

[5] V. A. Vakhnenko, Periodic Short-Wave Perturbations in a Relaxing Medium, Institute of Geophysics, The Ukrainian Academy of Science, Kiev, Ukraine, 1991 (Russian).

[6] V. O. Vakhnenko, "The existence of loop-like solutions of a model evolution equation," Ukrainian Journal of Physics, vol. 42, no. 1, pp. 104-110, 1997 (Ukrainian).

[7] A. H. Nayfeh, Perturbation Methods, John Wiley \& Sons, New York, NY, USA, 1973.

[8] R. K. Dodd, J. C. Eilbeck, J. D. Gibbon, and H. C. Morris, Solitons and Nonlinear Wave Equations, Academic Press, London, UK, 1984.

[9] C. H. Su and C. S. Gardner, "Korteweg-de Vries equation and generalizations. III. Derivation of the Korteweg-de Vries equation and Burgers equation," Journal of Mathematical Physics, vol. 10, pp. 536-539, 1969.

[10] C. S. Gardner and G. K. Morikawa, "The effect of temperature on the width of a small-amplitude, solitary wave in a collisionfree plasma," Communications on Pure and Applied Mathematics, vol. 18, no. 1-2, pp. 35-49, 1965.

[11] H. Washimi and T. Taniuti, "Propagation of ion-acoustic solitary waves of small amplitude," Physical Review Letters, vol. 17, no. 19 , pp. 996-998, 1966.

[12] M. J. Ablowitz and H. Segur, Solitons and Inverse Scattering Transform, SIAM Press, Philadelphia, Pa, USA, 1981.

[13] S. P. Novikov, S. V. Manakov, L. P. Pitaevskii, and V. E. Zakharov, Theory of Solitons: The Inverse Scattering Method, Plenum Press, New York, NY, USA, 1984.

[14] R. Hirota, "Direct methods in soliton theory," in Solitons, R. K. Bullough and P. J. Caudrey, Eds., vol. 17 of Topics in Current Physics, pp. 157-176, Springer, Berlin, Germany, 1980.
[15] R. Hirota, The Direct Method in Soliton Theory, Cambridge University Press, Cambridge, UK, 2004.

[16] A. C. Newell, Solitons in Mathematics and Physics, SIAM, Philadelphia, Pa, USA, 1985.

[17] L. D. Faddeev and L. A. Takhtajan, Hamiltonian Methods in the Theory of Solitons, Springer, Berlin, Germany, 1987.

[18] A. M. Wazwaz, Partial Differential Equations and Solitary Waves Theory, Higher Education Press and Springer, 2009.

[19] G. B. Whitham, Linear and Nonlinear Waves, WileyInterscience, New York, NY, USA, 1974.

[20] V. A. Vakhnenko, "Solitons in a nonlinear model medium," Journal of Physics A: Mathematical and General, vol. 25, no. 15, pp. 4181-4187, 1992.

[21] E. J. Parkes, "The stability of solutions of Vakhnenko's equation," Journal of Physics. A. Mathematical and General, vol. 26, no. 22, pp. 6469-6475, 1993.

[22] L. A. Ostrovsky, "Nonlinear internal waves in a rotating ocean," Okeanologia, vol. 18, pp. 181-191, 1978.

[23] P. F. Byrd and M. D. Friedman, Handbook of Elliptic Integrals for Engineers and Scientists, Springer, Berlin, Germany, 1971.

[24] D. Takahashi and J. Satsuma, "Explicit solutions of magma equation," Journal of the Physical Society of Japan, vol. 57, no. 2, pp. 417-421, 1988

[25] E. Infeld and G. Rowlands, Nonlinear Waves, Solitons and Chaos, Cambridge University Press, Cambridge, UK, 1990.

[26] K. Murawski and R. G. Storer, "Stability of envelope waves," Wave Motion, vol. 11, no. 4, pp. 309-325, 1989.

[27] E. Infeld, J. Ziemkiewicz, and G. Rowlands, "The twodimensional stability of periodic and solitary wavetrains on a water surface over arbitrary depth Phys," Physics of Fluids, vol. 30, pp. 2330-2338, 1987.

[28] E. Infeld, "Self-focusing of nonlinear ion-acoustic waves and solitons in magnetized plasmas," Journal of Plasma Physics, vol. 33, no. 2, pp. 171-182, 1985.

[29] K. P. Das, F. W. Sluijter, and F. Verheest, "Stability of ion-acoustic cnoidal and solitary waves in a magnetized plasma," Physica Scripta, vol. 45, no. 4, pp. 358-363, 1992.

[30] K. Konno, Y. H. Ichikawa, and M. Wadati, "A loop soliton propagating along a stretched rope," Journal of the Physical Society of Japan, vol. 50, no. 3, pp. 1025-1026, 1981.

[31] H. Hasimoto, "A soliton on a vortex filament," Journal of Fluid Mechanics, vol. 51, no. 3, pp. 477-485, 1972.

[32] J. Lamb, "Solitons on moving space curves," Journal of Mathematical Physics, vol. 18, no. 8, pp. 1654-1661, 1977.

[33] J. M. Burgers, "A mathematical model illustrating the theory of turbulence," in Advances in Applied Mechanics, R. von Mises and T. von Kármán, Eds., pp. 171-199, Academic Press, New York, NY, USA, 1948.

[34] K. R. Rajagopal and L. Tao, Mechanics of Mixtures, World Scientific, Singapore, 1995.

[35] V. Struminskii, Mechanics and Technical Progress, Nauka Press, Moscow, Russia, 1980 (Russian).

[36] V. O. Vakhnenko and E. J. Parkes, "The two loop soliton solution of the Vakhnenko equation," Nonlinearity, vol. 11, no. 6, pp. 1457-1464, 1998.

[37] A. J. Morrison, E. J. Parkes, and V. O. Vakhnenko, "The N loop soliton solution of the Vakhnenko equation," Nonlinearity, vol. 12, no. 5, pp. 1427-1437, 1999.

[38] V. O. Vakhnenko, E. J. Parkes, and A. V. Michtchenko, "The Vakhnenko equation from the viewpoint of the inverse scattering method for the $\mathrm{KdV}$ equation," International Journal 
of Differential Equations and Applications, vol. 1, pp. 429-449, 2000.

[39] P. G. Estévez, "Reciprocal transformations for a spectral problem in 2+1 dimensions," Theoretical and Mathematical Physics, vol. 159, no. 3, pp. 763-769, 2009.

[40] R. Abazari, "Application of $\left(G^{\prime} / G\right)$-expansion method to travelling wave solutions of three nonlinear evolution equation," Computers \& Fluids, vol. 39, no. 10, pp. 1957-1963, 2010.

[41] F. Majida, H. Trikib, T. Hayatc, O. M. Aldossaryd, and A. Biswase, "Solitary wave solutions of the Vakhnenko-Parkes equation," Nonlinear Analysis: Modelling and Control, vol. 17, no. 1, pp. 60-66, 2012.

[42] Y. Ye, J. Song, S. Shen, and Y. Di, "New coherent structures of the Vakhnenko-Parkes equation," Results in Physics, vol. 2, pp. 170-174, 2012.

[43] V. O. Vakhnenko and E. J. Parkes, "The calculation of multisoliton solutions of the Vakhnenko equation by the inverse scattering method," Chaos, Solitons \& Fractals, vol. 13, no. 9, pp. 1819-1826, 2002.

[44] V. O. Vakhnenko and E. J. Parkes, "The singular solutions of a nonlinear evolution equation taking continuous part of the spectral data into account in inverse scattering method," Chaos, Solitons and Fractals, vol. 45, no. 6, pp. 846-852, 2012.

[45] V. O. Vakhnenko and E. J. Parkes, "Solutions associated with discrete and continuous spectrums in the inverse scattering method for the vakhnenko-parkes equation," Progress of Theoretical Physics, vol. 127, no. 4, pp. 593-613, 2012.

[46] V. O. Vakhnenko and E. J. Parkes, "Special singularity function for continuous part of the spectral data in the associated eigenvalue problem for nonlinear equations," Journal of Mathematical Physics, vol. 53, no. 6, Article ID 063504, 11 pages, 2012.

[47] V. O. Vakhnenko, E. J. Parkes, and A. J. Morrison, "A Bäcklund transformation and the inverse scattering transform method for the generalised Vakhnenko equation," Chaos, Solitons and Fractals, vol. 17, no. 4, pp. 683-692, 2003.

[48] N. J. Zabusky and M. D. Kruskal, "Interaction of 'solitons' in a collisionless plasma and the recurrence of initial states," Physical Review Letters, vol. 15, no. 6, pp. 240-243, 1965.

[49] R. Hirota and J. Satsuma, "A variety of nonlinear network equations generated from the Backlund transformation for the Toda lattice," Supplement of the Progress of Theoretical Physics, no. 59, pp. 64-100, 1976.

[50] R. M. Miura, Ed., Bäcklund Transformations, the Inverse Scattering Method, Solitons, and Their Applications, Springer, New York, NY, USA, 1976.

[51] R. Hirota and J. Satsuma, " $N$-soliton solutions of model equations for shallow water waves," Journal of the Physical Society of Japan, vol. 40, no. 2, pp. 611-612, 1976.

[52] M. Ito, "An extension of nonlinear evolution equations of the K-DV (mK-DV) type to higher orders," Journal of the Physical Society of Japan, vol. 49, no. 2, pp. 771-778, 1980.

[53] J. Hietarinta, "A search of bilinear equations passing Hirota's three-soliton condition: I. KdV-type bilinear equations," Journal of Mathematical Physics, vol. 28, no. 8, pp. 1732-1742, 1987.

[54] J. Hietarinta, "Hirota's bilinear method and its generalization," International Journal of Modern Physics A, vol. 12, no. 1, pp. 4351, 1997.

[55] B. Grammaticos, A. Ramani, and J. Hietarinta, "A search for integrable bilinear equations: the Painlevé approach "' Journal of Mathematical Physics, vol. 31, no. 11, pp. 2572-2578, 1990.
[56] M. Musette, F. Lambert, and J. C. Decuyper, "Soliton and antisoliton resonant interactions," Journal of Physics. A. Mathematical and General, vol. 20, no. 18, pp. 6223-6235, 1987.

[57] T. P. Moloney and P. F. Hodnett, "A new perspective on the $N$ soliton solution of the KdV equation," Proceedings of the Royal Irish Academy-Section A: Mathematical and Physical Sciences, vol. 89, no. 2, pp. 205-217, 1989.

[58] A.-M. Wazwaz, " $N$-soliton solutions for the Vakhnenko equation and its generalized forms," Physica Scripta, vol. 82, no. 6, Article ID 065006, 2010.

[59] P. F. Hodnett and T. P. Moloney, "On the structure during interaction of the two-soliton solution of the Korteweg-de Vries equation," SIAM Journal on Applied Mathematics, vol. 49, no. 4, pp. 1174-1187, 1989.

[60] P. G. Drazin and R. S. Johnson, Solitons: An Introduction, Cambridge University Press, 1989.

[61] V. O. Vakhnenko and E. J. Parkes, "A novel nonlinear evolution equation and its Backlund transformation," Reports of NAS of Ukraine, no. 6, pp. 91-96, 2001.

[62] V. O. Vakhnenko and E. J. Parkes, "A novel nonlinear evolution equation integrable by the inverse scattering method," Reports of NAS of Ukraine, no. 7, pp. 81-86, 2001.

[63] V. O. Vakhnenko and E. J. Parkes, "A novel nonlinear evolution equation integrable by the inverse scattering method," in Proceedings of the Fifth International Conference 'Symmetry in Nonlinear Mathematical Physics' (9-15 July, 2001, Kyiv), A. G. Nikitin, V. M. Boyko, and R. O. Popovych, Eds., vol. 43, part 1, pp. 384-391, 2002.

[64] V. A. Marchenko, "Eigenfunction expansions for non-selfadjoint singular differential operators of the second order," Transactions of the American Mathematical Society, vol. 25, pp. 77-130, 1960.

[65] H. Rund, "Variational problems and Bäcklund transformations associated with the sine Gordon and Korteweg-de Vries equations and their extensions," in Bäcklund Transformations, R. M. Miura, Ed., pp. 199-226, Springer, New York, NY, USA, 1974.

[66] R. Hirota, "A new form of Bäcklund transformations and its relation to the inverse scattering problem," Progress of Theoretical Physics, vol. 52, no. 5, pp. 1498-1512, 1974.

[67] J. Satsuma and D. J. Kaup, "A Bäcklund transformation for a higher order Korteweg-de Vries equation," Journal of the Physical Society of Japan, vol. 43, no. 2, pp. 692-697, 1977.

[68] J. Satsuma, "Higher conservation laws for the Korteweg-de Vries equation through Bäcklund transformation," Progress of Theoretical Physics, vol. 52, no. 4, pp. 1396-1397, 1974.

[69] M. Wadati, "The exact solution of the modified Korteweg-de vries equation," Journal of the Physical Society of Japan, vol. 32, no. 6, p. 1681, 1972.

[70] M. Wadati, “The modified Korteweg-de Vries equation," Journal of the Physical Society of Japan, vol. 34, pp. 1289-1296, 1973.

[71] M. J. Ablowitz, D. J. Kaup, A. C. Newell, and H. Segur, "Method for solving the sine-Gordon equation," Physical Review Letters, vol. 30, pp. 1262-1264, 1973.

[72] H. Flaschka, "On the Toda lattice. II. Inverse-scattering solution," Progress of Theoretical Physics, vol. 51, no. 3, pp. 703-716, 1974.

[73] P. D. Lax, "Integrals of nonlinear equations of evolution and solitary waves," Communications on Pure and Applied Mathematics, vol. 21, pp. 467-490, 1968.

[74] D. J. Kaup, "On the inverse scattering problem for cubic eigenvalue problems of the class $\psi_{x x x}+6 Q \psi_{x}+6 R_{\psi}=\lambda \psi$," Studies in Applied Mathematics, vol. 62, no. 3, pp. 189-216, 1980. 
[75] P. J. Caudrey, “The inverse problem for a general $N \times N$ spectral equation," Physica D, vol. 6, no. 1, pp. 51-66, 1982.

[76] P. J. Caudrey, "The inverse problem for the third order equation $u_{x x x}+q(x) u_{x}+r(x) u=-i \zeta^{3} u$," Physics Letters A, vol. 79, pp. 264-268, 1980 .

[77] V. E. Zakharov, "On stochastization of one-dimensional chains of nonlinear oscillators," Soviet Physics-JETP, vol. 38, pp. 108110, 1974.

[78] P. Deift, C. Tomei, and E. Trubowitz, "Inverse scattering and the Boussinesq equation," Communications on Pure and Applied Mathematics, vol. 35, no. 5, pp. 567-628, 1982.

[79] M. Musette and R. Conte, "Algorithmic method for deriving Lax pairs from the invariant Painlevé analysis of nonlinear partial differential equations," Journal of Mathematical Physics, vol. 32, no. 6, pp. 1450-1457, 1991.

[80] P. A. Clarkson and E. L. Mansfield, "Symmetry reductions and exact solutions of shallow water wave equations," Acta Applicandae Mathematicae, vol. 39, no. 1-3, pp. 245-276, 1995.

[81] A. N. W. Hone and J. P. Wang, "Prolongation algebras and Hamiltonian operators for Peakon equations," Inverse Problems, vol. 19, no. 1, pp. 129-145, 2003.

[82] V. O. Vakhnenko and E. J. Parkes, "Periodic and solitary-wave solutions of the Degasperis-Procesi equation," Chaos, Solitons \& Fractals, vol. 20, no. 5, pp. 1059-1073, 2004.

[83] V. O. Vakhnenko and E. J. Parkes, "The connection of the Degasperis-Procesi equation with the Vakhnenko equation," in Proceedinds of the Fifth International Conference 'Symmetry in Nonlinear Mathematical Physics', (23-29 June, 2003, Kyiv), A. G. Nikitin, V. M. Boyko, and R. O. Popovych, Eds., vol. 50, part 1, pp. 493-497, 2004.

[84] A. Boutet de Monvel and D. Shepelsky, "A Riemann-Hilbert approach for the Degasperis-Procesi equation," Nonlinearity, vol. 26, no. 7, pp. 2081-2107, 2013.

[85] A. B. de Monvel and D. Shepelsky, "The Ostrovsky-Vakhnenko equation by a Riemann-Hilbert approach," Journal of Physics A: Mathematical and Theoretical, vol. 48, no. 3, Article ID 035204, 2015.

[86] V. O. Vakhnenko, "Special form of the singularity function for continuous part of the spectral data in inverse scattering method," Ukrainian Journal of Physical, vol. 57, pp. 95-99, 2012.

[87] E. Yusufoğlu and A. Bekir, "The tanh and the sine-cosine methods for exact solutions of the MBBM and the Vakhnenko equations," Chaos, Solitons and Fractals, vol. 38, no. 4, pp. 11261133, 2008.

[88] E. J. Parkes, "Application of $(G / G)$-expansion method to travelling wave solutions of three nonlinear evolution equation" [Computers and Fluids 2010;39:1957-1963]," Computers \& Fluids, vol. 42, no. 1, pp. 108-109, 2011.

[89] A. J. Morrison and E. J. Parkes, "The $N$-soliton solution of a generalised Vakhnenko equation," Glasgow Mathematical Journal, vol. 43, pp. 65-90, 2001.

[90] A. J. Morrison and E. J. Parkes, “The $N$-soliton solution of the modified generalised Vakhnenko equation (a new nonlinear evolution equation)," Chaos, Solitons and Fractals, vol. 16, no. 1, pp. 13-26, 2003.

[91] V. O. Vakhnenko, E. J. Parkes, and A. J. Morrison, "Integrability of a novel nonlinear evolution equation," Reports of NAS of Ukraine, no. 6, pp. 86-92, 2002. 


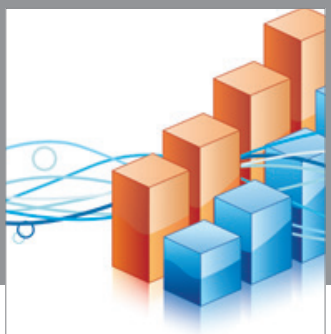

Advances in

Operations Research

vatem alat4

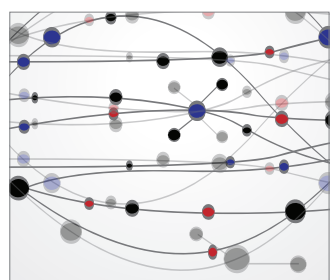

\section{The Scientific} World Journal
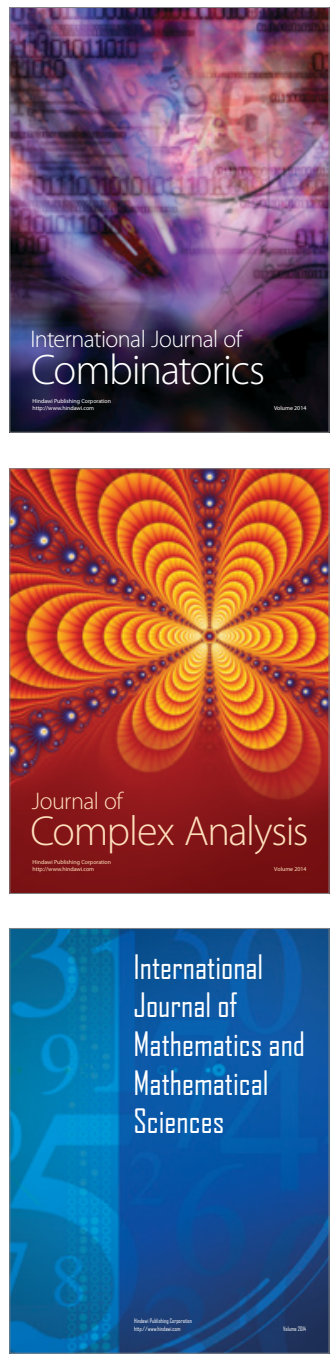
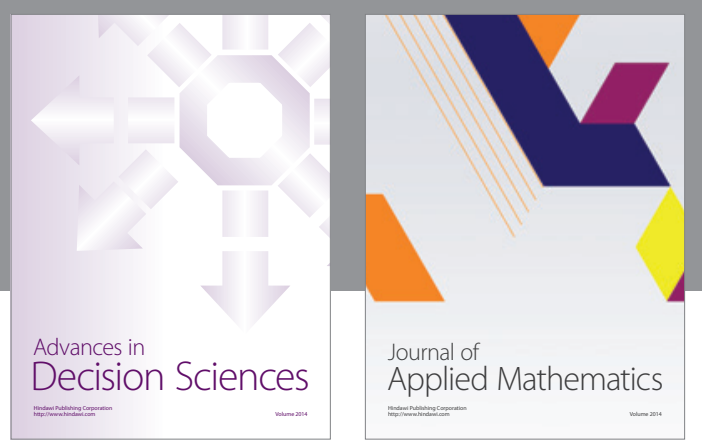

Algebra

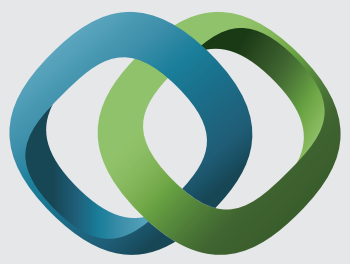

\section{Hindawi}

Submit your manuscripts at

http://www.hindawi.com
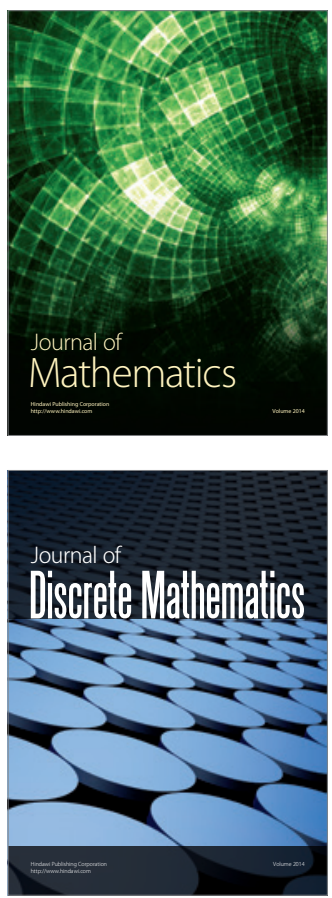

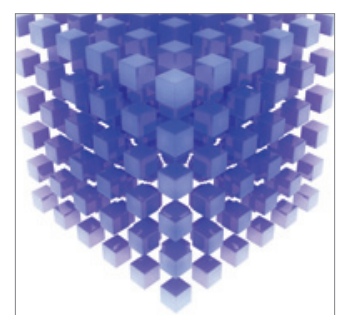

Mathematical Problems in Engineering
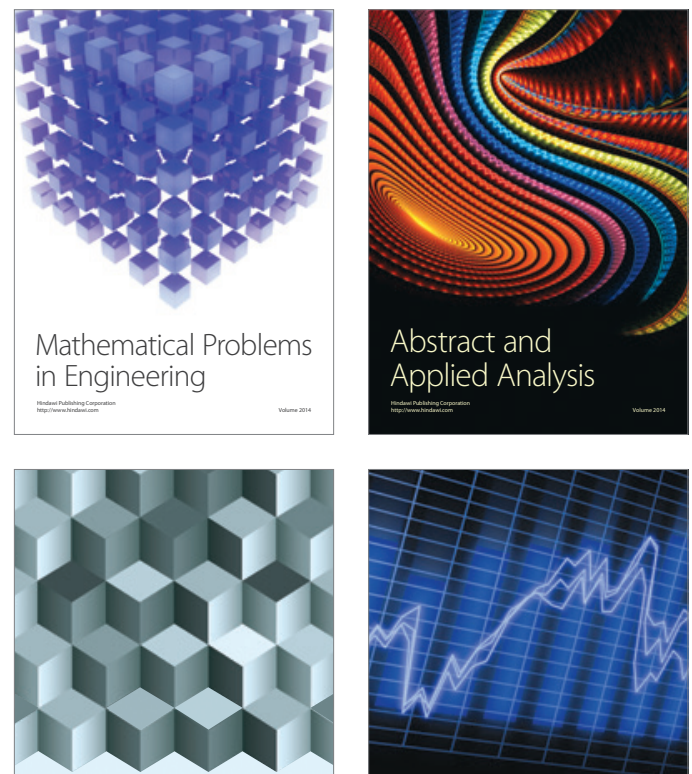

Journal of

Function Spaces

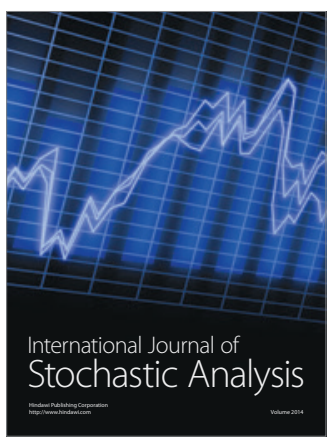

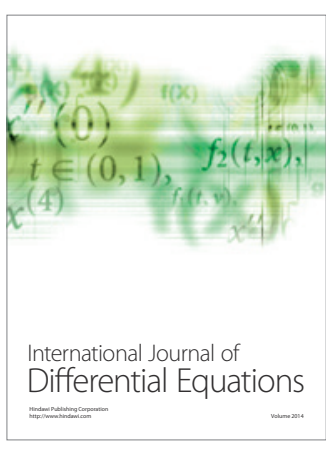
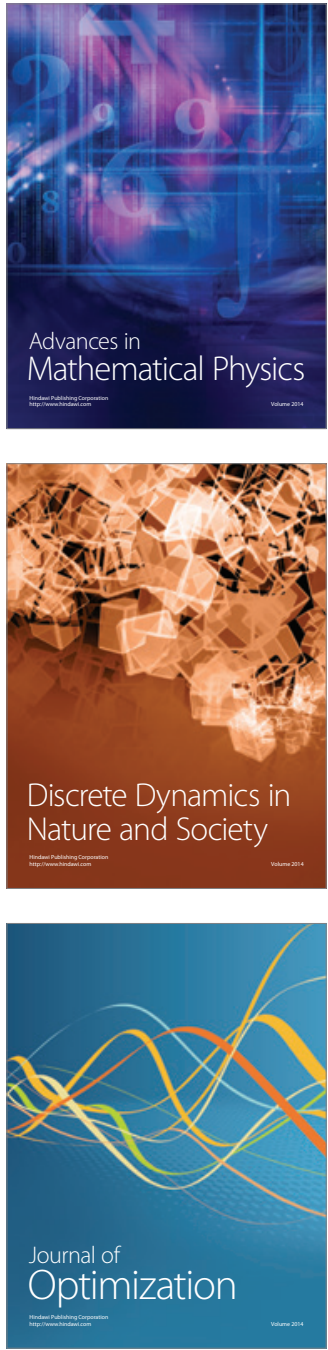